University of Rhode Island

DigitalCommons@URI

Open Access Master's Theses

2014

THE IMPACT OF HIGH AND LOW FODMAP CONDITIONS ON BLOOD GLUCOSE CONCENTRATIONS IN HEALTHY YOUNG ADULTS

Evan William Kerr

University of Rhode Island, evan_kerr@my.uri.edu

Follow this and additional works at: https://digitalcommons.uri.edu/theses

Recommended Citation

Kerr, Evan William, "THE IMPACT OF HIGH AND LOW FODMAP CONDITIONS ON BLOOD GLUCOSE CONCENTRATIONS IN HEALTHY YOUNG ADULTS" (2014). Open Access Master's Theses. Paper 469. https://digitalcommons.uri.edu/theses/469

This Thesis is brought to you for free and open access by DigitalCommons@URI. It has been accepted for inclusion in Open Access Master's Theses by an authorized administrator of DigitalCommons@URI. For more information, please contact digitalcommons-group@uri.edu. 
THE IMPACT OF HIGH AND LOW FODMAP CONDITIONS ON BLOOD GLUCOSE

CONCENTRATIONS IN HEALTHY YOUNG ADULTS

BY

EVAN WILLIAM KERR

A THESIS SUBMITTED IN PARTIAL FULFILLMENT OF THE

REQUIREMENTS FOR THE DEGREE OF

MASTERS OF SCIENCE

IN

NUTRITION

UNIVERSITY OF RHODE ISLAND

2014 
MASTER OF YOUR SCIENCE THESIS

OF

EVAN WILLIAM KERR

\section{APPROVED:}

Thesis Committee:

Major Professor Kathleen J. Melanson

Ingrid Lofgren

Matthew J. Delmonico

Nasser H. Zawia DEAN OF THE GRADUATE SCHOOL

UNIVERSITY OF RHODE ISLAND

2014 


\begin{abstract}
Objective: Fermentable oligosaccharides, disaccharides, monosaccharides and polyols (FODMAPs) are a group of carbohydrates that evade digestion in the small intestine and are subsequently metabolized by colonic microbiota. Colonic fermentation of FODMAPs has been linked to a prebiotic effect resulting in improved blood glucose response and subjective appetite. The purpose of this study was to examine how changes in FODMAP consumption effects blood glucose response and subjective appetite. Fasting breath hydrogen was also examined as an indicator of colonic fermentation.

Design: This study utilized a single blind, randomized, crossover design. Healthy participants $(\mathrm{n}=16)$ were instructed to follow a low-FODMAP and high-FODMAP diet for a period of three days separated by an 11-day "washout period." Fasting and post-prandial blood glucose were assessed via Cholestech. Subjective appetite was analyzed through use of visual analogue scales. Data were analyzed via repeated measures analysis of variance.
\end{abstract}

Results: Blood glucose concentrations did not vary significantly between the two dietary interventions $(\mathrm{p}=.111, \eta 2=.155)$. Reduced total area under the curve (TAUC) was seen after the high-FODMAP intervention, however, this decrease was not significant (6722 \pm 861 vs. $7149 \pm 1120 \mathrm{mg} / \mathrm{dl} *$ min, $\mathrm{p}=.178)$. No significant changes in subjective appetite were noted. Fasting breath hydrogen did not vary significantly between the two interventions, however, was found to be inversely correlated with glycemic response $(\mathrm{r}=-0.54, \mathrm{p}=.034)$. Outcomes were reanalyzed utilizing only subjects consuming greater than 4 grams of FODMAPs during high-FODMAP 
intervention. Reduced post-prandial blood glucose response $(\mathrm{F}(1,7 \mathrm{df})=7.21, \mathrm{p} .007)$ and blood glucose TAUC was seen $(\mathrm{t}=3.60, \mathrm{p}=.009)$ in this subset.

Conclusion: The High- FODMAP intervention resulted in a non-significant reduction in blood glucose. However, poor dietary compliance likely explains this result. The inverse relationship seen between breath hydrogen and blood glucose TAUC indicate a potential prebiotic effect. Individuals compliant with high FODMAP diet did show reduced glycemic response. Further research, with larger samples and longer interventions, is needed. 


\section{ACKNOWLEDGMENTS}

I have enjoyed my time at URI for the past two years. I have grown intellectually but also as a person and I have a lot of people to thank for that. I would like to first thank my advisor Dr. Melanson. I really enjoyed working in the Energy Balance Lab. Your enthusiasm for FODMAPs, HEBA and all things EBL made doing research a lot more enjoyable. I would also like to thank my committee members Dr. Lofgren and Dr. Delmonico for all the help that you have given me throughout the process of writing my thesis. Thank you Dr. Redding for being the chair of my defense.

I would also like to thank Val. Whether it was ordering supplies for my study or obtaining a travel stipend, you were always there to help guide me through the intricate network of paperwork, which we all know there is a form for everything at URI.

I would also like to thank the other graduate students. I truly enjoyed my time with you guys and thanks for putting up with me (especially Jim, Trish, Jess, Amy). I would also like to thank Carolyn for helping with data collection!

I would like to thank the Decorah eagles, for being so darn entertaining and for reminding me that there was an outdoors even though I was trapped indoors.

Last, but not least, I would like to thank my family. I have a truly great family. I would especially like to thank my Mom and Dad. This would never have been possible without your support, and I can never thank you enough. 


\section{PREFACE}

This manuscript was written to comply with the University of Rhode Island Graduate Manuscript Thesis Format. This thesis contains one manuscript entitled " The impact of High and Low- FODMAP Conditions on Blood Glucose Concentrations in Healthy Young Adults." This manuscript has been written in a form suitable for publication in The Journal of the Academy of Nutrition and Dietetics. 


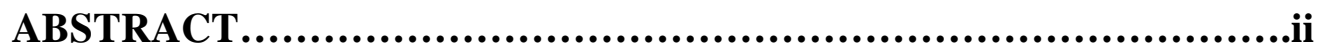

ACKNOWLEDGEMENTS...............................................iv

PREFACE ..............................................................

TABLE OF CONTENTS ..............................................vi

LIST OF TABLES .........................................................vii

LIST OF FIGURES......................................................viii

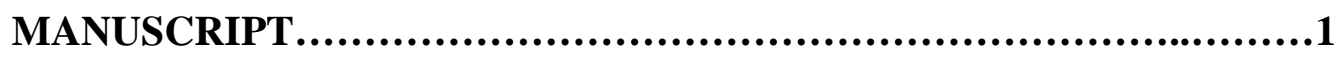

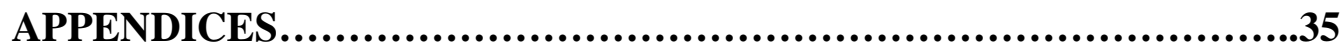

A. REVIEW OF LITERATURE....................................... 35

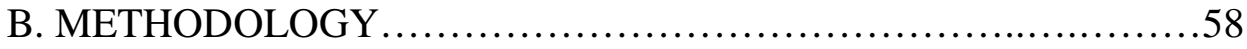

C. MACRONUTRIENT CONTENT OF TEST MEAL ...................66

D. BREATH HYDROGEN COLLECTION PROTOCOL...................... 67

E. IN LAB DATA COLLECTION MATERIALS........................68

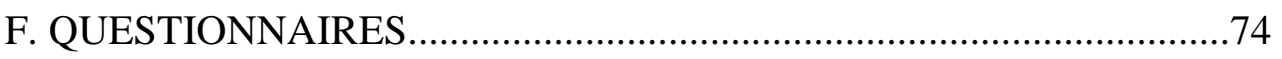

G. DIET INSTRUCTION BOOKLETS ................................77

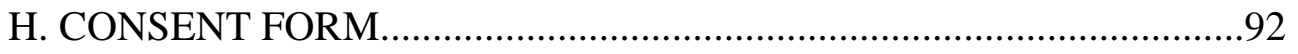

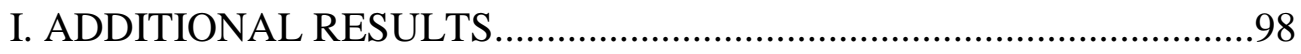

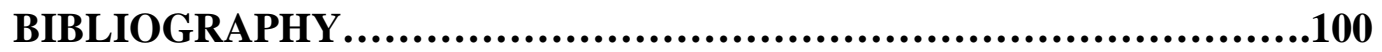




\section{Article I. LIST OF TABLES}

TABLE

PAGE

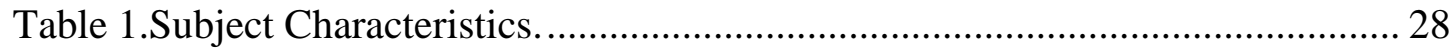

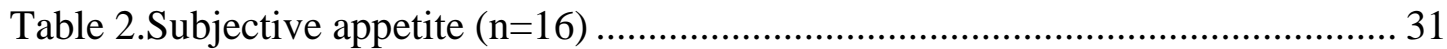

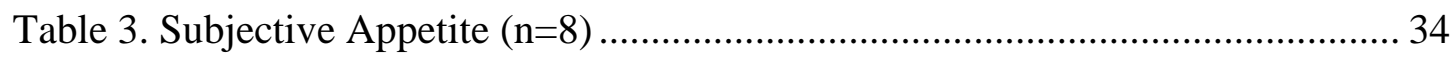




\section{LIST OF FIGURES}

FIGURE

PAGE

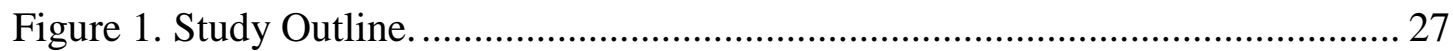

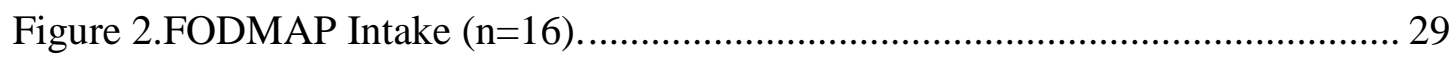

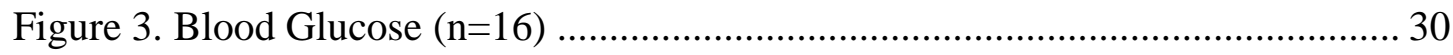

Figure 4. FODMAP Intake amongst subjects compliant with High-FODMAP diet .. 32

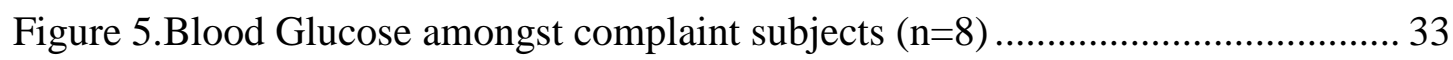




\section{MANUSCRIPT INTRODUCTION PAGE \\ MANUSCRIPT-1}

To be submitted to the Journal of the Academy of Nutrition and Dietetics

The impact of High and Low- FODMAP Conditions on Blood Glucose Concentrations in Healthy Young Adults

Evan Kerr, Ingrid Lofgren, Matthew Delmonico, Kathleen J. Melanson

Corresponding Author: Kathleen Melanson, PhD, RD, LDN

Department of Nutrition and Food Sciences

The University of Rhode Island

10 Ranger Rd, 202A Ranger Hall

Kingston, RI, 02881

Phone: 401.874 .4477

Email: kmelanson@uri.edu 


\title{
The impact of High and Low- FODMAP Conditions on Blood Glucose Concentrations in Healthy Young Adults
}

\author{
Evan Kerr, Matthew Delmonico, Ingrid Lofgren, Kathleen Melanson \\ Department of Nutrition and Food Sciences, University of Rhode Island \\ Kingston, Rhode Island 02881
}

\begin{abstract}
Objective: Fermentable oligosaccharides, disaccharides, monosaccharides and polyols (FODMAPs) are a group of carbohydrates that evade digestion in the small intestine and are subsequently metabolized by colonic microbiota. Colonic fermentation of FODMAPs has been linked to a prebiotic effect resulting in improved blood glucose response and subjective appetite. The purpose of this study was to examine how changes in FODMAP consumption effects blood glucose response and subjective appetite. Fasting breath hydrogen was also examined as an indicator of colonic fermentation.

Design: This study utilized a single blind, randomized, crossover design. Healthy participants $(\mathrm{n}=16)$ were instructed to follow a low-FODMAP and high-FODMAP diet for a period of three days separated by an 11-day "washout period." Fasting and post-prandial blood glucose were assessed via Cholestech. Subjective appetite was analyzed through use of visual analogue scales. Data were analyzed via repeated measures analysis of variance.
\end{abstract}

Results: Blood glucose concentrations did not vary significantly between the two dietary interventions $\left(\mathrm{p}=.111, \eta^{2}=.155\right)$. Reduced total area under the curve (TAUC) 
was seen after the high-FODMAP intervention, however, this decrease was not significant $(6722 \pm 861$ vs. $7149 \pm 1120 \mathrm{mg} / \mathrm{dl} * \mathrm{~min}, \mathrm{p}=.178)$. No significant changes in subjective appetite were noted. Fasting breath hydrogen did not vary significantly between the two interventions, however, was found to be inversely correlated with glycemic response $(\mathrm{r}=-0.54, \mathrm{p}=.034)$. Outcomes were reanalyzed utilizing only subjects consuming greater than 4 grams of FODMAPs during high-FODMAP intervention. Reduced post-prandial blood glucose response $(\mathrm{F}(1,7 \mathrm{df})=7.21, \mathrm{p} .007)$. and reduced blood glucose TAUC was also seen $(t=3.60, p=.009)$ in this subset.

Conclusion: The High- FODMAP intervention resulted in a non-significant reduction in blood glucose. However, poor dietary compliance likely explains this result. The inverse relationship seen between breath hydrogen and blood glucose TAUC indicate a potential prebiotic effect. Individuals compliant with high FODMAP diet did show reduced glycemic response. Further research, with larger samples and longer interventions, is needed. 


\section{Introduction:}

Fermentable oligosaccharides, disaccharides, monosaccharides, and polyols (FODMAPs) are a class of short-chained carbohydrates that are prevalent in the modern diet ${ }^{1,2}$. Common dietary FODMAPs include oligosaccharides such as fructans (or fructooligosacchrides) and galactans (or galactooligosaccharides), disaccharides such as lactose, monomers such as fructose, and polyols such as sorbitol and mannitol ${ }^{3}$. FODMAPs are associated with common functional properties including: limited absorption in the small intestine, high osmotic activity, and rapid fermentation by colonic microbiota ${ }^{4-6}$. Due to these functional properties, excess consumption of dietary FODMAPs has been linked to increased incidence of diarrhea, flatulence, as well as abdominal pain and distension amongst individuals with irritable bowel syndrome (IBS) ${ }^{2,7}$. Diets decreasing FODMAP intake have shown the ability to mitigate gastrointestinal symptoms and improve quality of life for individuals with IBS $^{8,9}$.

While reducing dietary FODMAPs has proven to be an effective means to manage IBS, long-term use of a low- FODMAP diet could have adverse health consequences and may not be appropriate for people without IBS. Low-FODMAP foods are generally considered to be high glycemic index (HGI) consisting of simple carbohydrates that are easily digested and absorbed ${ }^{10}$. Elevated intake of HGI foods have been found to increase risk of metabolic disease, diabetes, and cardiovascular disease ${ }^{10}$. Conversely, high-FODMAP foods are associated with a low glycemic index (LGI) ${ }^{10}$. These carbohydrates are not as readily absorbed by the small intestine. 
Thus LGI foods reduce postprandial glycemic response, which is important regarding development of chronic diseases such as diabetes and heart disease ${ }^{11}$.

In addition to reduced post-prandial glycemic response, LGI foods have demonstrated the ability to reduce glycemic response at subsequent meals ${ }^{12,13}$. This 'second meal effect' is likely due to a prebiotic effect attributed to increased colonic fermentation ${ }^{14-16}$. Malabsorbed carbohydrates, upon entering the colon, are rapidly metabolized, via fermentation reaction, by the resident colonic microbiota. Short-chain fatty acids (SCFA), byproducts of anaerobic metabolism, interact with colonic epithelial cells and have been linked to increased post-prandial expression of numerous gut peptide hormones, most notably glucagon like peptide -1 (GLP-1), which has been shown to have an important role regarding the improvement of glycemic response ${ }^{17,18}$. Increased colonic fermentation, as measured by breath hydrogen, is inversely related to blood glucose response ${ }^{19,20}$. Furthermore, colonic fermentation has been found to decrease post-prandial blood glucose response by as much as $15 \%$ amongst a healthy sample ${ }^{21}$. Increased colonic fermentation associated with consumption of FODMAP-dense foods indicates that FODMAPs have the potential to effect blood glucose response in a manner that is independent of glycemic index ${ }^{12}$. However, a possible prebiotic effect associated with FODMAP specific interventions has not been examined.

Increased colonic fermentation has also been linked to decreases in subjective appetite ratings. Supplementation of fructooligosaccharides has been found to promote expression of GLP-1, which leads to reduced ratings of subjective hunger in 
both clinical and free-living settings ${ }^{22,23}$. Furthermore, increased colonic

fermentation has been associated with decreased energy intake ${ }^{24}$.

Research, to this point, has indicated that increases in colonic fermentation of carbohydrates will reduce post-prandial blood glucose response. However, these studies involved test meals or supplementation designed to deliver a targeted amount of oligosaccharides or soluble fibers. The ability of a FODMAP specific dietary intervention to elicit this effect has not been examined. Furthermore, a possible prebiotic effect associated with increased FODMAP intake, in a free-living setting, has not been examined. The present study examined how implementation of low- and high- FODMAP diets, in a free- living setting, would impact fasting and post-prandial glycemic response amongst healthy participants. Additionally, this study examined how changes in dietary FODMAPs would affect subjective hunger, satiety and prospective consumption, in both fasting and post-prandial states. Total FODMAP intake and fasting breath hydrogen were analyzed as a means to determine dietary compliance.

\section{Materials and Methods:}

Study Design:

The study utilized a randomized, single-blinded, cross-over design that compared low and high-FODMAP diet-interventions in a free-living setting. Study design and laboratory procedures are outlined in figure 1 . This study consisted of five separate data collection days. Initial data collection (i.e. prior to any dietary intervention or outcome testing) consisted of screening, completion of informed consent, instruction regarding study protocol, anthropometrics, and body composition assessment (via BODPOD). Baseline data collection took place on a Tuesday and 
required that the subject arrive at the laboratory following a 10-hour fasting period. Fasting anthropometrics, blood glucose, breath hydrogen and subjective appetite ratings were obtained. A high FODMAP test meal (described below) was then administered and post-prandial blood glucose response and subjective appetite were measured at 30 and 60 min post consumption of the test meal. A 24-hour dietary recall was taken using the Nutritional Data System for Research (NDSR). At the conclusion of the 1-hour test period, individuals were randomly assigned to follow either diet 1 (low- FODMAP) or diet 2 (high- FODMAP) for a period of three days. Subjects reported to the laboratory for post-intervention testing on the following Friday. The post-intervention laboratory protocol was identical to that of baseline testing. After post-intervention testing, subjects undertook an 11-day washout period and the protocol, with the alternative diet, was repeated beginning on the following Tuesday. Order randomization started with a coin flip for the first subject, and subjects altered order thereafter.

\section{Subjects:}

All subjects participating in this study were recruited as a convenience sample from the student population of the University of Rhode Island. Exclusionary criteria included gastrointestinal disorders such as: celiac disease, IBS, lactose or gluten intolerance, diverticular disease, colitis such as Cohn's disease or ulcerative colitis and stomach ulcers. Additional exclusion criteria included currently following a weight loss diet, food allergies, smoking, pregnancy or lactation, diabetes, adrenal disease, kidney or bladder problems, a thyroid disease or currently taking any appetite suppressant medication. Subjects were advised to keep physical activity constant 
throughout the course of the study. The study was approved by the Institutional Review Board of the University of Rhode Island and subjects provided written informed consent prior to participating.

Blood Glucose Analysis:

Blood glucose was analyzed at three separate time points during each laboratory session. Capillary blood from subjects fingers was sampled at 0 minutes (fasting), 30-minutes post-test meal, and 60-minutes-post test meal. Protocols for obtaining capillary blood samples were obtained from Alere Inc (Waltham, Ma). At each time point, $40 \mu \mathrm{L}$ aliquots of capillary blood were obtained and subsequently analyzed via Cholestech LDX (Alere Inc., Waltham MA). Assessment of Subjective Hunger, Satiety and Prospective Consumption:

Subjective appetite was assessed using 10-cm visual analog scales (VAS). During laboratory sessions subjects completed VAS questionnaires regarding satiety, hunger, and prospective food consumption. VAS has been determined to be an effective method for assessing these outcomes ${ }^{25}$. Assessment of FODMAP Intake:

FODMAP intake was assessed through 24- hour recalls utilizing NDSR. Recalls were conducted using the NDSR software version 12 developed by the Nutrition Coordinating Center (NCC), University of Minnesota, Minneapolis. NDSR utilizes a multiple pass method described in full elsewhere ${ }^{26}$. All 4 dietary recalls reflected Mondays (pre-intervention) and Thursdays (post-intervention). Total intake of fructose, lactose, and sugar alcohols were compiled from NDSR output files for analysis. The extent to which dietary fructose and lactose are malabsorbed is highly 
variable. Fructose is absorbed efficiently when consumed in equimolar amounts as glucose ${ }^{27}$. A healthy individual can consume between 25-50 grams of lactose before malabosrption can occur and was excluded from analysis ${ }^{28,29}$. Thus total FODMAP intake was considered fructose consumed in excess of glucose, and sugar alcohols. Oligosaccharide intake is not assessed by NDSR databases and to our knowledge is not available in any nutrient database reflecting the United States food supply.

\section{Breath Hydrogen:}

Fasting Breath hydrogen was assessed at all baseline and post-intervention data collection days. Samples were collected with the QuinTron AveoSampler (QuinTron Instrument Co, WI, USA), as described elsewhere ${ }^{30}$. Once obtained, samples were immediately analyzed utilizing the QuinTron Microlyzer- CM (QuinTron Instrument Co, WI, USA). The instrument was calibrated 30 min prior to use, utilizing 98-PPM reference gas (QuinTron Instrument Co, WI, USA) ${ }^{31}$. Samples were collected in duplicate and the results were averaged.

\section{Anthropometrics:}

All anthropometric measurements were obtained at the beginning of each laboratory session, after the subject had undergone a 10-hour overnight fast and had voided bladder. Height, weight, and waist circumference were all measured and BMI was calculated. The protocol utilized for collection of anthropometric data has been described elsewhere ${ }^{32}$.

High- FODMAP Test Meal:

The test meal was designed to mimic free-living conditions. The $25 \mathrm{~g}$ of FODMAPS associated with the test meal have been found to induce increases in 
breath hydrogen concentration indicating carbohydrate malabsorption ${ }^{8}$. The test meal consisted of: 2 pieces of whole grain bread, 1 tsp. of sugar free preserves, 1 tsp. of honey, $40 \mathrm{~g}$ of raisins, and $12 \mathrm{oz}$. of milk. This meal consists of $478 \mathrm{kcal}$ and contained $25 \mathrm{~g}$ of FODMAPs per serving Implementation of Dietary Intervention:

At the conclusion of baseline data collection, each subject was provided with educational materials and one on one verbal instruction (approximately 15 minutes in duration) regarding the dietary intervention to which he or she was assigned. In order to ensure that the participants were unaware of the intervention to which they were assigned, the low- FODMAP and high- FODMAP interventions were referred to as "diet 1" and "diet 2" respectively. Subjects were provided with a booklet to assist them with adherence to the high or low FODMAP conditions to which they were assigned. Dietary booklets were made specifically for the purposes of this study utilizing previous research studies ${ }^{2,33-35}$. Outside of the laboratory setting no food of any kind was supplied to study participants.

\section{Power Calculations:}

Sample size calculations were performed based on differences in post-prandial blood glucose response seen in a previous study by Nilsson et al ${ }^{20}$. This study found that peak blood glucose concentrations decreased $1 \pm 0.3 \mathrm{mmol} / \mathrm{L}(18 \pm 5 \mathrm{mg} / \mathrm{dL})$ from control to experimental conditions. Calculations show an effect size of .333. All calculations were based on an alpha of 0.05 and power of 0.80 , and were completed using $\mathrm{G}^{*}$ Power (version 3.1.7). According to these calculations, a 8-person sample size would be adequate to achieve sufficient power. 
Statistics:

Statistical analyses were performed using SPSS, version 22.0 (IBM

Corporation, Summers, NY, USA). Analysis of skewness and kurtosis show that all outcomes met criteria for normality. Data were checked for outliers utilizing Grubbs test for outliers ${ }^{36}$. All outcomes were analyzed using repeated measures analysis of variance (treatment x time). Post hoc t-tests were utilized where appropriate. Partial eta squared $\left(\eta^{2}\right)$ was calculated to estimate effect size, with a small effect (.10), medium effect (.250), and large effect $(.40)^{37}$. Paired t-tests were utilized to examine within treatment interactions (baseline and intervention). Total area under the curve (TAUC) was calculated for blood glucose as well as subjective appetite. Pearson correlations were utilized to examine relationships between dietary FODMAP intake and subjective appetite as well as breath hydrogen and blood glucose. Significance was considered at $\mathrm{p}<.05$.

\section{$\underline{\text { Results: }}$}

A total of 20 participants were recruited and enrolled in the study. Four subjects withdrew from the study. One withdrew due to medical reasons unrelated to this study. Three subjects attended the initial screening visit, however, failed to complete the final four visits. Data obtained from the remaining 16 participants were utilized for analysis. Demographic information is presented in table 1. There were no significant differences regarding demographic variables of individuals assigned to the two orders. There was no evidence of an order effect regarding blood glucose at any time point as Treatment $*$ order $(\mathrm{F}(1,12 \mathrm{df})=.030, \mathrm{p}=.865)$, time $*$ order $(\mathrm{F}(1,12 \mathrm{df})=.120$, and treatment*time* $\operatorname{order}(\mathrm{F}(1,12 \mathrm{df})=.101, \mathrm{p}=.904)$ interactions were all non-significant 
Assessment of Dietary Compliance FODMAP Intake:

Figure 2 shows FODMAP intake during the two interventions. There was a significant treatment effect between the two dietary interventions $(F(1,15 \mathrm{df})=9.845$, $\left.\mathrm{p}=.007, \eta^{2}=0.395\right)$ as well as a significant treatment *time interaction $(\mathrm{F}(1,15 \mathrm{df})=5.316$, $\mathrm{p}=.033, \eta=0.262$ ). Within treatments analysis showed a significant decrease in FODMAP intake under the low- FODMAP condition when compared to baseline (2.26 \pm 3.00 grams to $0.52 \pm 0.55, \mathrm{t}=2.275, \mathrm{p}=.038)$. FODMAP intake during the highFODMAP intervention, increased, however, this increase was not significant (3.23 \pm $4.68(\mathrm{~g})$ to $5.04 \pm 5.36, \mathrm{t}=1.044, \mathrm{p}=.313)$. High-FODMAP diet resulted in a significant increase in dietary FODMAPs post-intervention, as compared to the lowFODMAP diet (5.04 \pm 5.36 vs. $0.52 \pm 0.55 \mathrm{~g}$ respectively; $\mathrm{t}=-3.513, \mathrm{p}=.003)$. Assessment of Dietary Compliance via Fasting Breath Hydrogen:

Data from one subject was determined to be an outlier and was excluded from analysis. High-FODMAP diet showed modest increases in breath hydrogen when compared to the low-FODMAP intervention ( $6.6 \pm 8.0$ vs. $2.7 \pm 3.3 \mathrm{ppm})$. There was a significant treatment effect $\left(\mathrm{F}(1,12)=6.282, \mathrm{p}=.028, \eta^{2}=.344\right)$ however, time and treatment* time trends were not significant (Time: $p=.763, \eta^{2}=.008$; treatment*time: $\mathrm{p}=.539, \eta^{2}=.032$ ). There were no within treatment effects noted (Low-FODMAP: $\mathrm{t}=1.426, \mathrm{p}=.179$, High- FODMAP: $\mathrm{t}=-.216, \mathrm{p}=.833)$.

\section{Blood Glucose:}

Two subjects were excluded from biochemical analysis due to abnormal blood glucose values. Figure 3 compares blood glucose response during the low- and highFODMAP conditions. Following the high- FODMAP intervention, there was a non- 
significant reduction in blood glucose response 30 minutes following consumption of the test meal $(124.64 \pm 22.84$ vs. $137.31 \pm 30.52 \mathrm{mg} / \mathrm{dL}, \mathrm{t}=1.585, \mathrm{p}=.137)$. Treatment and treatment* time interactions were also not significant, and had small effect sizes $\left(\mathrm{F}_{1,13 \mathrm{df}}\right)=1.631, \mathrm{p}=.224, \eta 2=0.111$ and $\left.\mathrm{F}_{(1,13 \mathrm{df}}\right)=2.391, \mathrm{p}=0.111, \eta^{2}=0.155$, respectively). Likewise, total AUC was modestly lower under the high-FODMAP condition $(6722 \pm 861$ vs. $7149 \pm 1120 \mathrm{mg} / \mathrm{dl} * \mathrm{~min})$, however, these differences were not statistically significant $(\mathrm{t}=1.424, \mathrm{p}=.178)$. Analysis with Pearson correlations showed that blood glucose response 30 minutes after the test meal were inversely correlated to fasting breath hydrogen levels during the high-FODMAP intervention $(\mathrm{r}=-.546$, $\mathrm{p}=$.043). Likewise, blood glucose TAUC, under the high-FODMAP condition was inversely correlated to fasting breath hydrogen $(\mathrm{r}=-.549, \mathrm{p}=.034)$.

\section{Subjective Appetite:}

Post-intervention analysis of subjective hunger showed no significant differences between the high and low-FODMAP diet. Treatment $(\mathrm{F}(1,15 \mathrm{df})=0.118$, $\mathrm{p}=.736)$ and treatment $*$ time $(\mathrm{F}(1,15 \mathrm{df})=0.383, \mathrm{p}=.685)$ trends were not significant. Under fasting conditions, subjective hunger, under the low-FODMAP intervention was significantly elevated over pre-intervention $(50.94 \pm 27.08$ vs $64.63 \pm 23.40(\mathrm{~mm})$, $\mathrm{t}=-2.554, \mathrm{p}=.022)$. Total area under the curve for subjective hunger is presented in table 2. No significant differences between subjective ratings of satiety were seen between the high and low-FODMAP interventions. Treatment $(\mathrm{F}(1,15 \mathrm{df})=.004, \mathrm{p}=.947$,

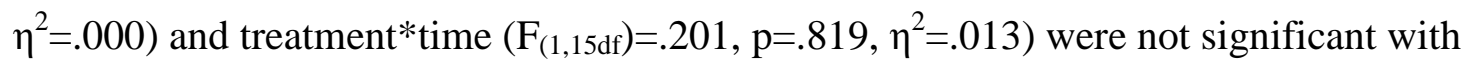
very small effect sizes. TAUC for subjective satiety was analyzed and no significant findings were noted. Comparison of post-intervention data regarding prospective 
consumption showed non-significant treatment $\left(\mathrm{F}(1,15 \mathrm{df})=996, \mathrm{p}=.334, \eta^{2}=.062\right)$ and treatment*time $\left(\mathrm{F}(1,15 \mathrm{df})=.043, \mathrm{p}=.958, \eta^{2}=.003\right)$ interactions. No significant withintreatment differences were seen regarding prospective consumption. Total area under the curve for prospective consumption showed no significant differences between the high and low-FODMAP diet (table 2).

\section{Data analysis with dietary compliance:}

Biochemical analysis was reassessed including individuals who were most compliant with the high-FODMAP dietary intervention. Individuals who consumed less than 4 grams of dietary FODMAPs during the high FODMAP diet were excluded from this portion of the analysis. As a result, 8 participants were excluded from this portion of data analysis leaving a total of 8 participants for analysis.

Amongst 8 "compliant" participants, FODMAP intake was increased under the high FODMAP dietary intervention. Analysis showed a highly significant Treatment effect $(\mathrm{p}=0.001, \eta 2=0.829)$ and a non-significant treatment $*$ time trend $(\mathrm{F}(1,7 \mathrm{df})=$ 4.101, $\mathrm{p}=0.082, \eta 2=0.369$ ). Within treatment changes were not significant. See Figure 4 for further analysis.

Amongst the 8 "non-compliant" subjects, FODMAP intake was not effected by the dietary interventions. Non-significant treatment and treatment * time trends $\left(F(1,7 \mathrm{df})=0.082, \mathrm{p}=.782, \eta^{2}=.016\right.$ and $\left.\mathrm{F}(1,7 \mathrm{df}) 0.132, \mathrm{p}=.731, \eta^{2}=0.026\right) . \quad$ Likewise there were no within treatment differences during the low and high FODMAP interventions ( $2.14 \pm 2.79$ vs. $0.49 \pm 0.47$ and $1.61 \pm 2.13$ vs. $0.50 \pm 0.31$ respectively). Blood Glucose: 
Figure 5 shows blood glucose response amongst compliant participants. Blood glucose response was associated with significant treatment $(\mathrm{F}(1,7 \mathrm{df})=8.293, \mathrm{p}=0.024$, $\left.\eta^{2}=.542\right)$, Time $\left(F(1,7 \mathrm{df})=32.13, \mathrm{p}<0.000, \eta^{2}=0.821\right)$ and treatment $*$ time interaction ( $\left.F(1,7 \mathrm{df})=7.216, \mathrm{p}=0.007, \eta^{2}=0.508\right)$. Post-prandial blood glucose response under high FODMAP conditions was significantly lower 30 min after test meal when compared to the low FODMAP diet $(121.38 \pm 17.46$ vs. $148.25 \pm 27.54: \mathrm{t}=4.521, \mathrm{p}=.003)$. There were no within treatment differences amongst the two interventions. Paired t-tests showed a significantly lower TAUC response under high FODMAP conditions when compared to Low FODMAP conditions $(249.56 \pm 35.70$ vs. $219.25 \pm 2189, \mathrm{t}=3.603$, $\mathrm{p}=0.009)$.

\section{Subjective Appetite:}

Analysis of subjective appetite amongst the compliant sample showed no significant post-intervention differences between the high and low-FODMAP interventions. Regarding subjective hunger, treatment $(F(1,7 \mathrm{df})=0.856, \mathrm{p}=.386, \eta 2=$ $.109)$ and treatment $*$ time $(F(1,7 \mathrm{df})=.494, \mathrm{p}=.620, \eta 2=.066)$ were not significant with small effect sizes. Significant within treatment differences, regarding subjective hunger, were seen 30 minutes after administration of the test meal under the high FODMAP intervention $(14.13 \pm 15.75$ vs. $21.25 \pm 16.64, \mathrm{t}=-2.769, \mathrm{p}=0.028)$. Total AUC for subjective appetite did not show any differences between the high and lowFODMAP diets and are listed in table 4. Likewise, satiety was unaffected by the interventions as indicated by non-significant treatment $(F(1,7 \mathrm{df})=.140, \mathrm{p}=.719, \eta 2=$ $.020)$ and treatment $*$ time $(\mathrm{F}(1,7 \mathrm{df})=0.872, \mathrm{p}=.440, \eta 2=.111)$ interactions. Total AUC for subjective satiety was also unaffected. Prospective consumption remained 
unchanged throughout study as indicated by non-significant treatment $(\mathrm{F}(1,7 \mathrm{df})=.372$, $\mathrm{p}=.559)$ and treatment $*$ time $(\mathrm{F}(1,7 \mathrm{df})=.069, \mathrm{p}=.933)$ interactions.

\section{Discussion:}

\section{Summary:}

This study is novel because it was the first study to examine a possible prebiotic effect associated with a free-living, high-FODMAP diet. The purpose of this particular study was to examine how changes in dietary FODMAP consumption, amongst a healthy sample, in a free-living setting, would affect blood glucose and subjective appetite ratings in both fasting and post-prandial conditions. The results of this study indicate that there was limited dietary compliance during the highFODMAP intervention. This limited compliance resulted in non-significant differences regarding fasting breath hydrogen. There were no significant differences regarding blood glucose concentrations as a result of the dietary interventions. Partial eta squared was calculated and indicated that there was a small effect size between the two interventions $(0.155)$. However, the negative correlation seen between breath hydrogen and blood glucose is suggestive of a prebiotic effect.

To examine a possible prebiotic effect associated with dietary FODMAPs individuals with the highest total FODMAP intake were analyzed. Significant decreases regarding blood glucose response were seen in the sample of compliant individuals. No changes significant regarding subjective appetite were seen in the sample compliant with the high-FODMAP diet.

Effect of FODMAP intake on blood glucose response:

Fermentation of dietary carbohydrates by colonic microbiota has been found to influence blood glucose response through increased production of short-chain fatty 
acids (SCFA). SCFAs bind to L-cells lining the intestinal epithelium resulting in increased production of a variety of gut-peptide hormones, most notably glucagonlike-peptide $1^{38}$. Previous studies have shown that supplementation of fructooligosaccharides can increase concentrations of GLP-1 in the proximal colon by $50 \%{ }^{39}$. Binding of GLP-1 to beta cells increases expression of glucose transporters making the beta-cell more "sensitive" to the presence of extracellular glucose ${ }^{40,41}$. An increase in GLP-1 has been associated with increased activity of beta-cells in the presence of glucose ${ }^{40,42}$. Experimentally, increased endogenous production of GLP-1 has been inversely associated with blood glucose response amongst a healthy sample $^{20}$.

The present study analyzed blood glucose across the sample of 16 participants; however, no significant differences between the two dietary interventions were seen. This was likely due, in part, to poor dietary adherence under high FODMAP conditions. Amongst the sample of 16 subjects, the low FODMAP dietary intervention was successful in decreasing FODMAP intake from baseline assessment. Under the high FODMAP intervention, FODMAP intake increased by 4.52 grams over the low FODMAP diet. While this was a statistically significant difference, this discrepancy regarding FODMAP intake between the two diets was likely not large enough produce a prebiotic effect.

Dietary compliance amongst the sample was also considered. Individuals with the highest FODMAP intake throughout the high-FODMAP intervention $(n=8)$ were analyzed. Reanalysis of blood glucose for this sample showed a significantly reduced blood glucose response. Furthermore, this sample showed reduced post-prandial 
blood glucose response 30 minutes after administration of test meals. Previous studies show similar changes in post-prandial glycemic responses; however, these interventions were associated with considerably more FODMAP intake ${ }^{21,22}$. Brighenti et al. ${ }^{12}$ showed significantly reduced glycemic response amongst a sample consuming 5 grams of lactulose ${ }^{12}$. However it should be noted that these studies involved supplementation of FODMAPs. In order to achieve target FODMAP intake, individuals either consumed food in laboratory setting or were provided with food to consume outside of the laboratory settings. This particular dietary intervention only utilized dietary instruction and all choices regarding FODMAP intake were made by the participant in a free-living setting. These results suggest that a prebiotic effect in the form of significant changes in post-prandial glycemic response can be achieved with relatively small changes in FODMAP intake.

Importantly, indications of a prebiotic effect were not evident in the noncompliant sample. This sample consumed 0.50 grams of FODMAPs during the high FODMAP intervention, and no indication of a prebiotic effect was seen in the form of reduced glycemic response.

Determination of sample compliant with high-FODMAP diet:

Assessment of FODMAP intake for dietary compliance was performed in accordance to previous research ${ }^{4,33}$. The NDSR database utilized for quantification of FODMAP intake did not assess dietary oligosaccharides consumed. Assessment of total FODMAP intake included fructose, lactose, and sugar alcohols. Fructose and lactose are considered to be conditional FODMAPs due to the variation regarding their absorption amongst healthy populations. Fructose, for example, is absorbed through 
two transport mechanisms: the GLUT 2 and GLUT 5 transporters. The GLUT 5 transporter is specific for fructose, has a high affinity for the monosaccharide, however, its ability to absorb glucose is limited ${ }^{43}$. The GLUT 2 transporter absorbs fructose in conjunction with glucose; therefore dietary fructose is only completely absorbed when equimolar concentrations of glucose are present ${ }^{44,45}$. Thus in order to be considered a dietary FODMAP, fructose intake had to exceed that of glucose ${ }^{4,33}$. Lactose was not considered a FODMAP for the purposes of this study due to the selection criteria allowing only for individuals who had no diagnostic history of lactose intolerance and considered themselves able to consume lactose.

\section{Breath Hydrogen:}

In addition to SCFAs, hydrogen is another common byproduct of fermentation of malabsorbed carbohydrates. Hydrogen gas, produced in the colon, is absorbed through the walls of the intestine, entering the bloodstream, and is subsequently excreted as a component of exhaled breath ${ }^{46}$. Microbial fermentation of carbohydrates is considered the only means through which this "breath hydrogen" can be generated

29. Breath hydrogen testing is a reliable method of measuring the extent to which carbohydrates are being malabsorbed and fermented ${ }^{8,33,47}$. Changes in breath hydrogen concentration are indicative of quantity of FODMAPs consumed and also the rate of digestion. The ability of dietary FODMAPs to evoke a prebiotic effect has been intimately linked to changes in breath hydrogen ${ }^{46}$. This particular study found a non-significant increase in fasting breath hydrogen during the high- FODMAP intervention (2.11 vs. $6.36 \mathrm{ppm})$. While statistical significance was not obtained, the breath hydrogen values obtained under high-FODMAP conditions approached the 
upper limit of what would be expected for healthy individuals ${ }^{48}$. Importantly, this study did find an inverse relationship between fasting breath hydrogen values and blood glucose response indicating a close relationship between increased colonic fermentation and glycemic response. Furthermore, these findings are consistent to that of previous research ${ }^{20}$.

\section{Effect of intervention on subjective appetite:}

Gut peptide hormones have also been shown to impact subjective appetite. For example GLP-1 has also been shown to bind to afferent nerve fibers of the central nervous system resulting in increased feelings of satiety ${ }^{40,42}$. Intravenous infusion of GLP-1 in a healthy human sample resulted in increased feelings of "fullness" and satiety that were not seen in the control group $(\mathrm{p}<0.03)^{49}$. Peptide PYY is another gut peptide hormone found to impact subjective appetite. Obese individuals are subject to reduced endogenous PYY concentrations, and increases in endogenous PYY has been found to attenuate appetite ${ }^{50}$. Cani et al. ${ }^{22}$ showed increased colonic fermentation associated with FOS supplementation resulted in increased GLP-1 and PYY, and subsequent reductions in subjective hunger after a standardized test meal ${ }^{22}$. A recent study regarding supplementation of fructans, in a free-living sample, reduced subjective hunger ratings in a dose dependent manner $(\mathrm{p}<0.03)^{23}$. In the present study, there was no difference between the two dietary interventions regarding subjective appetite ratings perhaps due to insignificant dose.

\section{Strengths and limitations:}

The crossover design of this study was a major strength since each individual served as his or her own control and eliminated possible inequities in experimental and 
control groups that may exist in between group experimental designs. All outcomes in this study (blood glucose, cholesterol, and subjective appetite) were measured utilizing validated instrumentation.

Possible limitations of this study include the use of NDSR to analyze FODMAP intake. The inability of NDSR to quantify oligosaccharide consumption in our sample limited our ability to access dietary compliance. Future studies may consider alternative methods of assessing FODMAP intake ${ }^{51}$. Further limitations was our small sample of healthy participants, which limits the generalizability of our findings. Further work my consider utilizing subjects with, or at risk of type two diabetes.

\section{Conclusion:}

The dietary intervention, utilized throughout this study, was unsuccessful in increasing FODMAP intake sufficiently to produce a prebiotic effect in the sample of 16 healthy young adults. However, when examining data from individuals consuming greatest quantities of FODMAPs during the high FODMAP period of the intervention, a potential prebiotic effect was encountered as seen in reduced postprandial blood glucose response. This indicates a possible prebiotic potential of FODMAPs consumed in a diet of free-living individuals. However, longer-term studies, with larger compliant samples are needed to confirm these results. 


\section{References}

1. Biesiekierski JR, Rosella O, Rose R, et al. Quantification of fructans, galactooligosacharides and other short-chain carbohydrates in processed grains and cereals. J Hum Nutr Diet. 2011;24(2):154-176.

2. Gibson PR, Shepherd SJ. Evidence-based dietary management of functional gastrointestinal symptoms: The FODMAP approach. J Gastroenterol Helatol. Feb 2010;25(2):252-258.

3. Barrett JS. Extending Our Knowledge of Fermentable, Short-Chain Carbohydrates for Managing Gastrointestinal Symptoms. Nutr Clin Pract. June 1, 2013 2013;28(3):300-306.

4. Barrett JS, Gearry RB, Muir JG, et al. Dietary poorly absorbed, short-chain carbohydrates increase delivery of water and fermentable substrates to the proximal colon. Aliment Pharmacol Ther. Apr 2010;31(8):874-882.

5. Barrett JS, Shepherd SJ, Gibson PR. Strategies to manage gastrointestinal symptoms complicating enteral feeding. JPEN. J Parenter Enteral Nutr. JanFeb 2009;33(1):21-26.

6. Marciani L, Cox EF, Hoad CL, et al. Postprandial changes in small bowel water content in healthy subjects and patients with irritable bowel syndrome. Gastroenterology. Feb 2010;138(2):469-477, 477 e461.

7. Born P. The clinical impact of carbohydrate malabsorption. Arab J Gastroenterol. Mar 2011;12(1):1-4.

8. Fernandez-Banares F, Rosinach M, Esteve M, Forne M, Espinos J, Viver JM. Sugar malabsorption in functional abdominal bloating. Gastroenterology. Apr 2005;128(4):A331-A332.

9. Gibson PR, Barrett JS, Muir JG. Functional bowel symptoms and diet. Intern Med J. Oct 2013;43(10):1067-1074.

10. Brand-Miller JC. Glycemic Load and Chronic Disease. Nutrition Reviews. 2003;61:S49-S55.

11. Barclay AW, Petocz P, McMillan-Price J, et al. Glycemic index, glycemic load, and chronic disease risk - a meta-analysis of observational studies. Am J Clin Nutr. March 1, 2008 2008;87(3):627-637. 
12. Brighenti F, Benini L, Del Rio D, et al. Colonic fermentation of indigestible carbohydrates contributes to the second-meal effect. Am J Clin Nutr. Apr 2006;83(4):817-822.

13. Wolever TM, Jenkins DJ, Ocana AM, Rao VA, Collier GR. Second-meal effect: low-glycemic-index foods eaten at dinner improve subsequent breakfast glycemic response. Am J Clin Nutr. October 1, 1988 1988;48(4):1041-1047.

14. Liljeberg H, Bjorck I. Effects of a low-glycaemic index spaghetti meal on glucose tolerance and lipaemia at a subsequent meal in healthy subjects. Eur J Clin Nutr. Jan 2000;54(1):24-28.

15. Liljeberg HG, Akerberg AK, Bjorck IM. Effect of the glycemic index and content of indigestible carbohydrates of cereal-based breakfast meals on glucose tolerance at lunch in healthy subjects. Am J Clin Nutr. Apr 1999;69(4):647-655.

16. Nilsson A, Granfeldt Y, Ostman E, Preston T, Bjorck I. Effects of GI and content of indigestible carbohydrates of cereal-based evening meals on glucose tolerance at a subsequent standardised breakfast. Eur J Clin Nutr. Sep 2006;60(9):1092-1099.

17. Cani PD, Knauf C, Iglesias MA, Drucker DJ, Delzenne NM, Burcelin R. Improvement of Glucose Tolerance and Hepatic Insulin Sensitivity by Oligofructose Requires a Functional Glucagon-Like Peptide 1 Receptor. Diabetes. May 1, 2006 2006;55(5):1484-1490.

18. Priebe MG, Wang H, Weening D, Schepers M, Preston T, Vonk RJ. Factors related to colonic fermentation of nondigestible carbohydrates of a previous evening meal increase tissue glucose uptake and moderate glucose-associated inflammation. Am J Clin Nutr. Jan 2010;91(1):90-97.

19. Nilsson AC, Ostman EM, Granfeldt Y, Bjorck IM. Effect of cereal test breakfasts differing in glycemic index and content of indigestible carbohydrates on daylong glucose tolerance in healthy subjects. Am J Clin Nutr. Mar 2008;87(3):645-654.

20. Nilsson AC, Östman EM, Holst JJ, Björck IME. Including Indigestible Carbohydrates in the Evening Meal of Healthy Subjects Improves Glucose Tolerance, Lowers Inflammatory Markers, and Increases Satiety after a Subsequent Standardized Breakfast. J Nutr. April 1, 2008 2008;138(4):732739.

21. Nilsson A, Johansson E, Ekstrom L, Bjorck I. Effects of a Brown Beans Evening Meal on Metabolic Risk Markers and Appetite Regulating Hormones 
at a Subsequent Standardized Breakfast: A Randomized Cross-Over Study. Plos One. Apr 5 2013;8(4).

22. Cani PD, Lecourt E, Dewulf EM, et al. Gut microbiota fermentation of prebiotics increases satietogenic and incretin gut peptide production with consequences for appetite sensation and glucose response after a meal. Am J Clin Nutr. Nov 2009;90(5):1236-1243.

23. Pedersen C, Lefevre S, Peters V, et al. Gut hormone release and appetite regulation in healthy non-obese participants following oligofructose intake. A dose-escalation study. Appetite. Jul 2013;66:44-53.

24. Rosen LA, Ostman EM, Bjorck IM. Effects of cereal breakfasts on postprandial glucose, appetite regulation and voluntary energy intake at a subsequent standardized lunch; focusing on rye products. Nutr J. 2011;10:7.

25. Flint A, Raben A, Blundell JE, Astrup A. Reproducibility, power and validity of visual analogue scales in assessment of appetite sensations in single test meal studies.Int J Obes Relat Metab Disord. Jan 2000;24(1):38-48.

26. Jonnalagadda SS, Mitchell DC, Smiciklas-Wright H, et al. Accuracy of energy intake data estimated by a multiple-pass, 24-hour dietary recall technique. $J$ Am Diet Assoc. Mar 2000;100(3):303-308; quiz 309-311.

27. Riby JE, Fujisawa T, Kretchmer N. Fructose absorption. Am J Clin Nutr. November 1, 1993 1993;58(5):748S-753S.

28. Grant JD, Bezerra JA, Thompson SH, Lemen RJ, Koldovsky O, Udall JN, Jr. Assessment of lactose absorption by measurement of urinary galactose. Gastroenterology. Oct 1989;97(4):895-899.

29. Simren M, Stotzer PO. Use and abuse of hydrogen breath tests. Gut. Mar 2006;55(3):297-303.

30. Methodology and Indications of H2-Breath Testing in Gastrointestinal Diseases: the Rome Consensus Conference. Aliment Pharmacol Ther. 2009;29:1-49.

31. Lee W, Davidson G, Moore D, Butler R. Analysis of the breath hydrogen test for carbohydrate malabsorption: Validation of a pocket-sized breath test analyser. J paediatr Child Health. 2000;36(4):340-342.

32. Lohman TG, Roche AF, Martorell R. Anthropometric standardization reference manual. Champaign, IL: Human Kinetics Books; 1988. 
33. Ong DK, Mitchell SB, Barrett JS, et al. Manipulation of dietary short chain carbohydrates alters the pattern of gas production and genesis of symptoms in irritable bowel syndrome. J Gastroenterol Helatol. Aug 2010;25(8):13661373.

34. Shepherd SJ, Gibson PR. Fructose malabsorption and symptoms of irritable bowel syndrome: guidelines for effective dietary management. Journal of the American Dietetic Association. Oct 2006;106(10):1631-1639.

35. Staudacher HM, Whelan K, Irving PM, Lomer MC. Comparison of symptom response following advice for a diet low in fermentable carbohydrates (FODMAPs) versus standard dietary advice in patients with irritable bowel syndrome. J Hum Nutr Diet : the official journal of the British Dietetic Association. Oct 2011;24(5):487-495.

36. Grubbs F. Procedures for Detecting Outlying Observations in Samples. Technometrics. // 1969;11(1).

37. Cohen J. Statistical Power Analysis. Cur Dir Psychol Sci. 1992;1(3):98-101.

38. Darzi J, Frost GS, Robertson MD. Do SCFA have a role in appetite regulation? Proc Nutr Soc. 2011;70(01):119-128.

39. Cani PD, Neyrinck AM, Maton N, Delzenne NM. Oligofructose Promotes Satiety in Rats Fed a High $\square$ Fat Diet: Involvement of Glucagon $\square$ Like Peptide $\square$ 1. Obesity research. 2005;13(6):1000-1007.

40. Baggio LL, Drucker DJ. Biology of incretins: GLP-1 and GIP. Gastroenterology. May 2007;132(6):2131-2157.

41. Stipanuk MH, Caudill MA. Biochemical, Physiological, and Molecular Aspects of Human Nutrition. Elsevier Health Sciences; 2013.

42. Neary MT, Batterham RL. Gut hormones: Implications for the treatment of obesity. Pharmacology \& Therapeutics. 10// 2009;124(1):44-56.

43. Jones HF, Butler RN, Brooks DA. Intestinal fructose transport and malabsorption in humans. Am J Physiol Gastrointest Liver Physiol. Feb 2011;300(2):G202-206.

44. Nanda R, Shu LH, Thomas JR. A FODMAP Diet Update: Craze or Credible? Prac Gastroenterol. 2012:37.

45. Ravich WJ, Bayless TM, Thomas M. Fructose: incomplete intestinal absorption in humans. Gastroenterology. Jan 1983;84(1):26-29. 
46. Eisenmann A, Amann A, Said M, Datta B, Ledochowski M. Implementation and interpretation of hydrogen breath tests. J Breath Res. Dec 2008;2(4):046002.

47. Barrett JS, Gibson PR. Fermentable oligosaccharides, disaccharides, monosaccharides and polyols (FODMAPs) and nonallergic food intolerance: FODMAPs or food chemicals? Therap Adv Gastroenterol. 2012;5(4):261-268.

48. Perman JA, Modler S, Barr RG, Rosenthal P. Fasting breath hydrogen concentration: normal values and clinical application. Gastroenterology. Dec 1984;87(6):1358-1363.

49. Flint A, Raben A, Astrup A, Holst JJ. Glucagon-like peptide 1 promotes satiety and suppresses energy intake in humans. J Clin Invest. Feb 1 1998;101(3):515520.

50. Karra E, Batterham RL. The role of gut hormones in the regulation of body weight and energy homeostasis. Mol Cell Endocrinol. 3/25/ 2010;316(2):120128.

51. Barrett JS, Gibson PR. Development and Validation of a Comprehensive Semi-Quantitative Food Frequency Questionnaire that Includes FODMAP Intake and Glycemic Index. J Am Diet Assoc. 10// 2010;110(10):1469-1476. 


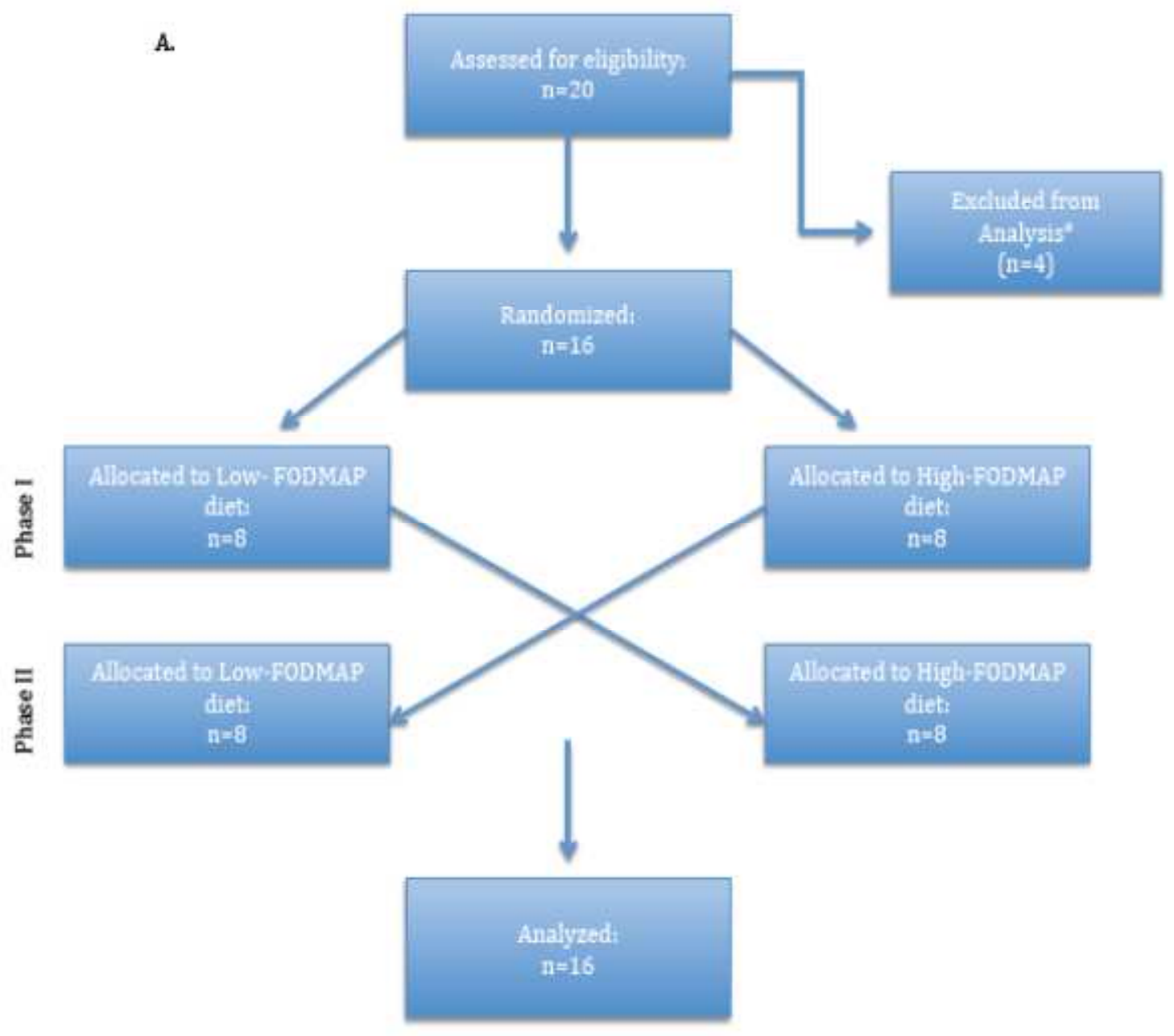

B) Laboratory Data Collection:

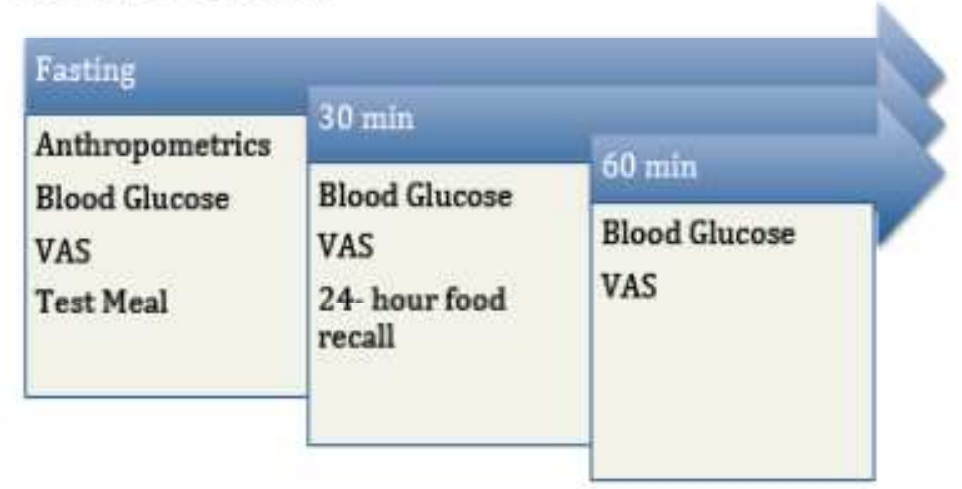

Figure 1: A. Progression of individuals through phases of this study. Phase I and II were separated by 11-day washout period. * Indicates that four subjects withdrew from this study, one for medical conditions unrelated to the study and three failed to compete any assessments beyond baseline. B) Laboratory Data collection. Occurring Tuesday (Baseline) and Friday (Post- Intervention). VAS, Visual analogue scale. 
Table 1: Demographics, $\mathrm{n}=16$

\begin{tabular}{lr} 
Variable & \\
Female, $\mathbf{n}(\%)$ & $10(62.5)$ \\
Male, $\mathbf{n}(\%)$ & $6(37.5 \%)$ \\
Age $(\mathbf{y r}), \mathbf{M} \pm$ SD & $20.47 \pm 1.77$ \\
Body Weight $(\mathbf{k g}), \mathbf{M} \pm \mathbf{S D}$ & $63.85 \pm 11.65$ \\
Body Mass Index $\left(\mathbf{k g} / \mathbf{m}^{2}\right), \mathbf{M} \pm \mathbf{S D}$ & $22.21 \pm 2.45$ \\
Waist Circumference $(\mathbf{c m}), \mathbf{M} \pm \mathbf{S D}$ & $78.98 \pm 7.57$ \\
Body Fat Percent $(\boldsymbol{\%}) \boldsymbol{M}, \mathbf{M D}$ & $18.80 \pm 10.37$ \\
\hline
\end{tabular}

*body fat percent obtained via BOD POD Body Composition System (Life Measurement Instruments, Concord, Calif., USA) 


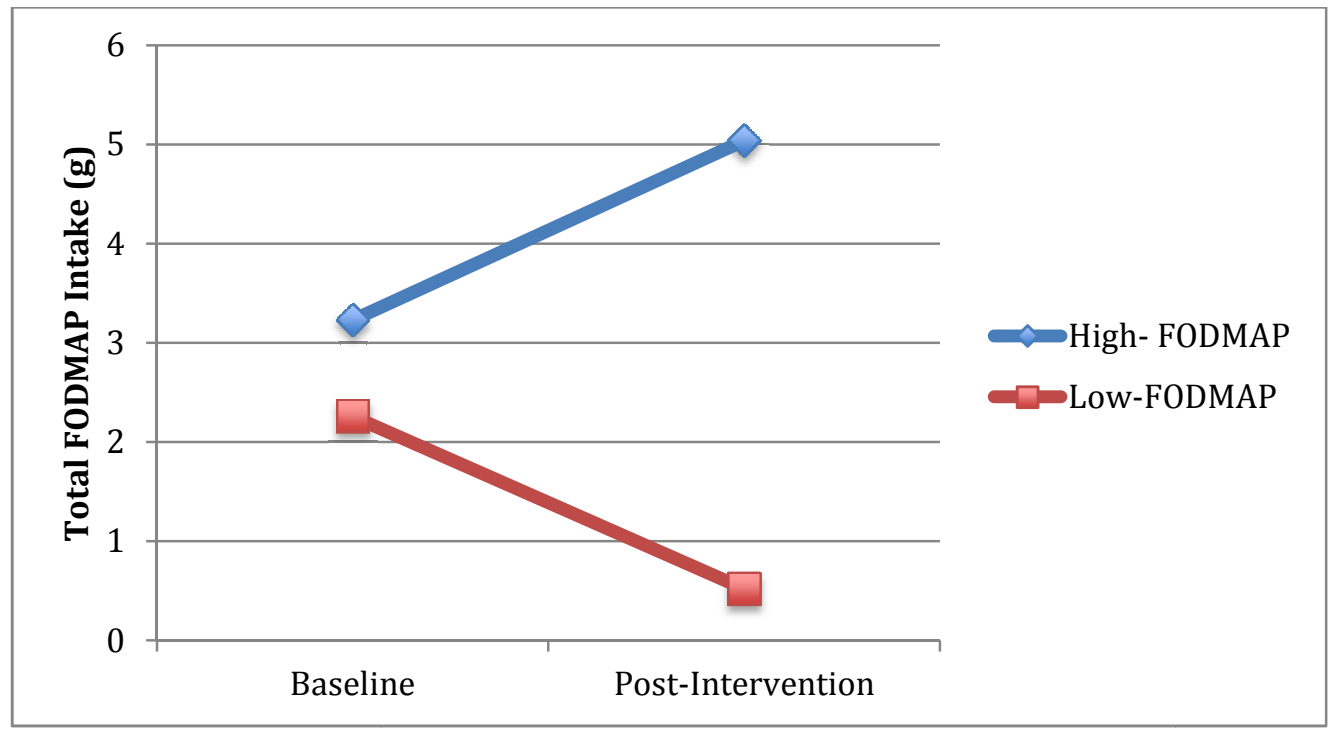

Figure 2: FODMAP consumption $(\mathrm{n}=16)$ excluding the conditional FODMAP lactose. Fructose was only included when consumed in excess of glucose. Significant Treatment $(F(1,14 \mathrm{df})=9.845, \mathrm{p}<0.01, \eta 2=0.395))$ and treatment $*$ time effects were noted $\left(F(1,14 \mathrm{df})=5.315, \mathrm{p}<0.05, \eta^{2}=0.262\right)$. 
A).

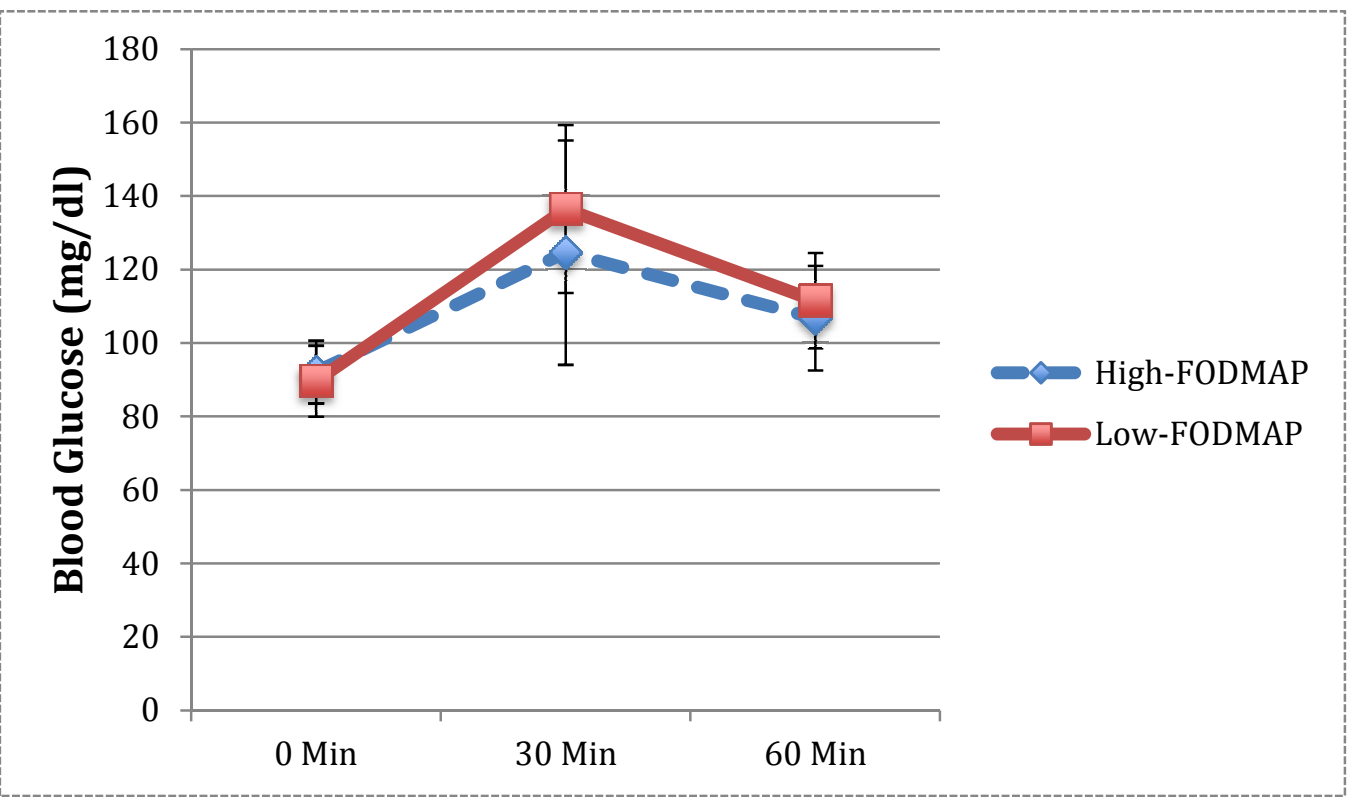

B).

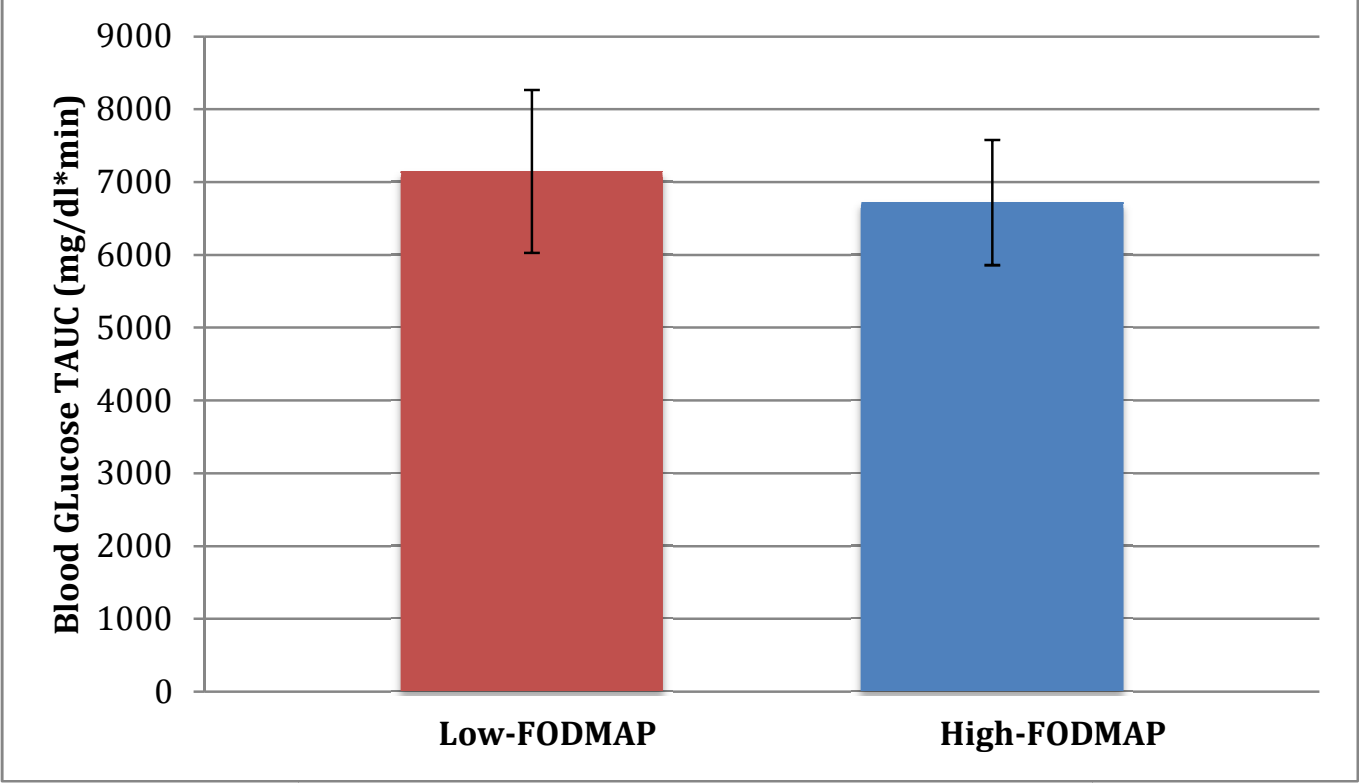

Figure 3: A) Post-intervention blood glucose concentrations (Mean \pm SD) and B) corresponding total area under the curve. Data is from $n=14$ subjects. A) Treatment and treatment * time interactions were not significant as determined by a repeated measures ANOVA $\left(p=0.224 \eta^{2}=0.111\right.$ and $p=0.111 \eta^{2}=0.155$ respectively). There was a significant effect of time $(\mathrm{p}<.001)$. B) Shows total area under the curve for both treatments. B). Non-significant treatment, time, and treatment by time interactions noted between the two treatments $(\mathrm{p}=0.318,0.473,0.324$ respectively). 
Table 2. Subjective appetite expressed as TAUC $^{1}($ mean \pm sd $)$

\begin{tabular}{|c|c|c|c|c|c|c|c|c|}
\hline & \multicolumn{3}{|c|}{$\underline{\text { Low-FODMAP diet }}$} & \multicolumn{3}{|c|}{ High-FODMAP diet } & \multicolumn{2}{|c|}{$\underline{\text { ANOVA }}^{2}$} \\
\hline & Baseline & Post & $\Delta$ & $\underline{\text { Baseline }}$ & Post & $\Delta$ & p-value & $\eta^{2}$ \\
\hline Hunger & $1635 \pm 1041$ & $1959 \pm 806$ & 324 & $1739 \pm 1059$ & $2079 \pm 894$ & 340 & .655 & .025 \\
\hline Satiety & $3516 \pm 865$ & $3317 \pm 839$ & -199 & $3615 \pm 857$ & $3279 \pm 1006$ & -336 & .819 & .013. \\
\hline Prospective Consumption & $1830 \pm 1152$ & $1796 \pm 590$ & -34 & $1687 \pm 800$ & $2006 \pm 765$ & 328 & .958 & .003 \\
\hline
\end{tabular}

1:TAUC for visual analogue scales is expressed as $(\mathrm{mm} * \mathrm{~min})$ for the sample of $n=16$

$\omega \quad 2$ : indicates use of $2 \times 2$ repeated measures ANOVA. P-value and $\eta^{2}$ are for treatment*time interaction 
Figure 4: FODMAP intake (excluding conditional FODMAPs) $n=8$

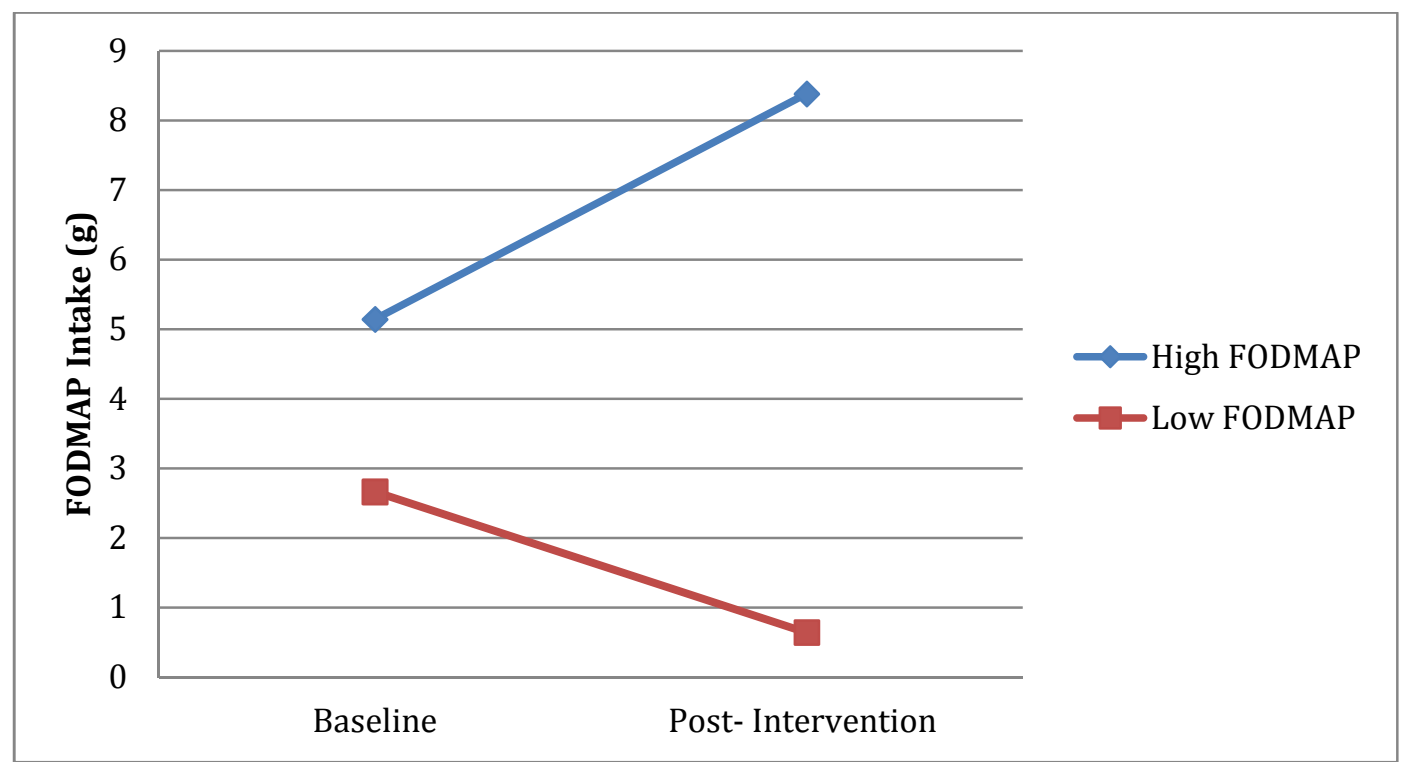

FODMAP consumption excluding conditional FODMAP lactose. Fructose was only included when consumed in excess of glucose. Only includes individuals who were compliant (consumed $>4$ grams of FODMAPs as estimated from 24-hr Recall). Significant treatment effect (Treatment: $F(1,7 \mathrm{df})=$ $\left.33.875, \mathrm{p}=0.001, \square^{2}=0.829\right)$. A non-significant treatment $*$ time trend was noted $(\mathrm{F}(1,7 \mathrm{df})=$ $4.101, \mathrm{p}=0.082, \square=0.369$ ) 
A).

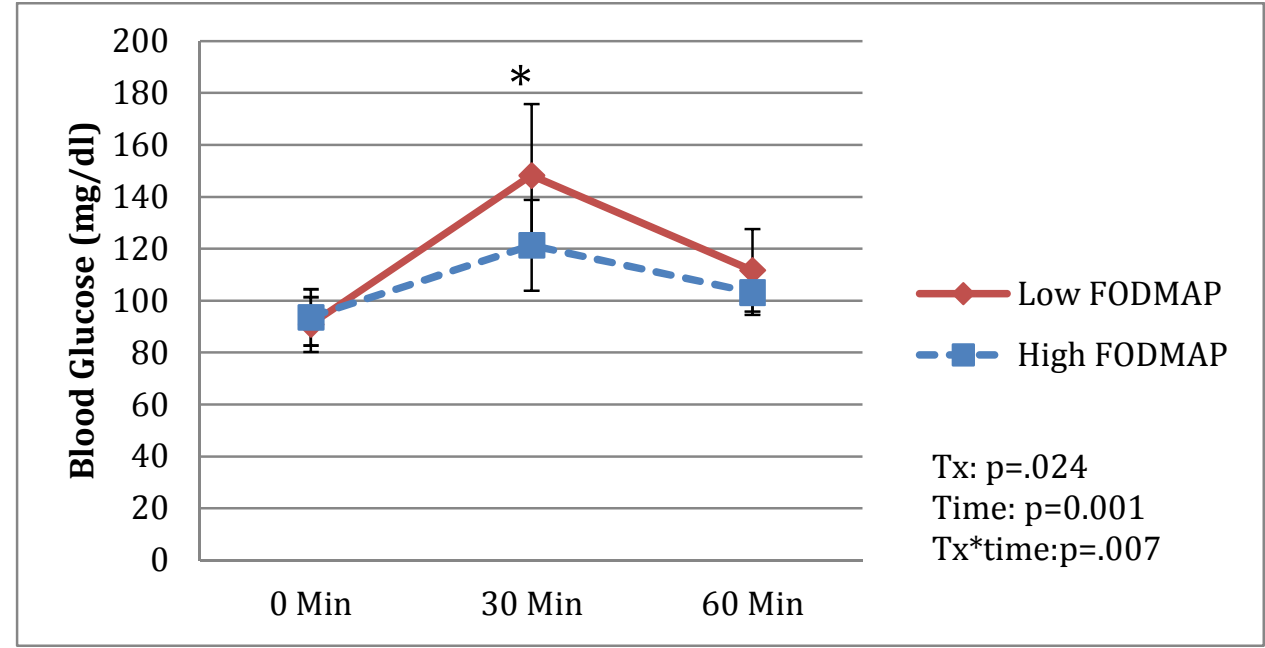

B).

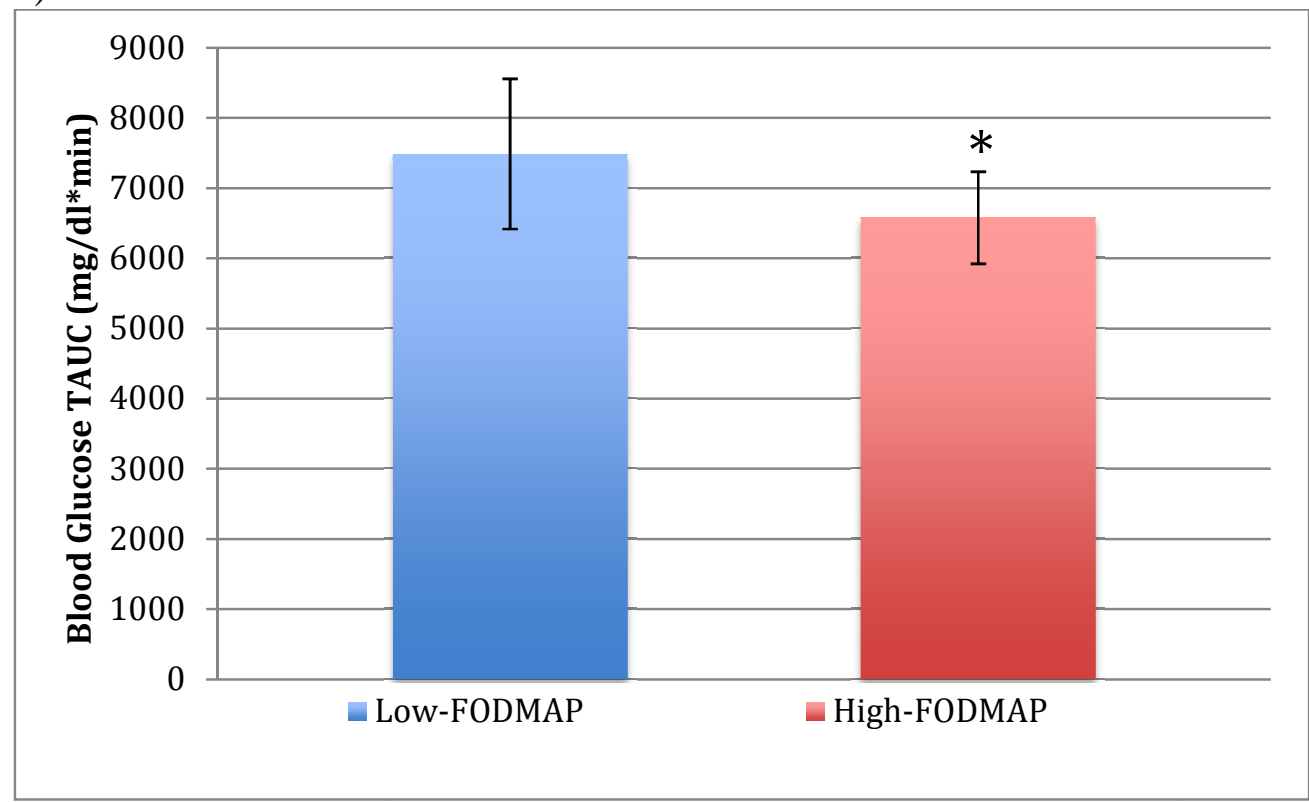

Figure 5: A) Blood glucose concentrations (post-intervention) comparing high and low FODMAP conditions (Mean $\pm \mathrm{SD}$ ). Data is from $\mathrm{n}=8$ participants who were considered to be compliant with highFODMAP diet. Treatment, Time, and Treatment* time * interactions were all significant as determined by repeated measures ANOVA. Post-prandial blood glucose response 30 min after test meal significantly lower under high- FODMAP conditions $(\mathrm{p}=.003)$. B) Total AUC was significantly lower under high-FODMAP conditions ( $\mathrm{p}=.009$ ).

* Indicates statistically significant decrease under high FODMAP conditions when compared to low $(\mathrm{P}<0.01)$. 
Table 3. subjective appetite expressed as TAUC ${ }^{1}$

\begin{tabular}{|c|c|c|c|c|c|c|c|c|}
\hline & \multicolumn{3}{|c|}{ Low-FODMAP diet } & \multicolumn{3}{|c|}{ High-FODMAP diet } & \multicolumn{2}{|c|}{ ANOVA $^{2}$} \\
\hline Hunger & $1830 \pm 1152$ & $1796 \pm 590$ & -34 & $1687 \pm 800$ & $2006 \pm 765$ & 319 & $\begin{array}{l}.344 \\
.135\end{array}$ & $\begin{array}{c}.128 \\
.289\end{array}$ \\
\hline Satiety & $3476 \pm 1124$ & $3810 \pm 414$ & -334 & $3960 \pm 893$ & $3650 \pm 700$ & -310 & & \\
\hline Prospective Consumption & $2023 \pm 1202$ & $1996 \pm 1042$ & -27 & $1850 \pm 1213$ & $2150 \pm 1077$ & 300 & .402 & .080 \\
\hline
\end{tabular}

1: TAUC for visual analogue scale is expressed as ( $\left.\mathrm{mm}^{*} \mathrm{~min}\right)$ for the sample of $n=8$

2: indicates the use of $2 \times 2$ repeated measures ANOVA. P-value and $\eta^{2}$ are for treatment*time interactions. 


\section{APPENDICIES}

\section{A. REVIEW OF THE LITERATURE}

\section{Overview:}

Approximately 100 trillion bacterial cells colonize the Human colon. ${ }^{57}$. Recent research indicates that the metabolic activity of this colonic microbiota can make a significant contribution to the overall health and well being of the host organism ${ }^{57,58}$. Dietary components, mostly carbohydrates and some proteins, that evade digestion and absorption in the small intestine are subsequently deposited in the colon and are subject to the metabolic activity of the resident colonic microbiota. Research indicates that bacterial metabolism of dietary carbohydrates can have important implications regarding a wide variety of metabolic processes in the human body. Fermentable oligosaccharides, disaccharides, monosaccharaides and polyols (FODMAPs) are a class of carbohydrate that evade enzymatic hydrolysis in the small intestine and are subsequently metabolized via fermentation by colonic microbiota ${ }^{7,59}$. The chemical properties of FODMAPS along with byproducts of their fermentation are known to exacerbate symptomology associated with certain functional gastrointestinal disorders, most notably irritable bowel syndrome (IBS) ${ }^{7,59,60}$. However, colonic fermentation of FODMAPs has also been linked to a number of health benefits most notably improved blood glucose response, decreased appetite, and prolonged periods of satiety. Thus dietary FODMAPs could play an important clinical role regarding a wide variety of metabolic disorders. 


\section{Characteristics and Dietary Sources of FODMAPs:}

Carbohydrates are a diverse class of macromolecule. Polymeric forms of carbohydrates are classified by molecular size, degree of polymerization, individual monomers making up polysaccharides, as well as the types of linkages between monomers ${ }^{61}$. FODMAPs are often characterized by consisting of beta-glycosidic linkages with a low degree of polymerization (>10 subunits) distinguishing them from longer chained polysaccharides and dietary fibers ${ }^{60}$. These unique short chain carbohydrates are common in a wide variety of foods that are often consumed in a modern western diet ${ }^{60,62}$. Polymers of fructose, galactose, as well as sugar alcohols are amongst the most commonly consumed FODMAPs in a modernized diet ${ }^{62}$.

Fructans or fructo-oligosaccharides (FOS) are amongst the most common dietary FODMAPs ${ }^{47,62}$. Dietary sources of FOS are wheat products, some fruits (apples and pears), as well as honey ${ }^{63}$. Fructans are also commonly seen in grains and pasta products most notably wheat pasta, gluten-free pasta, and quinoa pasta ${ }^{1}$. The ability of the small intestine to absorb dietary FOS is severely limited ${ }^{64}$. Experimental evidence indicates that up to $89 \%$ of ingested fructans reach the colon intact $^{64}$. Thus the energy production associated with fructans is estimated to be 9.5 $\mathrm{kJ} / \mathrm{g}$ which is about half of that of sucrose ${ }^{64}$.

Galactans or Galacto-oligosaccharides are another common FODMAP present in modern diets. Synthesized via enzymatic reaction from lactose, galactooligosaccharides are composed of a chain of galactose monomers with a terminal glucose $^{65}$. GOS are associated with a degree of polymerization of between 2-8 monomeric subunits ${ }^{65}$. Beta-glycosidic bonds connecting these galactose monomers 
result in $90 \%$ of ingested GOS passing through the small intestine to the colon ${ }^{66}$.

Dietary sources of GOS include soy products, dried beans and peas, lentils humus, and are also associated with coffee consumption ${ }^{67}$. The amount of GOS contained in the diet is highly variable and is often dependent on the intake of legumes ${ }^{61,62}$. Galactan consumption is associated with a high prevalence of malabsorption in the small intestine and are thus categorized as FODMAPs.

Consumption of sugar alcohols (polyols) has been growing in prevalence in modernized society. The human small intestine lacks enzymes capable of hydrolyzing or absorbing polyols and experimental evidence indicates that as much as 70 percent of ingested polyols are malabsorbed ${ }^{68}$. Due to this high rate of malabosrption, polyols are considered to be a "low-calorie sweetener" providing approximately $2.4 \mathrm{kCals} / \mathrm{g}$ " Furthermore, polyols, such as sorbitol and mannitol have been associated with a similar "taste-profile" to other carbohydrates such as sucrose and are utilized commercially as a low calorie alternative to sucrose. Sorbitol and mannitol are featured in a variety of products such as chewing gum, mints, tooth pastes, and sweetened beverages ${ }^{63,69}$. Polyols also exist in nature in plants and function as osmoregulatory agents as well as an energy source ${ }^{68}$. Polyols are naturally derived from fruits such as apples, pears, and apricots and are associated with a variety of vegetables such as cauliflower, sweet corn, snow peas and mushrooms ${ }^{61,63,67}$.

\section{Malabsorption of FODMAPs:}

Malabsorption of macronutrients is regarded as the inability of the digestive system to enzymatically hydrolyze and absorb nutrients in the small intestine. On one hand, carbohydrates associated with $\alpha$-glycosidic linkages are readily absorbed by the 
human body ${ }^{70,71}$. Salivary and luminal hydrolase enzymes readily cleave these bonds allowing for absorption of these carbohydrates through transport proteins located in the first mucosal membrane of the small intestine ${ }^{70}$. These hydrolase enzymes are unable, however, to hydrolyze the $\beta$-glycosidic linkages that are associated with FODMAPs, thus inhibiting digestion and absorption of these carbohydrates ${ }^{70}$.

The lumen of the small intestine presents a substantial barrier regarding the rapid diffusion of large water-soluble molecules into enterocytes ${ }^{72}$. While some absorption of dietary nutrients occurs through passive diffusion, the absorption of dietary carbohydrates is largely dependent on carbohydrate transporters located in the enterocytes of the small intestine ${ }^{72}$. Both active transport and co-transport mechanisms are employed as a means of absorbing dietary carbohydrates. These transport proteins are highly specific and are often limited in the amount of certain carbohydrates that can be absorbed. For example, dietary fructose that is consumed in its monomeric form can also act as a "conditional" FODMAP. Fructose can be absorbed through two mechanisms: GLUT-5 transporter and the GLUT-2 transporter ${ }^{27}$. The GLUT-5 transporter is specific for fructose, however, its ability to absorb dietary fructose is limited ${ }^{27,48}$. While in the GLUT-2 transporter, fructose is absorbed in conjunction with glucose; therefore dietary fructose is only completely absorbed when equimolar concentrations of glucose are present ${ }^{47}$. Thus diets that are associated with a fructose intake that exceeds that of glucose are associated with malabsorption. Even in healthy individuals, dietary consumption of fructose that exceeds 25 grams is often associated with malabsorption indicating that dietary fructose is only partially absorbed in the small intestine ${ }^{7}$. 


\section{Osmotic Effect:}

One of the most notable characteristics of dietary FODMAPs is their ability to exert an osmotic effect on the gastrointestinal system. High quantities of dietary fructo-oligosaccharides, galacto-oligosaccharides, and polyols, act to draw water in to the lumen of the small intestine through simple diffusion ${ }^{6,63}$. Supplementation of a $5 \%$ solution of mannitol in healthy individuals was shown to increase luminal small intestinal water concentration $50 \mathrm{~mL}$, while supplementation of glucose was found to stimulate absorption of $147 \mathrm{~mL}$ of water in the small intestine $(\mathrm{P}<0.0001)^{6}$. The ability of dietary FODMAPs to exert this osmotic effect can have important implications regarding luminal distension, abdominal pain, and diarrhea that are associated with high levels of dietary FODMAPs ${ }^{2}$.

Barrett et al. ${ }^{5}$ examined the osmotic effect of a high FODMAP meal in in subjects with ileostomy. Throughout this analysis individuals consumed a high FODMAP test meal, and ileostomy effluent was collected and analyzed for FODMAP content. Utilizing this "ileostomy model," it was confirmed that an average of $32 \%$ (95\% CI, 6-73\%) of FODMAPs consumed escape digestion in the small intestine ${ }^{5}$. Furthermore, increases in FODMAPs found in ileostomy effluent were associated with a $22 \%(95 \% \mathrm{CI}, 5 \%-39 \%)$ increase in water content, indicating that malabsorbed

carbohydrates are osmotically active ${ }^{5,50}$. This analysis confirms the ability of a high FODMAP diet to exert an osmotic effect on the small intestine and establishes a mechanism through which these carbohydrates can affect gastrointestinal tolerance. Influence of FODMAPs on Orocecal Transit: 
The ability of dietary FODMAPs to exert an increased osmotic effect can have important implications regarding orocecal transit. The ability of FODMAPs to draw water into the lumen of the intestine results in increased peristaltic activity increasing the rate at which food travels through the gastrointestinal tract ${ }^{73}$. Ingestion of $25 \mathrm{~g}$ of fructose and $5 \mathrm{~g}$ of sorbitol was found to have a significantly higher rate of intestinal transit when compared to control $(\mathrm{P}=0.0033)^{73}$. Furthermore, the length of carbohydrate polymers was found to significantly effect orocecal-transit. Short chain carbohydrates (degree of polymerization $>10$ ), that are malabsorbed, have been found to have reduced orocecal transit times when compared to longer chained carbohydrates (degree of polymerization $<10)^{74}$. This shows that FODMAP consumption can alter the function of the small intestine and deliver ingested nutrients to the colon at a higher rate than longer chained, less osmiotically active carbohydrates.

\section{Rapid Fermentation of Malabsorbed FODMAPs:}

Malabsorbed carbohydrates are subsequently deposited in the proximal colon where they serve as substrate for microbial metabolism. Colonic microbiota utilize fermentation reactions to metabolize FODMAPs, which is characterized by increases in hydrogen gas formed in the colon. In conditions associated with low dietary FODMAP consumption, production on hydrogen gas by colonic micro flora is low. However, increases in dietary FODMAPs will result in measurable increases in hydrogen production ${ }^{75,76}$. Hydrogen gas that is formed through this process is absorbed through the wall of the colon where it enters the blood stream. Once it enters the blood stream, the hydrogen gas is subsequently excreted through the lungs ${ }^{75,76}$. 
Thus any changes in breath hydrogen concentrations can be attributed to colonic fermentation ${ }^{75,76}$.

Due to their short-chain length and ability to exert an osmotic effect, dietary FODMAPS are readily fermented by colonic microbiota. The ability of a carbohydrate to exert an osmotic force is inversely proportional to its degree of polymerization ${ }^{77}$. Thus the ability of FODMAPs to draw fluids in to the lumen of the small intestine enhanced over longer chained polysaccharides ${ }^{77}$. This increase in osmotic force "increases surface area available for enzymatic attack" making FODMAPs more susceptible to bacterial metabolism ${ }^{78}$. A recent single blind crossover analysis showed that FODMAP consumption significantly influenced breath hydrogen levels (low FODMAP diet, $43 \pm 18$ vs. high FODMAP diets $181 \pm 79$ ppm; $\mathrm{P}<0.0001)^{34}$. Changes in breath hydrogen concentration are indicative of quantity of FODMAPs consumed and also the rate of digestion ${ }^{75,79}$. Thus measurement of breath hydrogen can have important applications when assessing an individual's postprandial physiological response to FODMAP consumption.

\section{Role of FODMAPs in IBS:}

The poor digestion, osmotic effect and high rate of bacterial metabolism that characterize dietary FODMAPs have important implications regarding symptomology associated with irritable bowel syndrome. FODMAP metabolism has been found to induce luminal distension, abdominal pain, bloating, and distension ${ }^{2}$. Diets that minimize FODMAP consumption have been associated with reduction in symptoms associated with IBS ${ }^{37}$. Staudacher et al. ${ }^{37}$ found that $76 \%$ of IBS patients $(n=43)$ placed on a low FODMAP diet reported improvements in symptoms when compared 
to a healthy control group $(49 \% ; \mathrm{P}<0.001)^{37}$. Another analysis showed that longterm implementation of a low FODMAP diet resulted in significant clinical improvements in $81 \%$ of IBS patients over a one month period ${ }^{9}$. This clinical improvement was maintained in $66.7 \%$ of patients ${ }^{9}$. Symptomatic relief is directly correlated to decreased levels of breath hydrogen suggesting that carbohydrate malabsorption could be clinically significant in the provocation of gastrointestinal symptoms ${ }^{9,34}$.

\section{Potential prebiotic effect of FODMAPs:}

There is a multitude of different species and strains of bacteria that colonize the human colon. Some species of microbiota such as bifidobacteria and lactobacilli are beneficial to the overall health of the host organism yet others (eg. Clostridium) can negatively affect the overall health ${ }^{80}$. Consumption of poorly digested polysaccharides and oligosaccharides have shown to promote the development of these types of "healthy" colonic microbiota. Gibson and Roberfroid ${ }^{81}$ defined prebiotics as 'non-digestible food ingredients that selectively stimulate growth and/or activity of one or a limited number of bacteria in the colon, thereby improving host health ${ }^{81}$. These alterations in the colonic microbiota can have important implications regarding a number of risk factors regarding development of metabolic disease ${ }^{80}$.

The ability of FOS to exert a prebiotic effect has been studied rather extensively. FOS supplemented in bacterial culture of 21 different strains of bifidobacterium was associated with significant bacterial growth. Almost all of the strains of bifidobacterium were able to grow on a medium containing FOS, while only 8 strains were able to show significant growth on mediums containing the longer chain 
inulin ${ }^{82}$. Fructooligosaccharides have also proven to be an effective prebiotic in human models as well. Fecal bacterial concentrations, in humans, were found to have a dose-dependent relationship regarding oligofructose supplementation $(p<.009){ }^{83}$. This indicates that supplementation of FOS can be advantageous regarding cultivation of colonic bacterium that are regarded as beneficial for human health.

While not studied as extensively as FOS, GOS supplementation, in healthy human populations, has also been associated with a significant probiotic effect. Supplementation of 10 grams of GOS throughout a 21 day intervention period showed a significant increase in breath hydrogen accompanied by increases in bifidobacterium ${ }^{84}$. More recently, supplementation of 10 grams of GOS for a 10 day period was found to have significant increase concentrations of colonic microbiota $(\mathrm{P}<0.0001)^{84}$. These results were not seen with longer chain starches and fibers indicating that the low degree of polymerization associated with GOS can more effective prebiotics than longer chain fibers ${ }^{83}$.

Due to poor gastrointestinal tolerance, polyols research regarding a possible prebiotic effect is lacking. Polyols, such as sorbitol, are associated with similar chemical properties as other FODMAPs such as GOS or FOS, thus a possible prebiotic effect associated with colonic fermentation of polyols cannot be ruled out. In vitro analysis has shown that supplementation of sorbitol to bacterial media results in increased bacterial proliferation, most notably increases in lactobacilli, which are largely considered to a beneficial species of colonic microbiota ${ }^{85}$. Furthermore, sorbitol supplementation in rats has been associated with a prebiotic effect as well. Supplementation of sorbitol over a 16-day period in rats resulted in significant 
increases in lactobacillus ${ }^{86}$. While a direct link between polyol consumption and a prebiotic effect in humans has yet to be established experimentally, thus this area of study warrants further research.

Prebiotics and Short-Chain Fatty Acids and Gut peptide hormone production:

Prebiotics are considered to be beneficial to the overall health of the host organism through the ability of these carbohydrates to cultivate the growth of favorable species of colonic microbiota. As previously stated, high levels of bifodobacterium and lactobacilli are associated with health. One of the main hypothesis through which these species of colonic microbiota are able to elicit this beneficial response is through production of short chain fatty acids (SCFA) ${ }^{87,88}$. Short-chain fatty acids are a byproduct of anaerobic metabolism of dietary carbohydrates. Experimental evidence indicates that SCFA production through bacterial fermentation is linked increased serum concentrations of gut peptides such as glucagon- like peptide 1 and peptide $\mathrm{YY}^{89}$. Increases in these peptides have been associated with enhanced insulin sensitivity, increased feelings of satiety, and reduced energy intake ${ }^{43}$.

Glucagon- like peptide 1 (GLP-1) is known to influence a wide variety of metabolic processes throughout the body ${ }^{44}$. GLP-1 is released by L-cells located in the intestine in response to food intake ${ }^{44}$. GLP-1 has shown the ability to confer insulin sensitivity through binding to pancreatic beta cells ${ }^{43}$. Binding of GLP-1 to beta cells increases expression of glucose transporters making the beta-cell more "sensitive" to the presence of extracellular glucose ${ }^{43,44}$. An increase in GLP-1 has been associated increased activity of beta-cell in the presence of glucose ${ }^{43,45}$. 
Furthermore, GLP-1 has also been shown to bind to afferent nerve fibers of the central nervous system resulting in increased feelings of satiety ${ }^{43,45}$. GLP-1 is an important modulator of metabolic homeostasis and has potential to promote decreased energy intake and weight loss 43,45 .

Modulation of colonic microbiota through prebiotic supplementation can have important implications regarding post-prandial circulations of GLP-1. Increases in the SCFA acetate and propionate concentrations have been shown to stimulate circulating concentrations of GLP-1 in mice ${ }^{89}$. Furthermore, increases in GLP-1 were associated with subsequent improvements in glucose tolerance and oxidative stress ${ }^{90}$. Cani et al ${ }^{42}$, utilizing an animal model, examined the physiological effect of FOS when supplemented in to a high fat diet. FOS supplementation was found to increase concentrations of GLP-1 in the proximal colon by $50 \%{ }^{42}$. This increase in GLP-1 was associated with a reduction of energy intake and weight loss at the conclusion of the 15-day intervention ${ }^{42}$. Increases in plasma GLP-1 concentrations have also been associated with increased glucose tolerance ${ }^{18}$. Furthermore, animals lacking the cellular receptor for GLP-1 were associated with hyperglycemia and weight gain despite FOS supplementation ${ }^{18}$. This shows that GLP-1 has an important role regarding postprandial metabolic homeostasis.

Increases in serum concentrations of GLP-1 has also proved to be important in humans. Flint et al. ${ }^{54}$ examined the effect of intravenous infusion of GLP-1 on subjective hunger in a healthy human sample. The experimental group experienced increased feelings of "fullness" and satiety that were not seen in the control group $(p<0.03)^{54}$. Furthermore, increases in GLP-1 was associated with a $12 \%$ decrease in 
energy intake $(p=0.002)$ as well as reductions in blood glucose $(p<0.002)^{54}$ Thus increasing post- prandial GLP-1 concentrations can have important health connotation most notably increased glucose sensitivity, reduced energy intake, and subsequent weight loss ${ }^{18,42}$.

Peptide YY (PYY) is another important gut-derived hormone that is known to influence energy intake and may be stimulated by prebiotics ${ }^{44}$. Like GLP-1, PYY is secreted by L-cells in the small ileum and colon in response to meal intake. PYY has been shown to induce post-prandial satiety ${ }^{55}$ and circulating levels of PYY have been shown to influence size and timing of meals ${ }^{55}$. Furthermore, reduced levels of PYY have been shown to increase incidence of obesity, and restoration of PYY concentration to normal post-prandial levels attenuates appetite ${ }^{55}$. Supplementation of gel-forming fibers has been associated with increases in circulating $\mathrm{PYY}{ }^{91}$. Over a three-week intervention, healthy individuals supplemented PolyGlycopleX, a manufactured soluble dietary fiber. Supplementation of this fiber significantly increased PYY concentrations over the intervention period and was inversely correlated with blood glucose $(\mathrm{r}=-0.27, \mathrm{P}=0.046){ }^{91}$. While this analysis did not show a decrease in weight or energy intake amongst participants, it does provide an alternate mechanism through which supplementation of prebiotics can induce health benefits ${ }^{91}$.

Supplementation of sugar alcohols has also proven effective regarding increasing serum concentrations of PYY ${ }^{92}$. Lactitol supplementation, in male Wister rats, was found to significantly increase postprandial PYY concentrations when compared to the control group and was also associated with subsequent decrease in energy intake ${ }^{92}$. Furthermore, supplementation of lactitol was associated with a 
decrease of colonic $\mathrm{pH}$ through accumulation of SCFA through fermentation of malabsorbed lactitol by colonic microbiota. Supplementation of lactitol in humans significantly increased breath hydrogen production (indicator of colonic fermentation) when compared to the sucrose consuming control ${ }^{92}$. This analysis did not show increased PYY concentrations in humans as a result of lactitol supplementation; however, polyol supplementation did attenuate postprandial decreases in serum PYY concentrations that were seen with the control ${ }^{92}$.

Cani et al. ${ }^{22}$ showed that FOS supplementation amongst a healthy human population can significantly increase breath hydrogen and gut peptide hormone concentrations resulting in a subsequent reduction in subjective hunger ratings. This analysis determined that increased colonic fermentation associated with prebiotic supplementation was associated with significant increases in GLP-1 and PYY concentrations after a standardized test meal ${ }^{22}$. These increases in GLP-1 and PYY were associated with lower subjective hunger ratings as compared to the control group. Furthermore, postprandial GLP-1 concentrations were significantly correlated with breath hydrogen excretion $(r=0.85, p=0.007)$ while postprandial blood glucose concentrations were determined to be inversely correlated with breath hydrogen $(\mathrm{r}=-$ $0.73,-=0.02)^{22}$. This indicates that colonic fermentation of malabsorbed carbohydrates (prebiotics) can significantly increase postprandial concentrations of gut peptide hormones, most notably GLP-1 and PYY, which can translate in to reduced glycemia and hunger ratings amongst a healthy human sample ${ }^{22}$. Glycemic Index, Colonic Fermentation and Blood Glucose: 
Glycemic index (GI) is defined as "blood glucose-raising potential of a standard quantity of carbohydrate, compared with a glucose control" ${ }^{93,94}$. Reduced glycemia associated with a low GI diet is most likely related to the rate at which carbohydrates are absorbed. Carbohydrates with a high glycemic index are easily absorbed and will result in rapid increases in postprandial blood glucose concentrations. Carbohydrates with a low glycemic index are often malabsorbed and thus reduce postprandial glucose levels. Malabsorbed portions of low GI meals are subject to colonic fermentation and been linked to diminished insulin and blood glucose responses which is often accompanied by prolonged periods of satiety as well as reduced subjective hunger ratings ${ }^{13,95}$.

One of the most important health benefits associated with consumption of LGI foods is the ability of these foods to promote "a second meal effect." A concept, originally described by Jenkins et al. ${ }^{95}$ and Wolever et al ${ }^{13}$ states that the glycemic index of an initial meal is a determinant of the postprandial physiological response at subsequent meals ${ }^{13-15,95}$. During a one day test period, healthy volunteers, who were administered a LGI "breakfast" were found to have diminished insulin and post prandial blood glucose responses after administration of a HGI lunch 4 hours later ${ }^{15}$. A follow- up study found a similar relationship between glycemic index and postprandial blood glucose response at subsequent meals ${ }^{14}$. While these studies were not able to establish a relationship between colonic fermentation and improved glucose tolerance, LGI foods containing large amounts of poorly absorbed carbohydrates have the potential to improve glucose tolerance, a concept supported by subsequent research ${ }^{14,15}$. 
A series of studies performed by Nilsson et al ${ }^{16,19,21}$ were able to establish a direct relationship regarding glycemic index, colonic fermentation and subsequent postprandial physiological response. These studies explored whether glycemic index of an evening meal would effect physiological response following a standardized breakfast. Results showed that low GI meals resulted in increases in breath hydrogen when compared to the control $(\mathrm{p}=0.026)$. Furthermore, blood glucose response was reduced following the low GI meal when compared to the control ( $\mathrm{p}=0.019)$. A follow up study by Nilsson et al. ${ }^{21}$ was able to showed that blood glucose response was inversely correlated with breath hydrogen values $(\mathrm{r}=-0.27 ; \mathrm{p}<0.05)$ as well as GLP-1 $(r=-0.26 ; p<0.05)^{39}$. Subjective ratings of satiety were found to be positively correlated with breath hydrogen $(r=-0.27 ; \mathrm{p}<0.01)^{21}$. These studies establish a strong connection between colonic fermentation, thus increased microbial metabolism of poorly digested LGI foods, and improved postprandial responses in a healthy adult population.

More recently, a study was conducted examining the possible second meal effect associated with consuming a meal of brown beans. Brown beans are not only considered to be a low GI food, they are also associated with over 8 grams of soluble fiber, 6 resistant starch, and 3 grams of raffinose (a common galactooligosaccharide) per portion of brown beans ${ }^{20}$. Supplementation of these brown beans, amongst a healthy population, proved to be readily metabolized by colonic microbiota. Breath hydrogen, utilized as an indicator of colonic metabolism, increased $141 \%$ compared to the control $(\mathrm{p}<0.01)^{20}$. These increases in colonic fermentation were also associated with subsequent increases in the short-chain fatty acids proprionate $(16 \% \mathrm{p}<0.05)$ and isobutyrate $(18 \% ; \mathrm{p}<0.01)$. In addition, satiety mediating hormones were increased as 
a result of the brown bean meal (PYY increased 51\%; $<<0.001)$ and post-prandial blood glucose was significantly reduced $(-15 \%$; $<<0.01)$ after a standardized HGI test meal $^{20}$. Finally, subjective hunger ratings were $15 \%$ lower in the experimental group than the control group $(\mathrm{p}<0.05)$. This analysis links colonic fermentation with improvement of important cardiometabolic markers.

Brighenti et al. ${ }^{12}$ examined how colonic fermentation of indigestible carbohydrates supplemented into sponge cake would effect glycemic response at subsequent meals. This analysis utilized a cross-over design in which subjects randomly consumed three types of sponge cake varying in carbohydrate content. The control for this analysis was a high glycemic index sponge cake, containing an easily digested amylopectin starch. The second sponge cake was made with LGI amylose starch. The third meal consisted of a sponge cake containing similar ingredients to the HGI control, however, 5 grams of lactulose was added in place of amylopectin. Lactuose is a synthetically derived disaccharide that is malabosrbed and subsequently fermented by colonic microbiota ${ }^{12}$. Results showed that the LGI and HGI-Lactulose interventions resulted in similar postprandial blood glucose responses when compared to the HGI meal. Improved glucose tolerance was noted in both interventions and significant increases in breath hydrogen were seen $(p<0.001$ for both LGI and HGILaculose). This analysis shows that colonic fermentation of carbohydrates, independent of glycemic index, can improve subsequent glycemic response post meal 12

Colonic Fermentation and Subjective Hunger and Energy Intake: 
The ability of dietary FODMAPs to reduce postprandial insulin and glucose responses is important regarding their ability to induce prolonged periods of satiety ${ }^{96}$. Meals that are high in glycemic index are associated with a sharp increase in blood glucose immediately following meal consumption followed by a drastic decrease in blood glucose 2 hours following the meal ${ }^{96}$. This sharp decline in blood glucose levels has been linked to feelings of hunger and has been seen to immediately precede meal initiation. A meal associated with a low glycemic index does not induce the drastic increases in blood glucose that are seen with HGI meals providing prolonged feelings of satiety. HGI meals were found to increase subjective appetite scores by as much as $44 \%$ amongst a population of obese females ${ }^{96}$. Thus the ability of LGI meals to reduce postprandial glycaemia can have beneficial impact regarding appetite sensations and potentially energy intake.

Studies regarding FODMAPs in an animal model have shown that dietary FODMAPs can have important implications regarding hunger and overall energy intake. Supplementation inulin and $\beta$-glucan in to the diet of mice resulted a $30 \%$ (inulin) and 37\% ( $\beta$-Glucan) reduction in energy intake resulting in a weight reduction amongst the sample ${ }^{97}$. The experimental group experienced increased concentrations of GLP-1, which was attributed to the increases in SCFAs ${ }^{97}$. Consumption of fructans in human populations has also shown to reduce subjective hunger ratings. Peterson et al. ${ }^{23}$ showed that supplementation of fructans in a healthy human population reduces subjective hunger ratings in a dose dependent manner $(\mathrm{p}<0.03)$ under free-living conditions. 
Consumption of dietary FODMAPs has also been shown to mediate energy intake. Rosen et al. ${ }^{24}$ determined that a cereal based breakfast that is high in indigestible carbohydrates were associated with an "increased feeling of fullness" and a "lowered feeling of hunger and desire to eat". Furthermore, this study observed a 16 $\%$ decrease in voluntary energy intake amongst participants after ingesting cereal that is high in indigestible carbohydrates. Colonic fermentation of indigestible carbohydrates was observed through significant increases in breath hydrogen which were found to be inversely correlated with participant "desire to eat" ( $r=-0.24$; $\mathrm{p}=0.053$ ). Breath hydrogen was also inversely correlated with voluntary energy intake at subsequent meals $(\mathrm{r}=-0.34, \mathrm{p}=0.005)$. Colonic fermentation of dietary FODMAPs can have important implications regarding mediation of energy intake and more research is needed to determine if weight loss implications exist for obese individuals.

\section{Colonic Fermentation, SCFA, and Potential to regulate cholesterol synthesis:}

Elevated serum cholesterol levels are considered to be a primary determinant regarding the onset and progression of cardiovascular disease. Total cholesterol levels exceeding $240 \mathrm{mg} / \mathrm{dl}$ have been associated development of cardiovascular disease ${ }^{98}$. The American Heart Association reports that an estimated 13.8\% of the population (31.9 million individuals) meet this criteria ${ }^{98}$. Elevated cholesterol levels, most notably LDL, contribute to endothelial dysfunction in cardiovasculature increasing risk of atherosclerotic development. While effective pharmacological means of lowering cholesterol have been proven effective, dietary interventions have proven advantageous as well. Many dietary interventions center around increases in soluble dietary fibers, which, decrease dietary cholesterol absorption through promotion of 
bile excretion. However, increases in serum short chain fatty acids (SCFA) produced through colonic fermentation of prebiotics have shown the ability to attenuate hepatic cholesterol synthesis through modification of regulatory enzymes, most notably Adenosine 5'- monophosphate activated protein kinase (AMPK) as described in the following sections.

\section{Metabolic Functions of AMPK:}

AMPK is a serine/ threonine kinase and is an important regulatory protein that acts to modulate cellular energy homeostasis ${ }^{99,100}$. Increases in AMPK activity is associated with low cellular energy concentrations (high AMP/ATP ratio). Thus AMPK acts to increase cellular glucose concentrations through activation of GLUT 4 transporters (increasing cellular glucose uptake) and has also been linked to upregulation of cellular glycolysis through activation of 6-phosphofructo- 2 kinase and fructose-2,6-bisphosphatase ${ }^{99,100}$. AMPK helps to maintain cellular homeostasis, in times of energy depletion, through up-regulation of catabolic (energy producing) processes.

AMPK also inhibits anabolic processes (energy consuming pathways) from occurring, most notably hepatic cholesterol synthesis. Research indicates that AMPK has the ability to inhibit cholesterol synthesis in a similar fashion to statins ${ }^{101}$. Cholesterol is synthesized from acetyl-CoA subunits through a complex metabolic pathway in which HMG-CoA reductase catalyzes the rate-limiting reaction ${ }^{102,103}$. HMG-CoA reductase catalyzes the conversion of 3-hydroxy-3-methylglutyl-CoA to mevaloate, a highly endergonic reaction that requires NADH as a cofactor ${ }^{102,103}$. HMG-CoA reductase is subject to enzymatic inhibition through phosphorylation of the 
Serine- 872 residue ${ }^{103}$. This reaction, catalyzed by AMP-activates protein kinase (AMPK) inhibits the binding of the NADH cofactor to the protein, rendering the protein non-functional ${ }^{103}$.

\section{Short Chain Fatty acids and AMPK activity:}

Increases in serum concentrations of SCFA have been associated with increases in the activity of hepatic AMPK ${ }^{102}$. While the mechanism through which SCFAs promote the activity of AMPK has yet to be elucidated, it has been hypothesized that SCFAs increase the AMP/ATP ratio resulting in the subsequent activation of AMPK ${ }^{102}$. Research by Kawaguchi et al. ${ }^{104}$ and Sakabibera et al. ${ }^{105}$ suggest that supplementation of acetate in mice resulted in increased activity of hepatic AMPK. These studies indicated that SCFAs have the ability to directly increase catalytic activity of AMPK ${ }^{104,105}$.

Fushimi et al ${ }^{106}$ showed that supplementation of SCFA can be directly related to decreased rate of cholesterol synthesis. This study examined the effect of supplementation of acetate in a sample of rats. The analysis showed that supplementation of acetate was associated with decreased hepatic concentrations of malonyl- COA indicating inhibition of HMG- COA reductase ${ }^{106}$. This analysis indicates that increases in serum concentrations of SFCA can prove advantageous regarding reduction in cholesterol synthesis.

Short Chain Fatty Acids and Bile synthesis:

An alternate mechanism through which SCFAs can impact serum cholesterol levels is through increased hepatic bile synthesis. Bile acids, synthesized in the liver, 
are excreted after a meal and function to facilitate the digestion and absorption of lipids ${ }^{44,107}$. While bile is composed of a variety of organic compounds, bile acids and free cholesterol are considered to be a main component ${ }^{44}$. Excretion of bile acids is the primary means through which excess cholesterol is removed from the body ${ }^{44}$. Thus, increasing hepatic synthesis of bile will likely result in an increased rate of excretion of free cholesterol. SCFAs have shown the ability to increase bile synthesis through up regulation of cholesterol $7 \alpha$-hydroxylase (CYP7A1) ${ }^{102}$. The CYP7A1 enzyme catalyzes the conversion of cholesterol to $7 \alpha$-hydroxycholesterol which is regarded as a rate limiting step regarding bile acid synthesis ${ }^{44,108}$. The rate of synthesis of bile acids is considered to coincide with the enzymatic activity of CYP7A1 ${ }^{44}$. Studies have shown that mice lacking the CYP7A1 gene are often associated with hyperlipidemia and are also associated with elevated LDL cholesterol 108. Supplementation of SCFAs has shown to increase activity of CYP7A1 and attenuate hyperlipidemia in animal models ${ }^{109}$. Fushimi et al ${ }^{109}$ found that supplementation of acetate in rats increased rate of bile excretion and was found to exert hypolipidemic effects on the experimental sample despite being fed a high cholesterol diet ${ }^{109}$.

\section{Colonic fermentation, SCFAs, and cholesterol synthesis in Humans:}

In the context of a normal human diet, increases in SCFA are achieved through colonic fermentation of prebiotics. Colonic fermentation of carbohydrates, and subsequent increases in SCFA, have been associated with improvements in blood lipids in humans. Brighenti et al. ${ }^{110}$ examined how consuming a breakfast meal, containing 18\% inulin (a known prebiotic) would effect blood lipid values in healthy 
males throughout a 4 week intervention. The analysis showed that supplementation of inulin resulted in statistically significant increase in breath hydrogen, which is a primary indicator regarding the occurrence of colonic fermentation of carbohydrates (test:280 \pm 35 ; placebo $78 \pm 26 \mathrm{ppm}, \mathrm{p}<0.05)^{110}$. These changes in breath hydrogen concentrations were associated with decreases in total cholesterol and triacylglycerols amongst the experimental group ${ }^{110}$. Furthermore, the intervention was determined to be negatively correlated with blood lipid concentrations, and positively correlated with secondary bile acid excretion ${ }^{110}$.

Colonic fermentation of carbohydrates has also proven beneficial amongst a hypercholestererolemic population. Supplementation of 6 grams of $\beta$-glucan per day over a 6-week period resulted in a significant decrease in total cholesterol and LDL cholesterol when compared to the control group ${ }^{52}$. Other studies have shown that soy products containing an added prebiotic resulted in significant reductions in LDL cholesterol $(=0.18 \pm 0.07 \mathrm{mmol} / 1, \mathrm{p}=0.042)$ and improve the $\mathrm{LDL} / \mathrm{HDL}$ ratio $(0.28 \pm 0.11, \mathrm{p}=0.41)^{53}$. While this body of research is incomplete, there is promising evidence regarding the efficacy of increased SCFA through dietary interventions amongst both healthy and at-risk populations.

In conclusion, colonic fermentation of dietary carbohydrates has been linked to a wide variety of health benefits. Most notably, colonic fermentation of dietary carbohydrates has been linked to increases in colonic production of short-chain fatty acids. Increases in SCFA have been subsequently linked to increases in gut peptide hormones, most notably GLP-1 and PYY, resulting in increased insulin sensitivity and reduced post-prandial blood glucose response. Colonic fermentation of fermentable 
carbohydrates has also been linked to prolonged periods of satiety and has been implicated in reducing energy intake. Increases in SCFAs through colonic fermentation have also been associated with decreases in blood lipid concentrations in both healthy and at-risk populations. Most literature regarding this area of study has been conducted through research in animals, and research that has been performed in humans has been subject to high variability. Further research is needed to determine how FODMAPs, when incorporated into a 'free-living diet', can effect colonic fermentation, SCFA concentrations, and subsequent physiological response. 


\section{B. METHODOLOGY}

Overview:

The methodology for this particular study was developed in the Energy Balance Laboratory in the department of Nutrition and Food Sciences, at the University of Rhode Island. Data collection for this study took place during the spring/summer of 2013. This study utilized a randomized, single- blind, crossover design to measure pre- and post-prandial physiological responses of healthy individuals exposed to both high and low FODMAP diets. After baseline data was collected, participants were carefully instructed on the incorporation of high or low FODMAP conditions into their normal, free-living diet. Breath hydrogen, blood glucose, appetite and satiety were assessed in the subject under fasting and postprandial conditions after practicing the diet for a period of three days. Following an 11-day washout period, subjects then underwent above stated laboratory procedures under opposite FODMAP conditions.

\section{Subjects:}

Subjects for this study were recruited from the student population at the University of Rhode Island. They were recruited as a convenience sample through classroom announcements, university e-mail, and flyers. To participate in this study, subjects had to: be healthy (free from chronic diseases such as celiac disease, IBS, diabetes mellitus, or colitis), between the ages of 18-48, not currently following a weight loss diet, no clinical history of functional gut disorders, nonsmoking, non- pregnant, and not taking any antibiotic medications or appetite suppressants. 
Initial Screening/ Assessment:

During visit 1 , individuals reported to the energy balance laboratory and consisted of screening, completion of informed consent, instruction about study protocol, and body composition assessment. Body composition was assessed via BOD POD. Procedure for use of BODPOD is outlined elsewhere. At the completion of visit 1 , subject was scheduled for baseline assessment to occur on a Tuesday.

\section{Baseline Assessment:}

During visits two and four, baseline measurements were performed. Visit 2 was prior to any dietary intervention-taking place. And visit four took place following an 11-day washout period. Participants arrived at the lab following a 10- hour fasting period. Fasting anthropometrics, blood glucose, and breath hydrogen were taken. Subjective appetite was assessed via visual analogue scales (VAS) under fasting conditions. Individuals were then given a high- FODMAP test meal (described below). Blood glucose, breath hydrogen, and appetite questionnaires were reassessed at 30 minutes and 60 minutes post completion of

the test meal. At the conclusion of the laboratory session, subjects, were randomly assigned to follow either the high FODMAP (Diet 2) or low FODMAP (Diet 1) diet for a period of three days.

\section{Post-Intervention Assessment:}

Post- Intervention testing consisted of visits three and five and took place after the subject had been following the dietary intervention for a period of three days. Since baseline assessment took place on a Monday, post-intervention testing took place on Friday. Laboratory protocols, for post-intervention assessment, was 
identical to that of baseline assessment. Subjects received a total of eighty dollars for their participation in the study. Twenty dollars was paid after session three and sixty dollars was paid after the fifth and final visit.

\section{High-FODMAP Test Meal:}

The test meal was designed to mimic real world conditions. The $25 \mathrm{~g}$ of FODMAPS associated with the test meal has been found to induce increases in breath hydrogen concentration indicating carbohydrate malabsorption ${ }^{9}$. The administration of this test meal should be sufficient to observe the postprandial physiological response associated with a high FODMAP conditions in the laboratory setting. The test meal will consist of: 2 pieces of whole grain bread, 1 tsp. of sugar free preserves, $1 \mathrm{tsp}$. of honey, $40 \mathrm{~g}$ of raisins, and $12 \mathrm{oz}$. of milk. This meal consists of $478 \mathrm{kcal}$ and contains $25 \mathrm{~g}$ of FODMAPs per serving (see appendix $\mathrm{F}$ for complete nutritional information). Nutritional information regarding test meal was determined through the use of Nutritional Data System for Research (NDSR) (2012, Nutrition Coordinating Center (NCC), University of Minnesota, Minneapolis, MN).

\section{Educational Material:}

To assist with dietary adherence, each participant received educational material regarding the assigned diet. The educational material came in the form of a booklet that assisted the participant in choosing foods included in their particular diet conditions. The educational material contained "foods to eat" and "foods to avoid" for each food group (see sample in Appendix G). The educational material utilized during this study was created specifically for the purposes of this study utilizing previous research ${ }^{2,33-35}$. Educational materials regarding low FODMAP 
conditions will emphasize the reduction in consumption of fructose, lactose, fructans, and polyols, as these are common in the western diet ${ }^{2}$. For example, the low FODMAP diet stressed utilization of sucrose and glucose as sweeteners instead of high-fructose corn syrup, fructose, isomalt, sorbitol, corn syrup, and honey $^{2}$. Educational materials regarding the high FODMAP diet directly opposed that of the low FODMAP conditions and encouraged the consumption of foods containing these carbohydrates. These educational materials helped participants incorporate high or low FODMAP conditions into their normal, freeliving diet.

\section{Breath Hydrogen:}

Breath hydrogen was analyzed 3 separate times during laboratory sessions 2-5. Fasting values, 30 minute, and 60-minute post meal data will be obtained. Samples were collected with the QuinTron AveoSampler (QuinTron Instrument Co, WI, USA). Collection of sample required that participants inhale maximally, hold breath for a period of 15 seconds, then expel breath into AveoSampler collection device. Experimentally, this method has shown to allow for adequate respiratory exchange to occur resulting in more accurate and reproducible results

30. Once obtained, samples will be immediately analyzed utilizing the QuinTron Microlyzer- CM (Serial number: 106-410-05, QuinTron Instrument Co, WI, USA). The instrument will be calibrated 30 min prior to use, utilizing 98-PPM reference gas (Lot Number: 37083, QuinTron Instrument Co, WI, USA) ${ }^{31,32}$. Samples, at each time point, were collected in duplicate and the results averaged. 
Use of a QuinTron Microlyzer is a validated means through which breath hydrogen can be assessed. QuinTron has been determined to be the gold standard for detecting a positive breath hydrogen test (sensitivity= 0.90 and Specificity= $0.95)^{31}$. Furthermore, QuinTron Microlysers have been used as a means to validate other means of breath hydrogen analysis ${ }^{31}$.

\section{Blood Glucose:}

Blood glucose was analyzed at 3 separate points during each laboratory session. Capillary blood from subjects fingers was utilized for this analysis and was sampled at 0 minutes (Fasting), 30- minutes post-test meal, and 60-munutespost test meal. Protocols for obtaining capillary blood samples were obtained from Alere Inc. Lithium heparin capillary tubes (Lot number: 2221, Alere INC) were utilize to collect $40 \mu \mathrm{L}$ aliquots of capillary blood at each time point. Blood samples were immediately analyzed using a Cholestech LDX (Serial No. SNAA122881, Alere Inc., Waltham MA). Analysis shows that data provided by Cholestech LDX meets accuracy and precision definitions set by the National Cholesterol Education Program ${ }^{111,112}$. These studies demonstrated that the Cholestech LDX is a valid means through which capillary blood samples can be analyzed $^{111,112}$.

\section{Appetite/Satiety:}

Appetite and satiety were assessed using visual analog scales (VAS). During laboratory sessions participants filled out VAS questionnaires regarding satiety, appetite, thirst, and prospective food consumption (see appendix E). VAS has been

determined to be an effective method for assessing appetite and satiety ${ }^{25}$. Flint et 
al. established a direct correlation regarding VAS scores and subsequent energy intake amongst participants ${ }^{25}$.

\section{Anthropometrics:}

All anthropometric measurements will be obtained at the beginning of each laboratory session, after the participant had voided bladder and had undergone a 10-hour overnight fast. Height will be measured to the nearest $0.1 \mathrm{~cm}$ utilizing a stadiometer (Serial No. 110818-002, Quick Medical, Issaquah, WA). Height will be measured two times and averaged together, however, if measurements have a difference of greater than $0.2 \mathrm{~cm}$ then the measurement will be repeated until two measurements are within this range. Weight will be measured in duplicate to the nearest $0.1 \mathrm{~kg}$ using a digital scale (Health-O-Meter, Model 752KL, Northbrook, IL). If there is greater than $0.2 \mathrm{~kg}$ difference, then the measurement will be repeated until two measurements are within this range. Body mass index $\left(\mathrm{kg} / \mathrm{m}^{2}\right)$ will then be calculated utilizing these two measurements. Waist circumference will be measured using a standard tape measure with tensometer. Measurement will be obtained by placing the tape measure around the participant's waist at the level of the umbilicus. If there is greater than $0.5 \mathrm{~cm}$ difference, then the measurement will be repeated until two measurements are within this range. Protocol utilized for collection of anthropometric data has been described elsewhere ${ }^{33}$.

\section{Sample Size:}

Sample size calculations were performed based on differences in blood glucose response seen in a previous study by Nilsson et al ${ }^{39}$. This study found that peak 
blood glucose concentrations decreased $1 \mathrm{mmol} / \mathrm{L}$ ( $18 \mathrm{mg} / \mathrm{dl})$ from control to experimental conditions. Calculations show an effect size of 3.33. All calculations were based on an alpha of 0.05 and power of 0.80 , and were completed using $\mathrm{G}^{*}$ Power (version 3.1.7). According to these calculations, a 8-person sample size would be adequate to achieve sufficient power. Therefore, the proposed sample size of 16 will provide appropriate statistical power for measuring changes in blood glucose response in healthy individuals.

\section{Data Analysis:}

All data was analyzed using SPSS version 21.0 (IBM Corp, Released 2012 Armonk, NY). Skewness and Kurtosis were calculated to determine normality of the data. All data met criteria for normality. Comparison of between diet blood glucose, breath hydrogen, and VAS scores at different time points $(0,30,60 \mathrm{~min})$ were made through the use of a $2 \times 2$ repeated measures analysis of variance. Post hoc t-test were utilized. Analysis of within-treatment effects were examined via paired- t-test for all outcomes. Total Area under the curve was calculated for blood glucose utilizing trapezoid method ${ }^{113}$. Analysis of categorical data was performed utilizing chi-square.

\section{Resources Required:}

Funding for this research came from a grant that was obtained by Dr. Kathleen Melanson from the Research Dietetics Practice Group of the Academy of Nutrition and Dietetics. Laboratory supplies for this study included AveolarSampler test kits, Alere Lipid Profile*GLU cassettes, food for test meal, and a stipend of $\$ 80.00$ that was paid to each subject. Supplies provided by the Department of Nutrition 
and Food Sciences, will include NDSR, QuinTron Microlyser, anthropometric equipment, Cholestech LDX, computers, and software for data analysis. No other university resources were required. 


\section{MACRONUTRIENT CONTENT OF TEST MEAL}

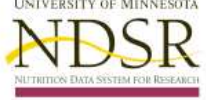

\section{NDSR 2012 Nutrient Totals Report}

Project Abbreviation: PracFODMAP

Participant ID: 002

Date of Intake: 03/14/2013

\section{Primary Energy Sources}

Energy (kilocalories)

Energy (kilojoules)

Total Fat

Total Carbohydrate

Available Carbohydrate

Total Protein

Animal Protein

Vegetable Protein

Alcohol

$\%$ Calories from Fat

$\%$ Calories from Carbohydrate

$\%$ Calories from Protein

$\%$ Calories from Alcohol

$478 \mathrm{kcal}$
$2000 \mathrm{kj}$
$2.131 \mathrm{~g}$
$100.653 \mathrm{~g}$
$94.506 \mathrm{~g}$
$20.018 \mathrm{~g}$
$12.448 \mathrm{~g}$
$7.570 \mathrm{~g}$
$0.000 \mathrm{~g}$
$3.960 \%$
$79.091 \%$
$17.225 \%$
$0.000 \%$

\section{Carbohvdrates}

\section{Total Sugars}

$75.553 \mathrm{~g}$

Fructose

$28.494 \mathrm{~g}$

Galactose

$0.661 \mathrm{~g}$

Glucose

Lactose

$24.564 \mathrm{~g}$

Maltose

$18.234 \mathrm{~g}$

$1.864 \mathrm{~g}$

$0.702 \mathrm{~g}$

Sucrose

$11.650 \mathrm{~g}$

Starch

$19.708 \mathrm{~g}$

Added Sugars (by Total Sugars)

$20.526 \mathrm{~g}$

\section{Fiber}

Total Dietary Fiber

$6.146 \mathrm{~g}$

Soluble Dietary Fiber

$1.650 \mathrm{~g}$

Insoluble Dietary Fiber

$4.462 \mathrm{~g}$

Pectins

$0.762 \mathrm{~g}$

\section{Sugar Alcohols (polvols)}

Erythritol

Inositol

Isomalt

Lactitol

Maltitol

Mannitol

Pinitol

Sorbitol

Xylitol

Note: DSAM nutrients are not included in these totals. Nutrient totals may not equal the sum of their parts. (Refer to the NDSR User Manual.

This record contains incomplete information. 


\section{Breath Hydrogen Collection Protocol}

\section{Warm Up Period}

1. Turn on system 15 minutes (at least) prior to use.

2. Following the warm up period, adjust the front panel labeled "parts per million" until it reads " 000 "

\section{Calibration}

Materials needed-Reference gas, SivRite cartridge, syringe, stopcock

1. Pull "out" valve stem so that pilot light turns GREEN

2. Using a syringe with stopcock, extract $20 \mathrm{ml}$ of reference gas (concentration of $98 \mathrm{ppm})$.

3. Place the SivRite cartridge directly into the flush port.

4. Inject the reference gas into the machine via the SivRite cartridge. (if reference gas cannot be injected, check to make sure A-the valve stem is pulled all the way out and B- the stopcock is open.)

5. After the gas has been flushed, push the valve stem "in" until the GREEN light changes to RED and observe the meter response.

6. Once the meter response becomes stable, adjust the "calibrate" knob until it reads "098".

7. Pull the valve stem "out" and the meter response should read "000".

8. If meter response does not read "000", re-zero the instrument and repeat calibration process.

9. Continue process until instrument is properly calibrated

\section{Collection/ Analysis of Sample:}

Materials needed-Breath Collection kit (including mouth piece, collection bag and discard bag), SivRite cartridge, syringe, stopcock

1. Ask subject to take deep breath and hold breath for 15 seconds.

2. After 15 second has passed, have subject exhale normally

3. As individual is exhaling, withdraw $20 \mathrm{~mL}$ of expelled air via the syringe with stopcock.

4. Pull the valve stem "out" so that the pilot light turns GREEN

5. Inject $20 \mathrm{ml}$ of the sample gas into the machine via the SivRite Cartridge

6. Push valve stem "in" until the light turns RED

7. Record the $\mathrm{H} 2$ concentration ( $\mathrm{ppm}$ ) presented in the meter response

8. Pull Valve stem "out" so that light turns GREEN and release the sample from the port

9. Using the syringe, back flush $40 \mathrm{ml}$ of room air into the machine.

10. Repeat analysis using an additional $20 \mathrm{ml}$ taken from the original collection bag.

11. Take the average of the two numbers

This process can be done after the participant has left. Samples are good for 23 hours in the breath collection bag 


\title{
E. IN-LAB DATA COLLECTION SHEETS
}

NAME

\author{
The Carb Study \\ Participant Screening Form
}

Inclusion Criteria:

YES NO Healthy, not experiencing symptoms of any illness such as a cold or flu

YES NO Not currently on a weight loss diet

Exclusion Criteria:

YES NO Food Allergy

If Yes

YES NO Hypolactasia (Lactose Intolerance)

YES NO Celiac Disease

YES NO Gluten Intolerance

YES NO Current smoker

YES NO Irritable Bowel Syndrome

YES NO Pregnant or lactating

YES NO Type 1 or Type 2 Diabetes Mellitus

YES NO Diverticular Disease

YES NO Colitis such as Crohn's disease or Ulcerative Colitis

YES NO Adrenal disease

YES NO Hypoglycemia (low blood sugar)

YES NO Seizures

YES NO Kidney or bladder problems

YES NO Stomach ulcers

YES NO Thyroid diseases

YES NO Currently taking appetite suppressant medication 


\begin{tabular}{|c|c|c|c|}
\hline Subject \#/ ID & Condition ID & Visit \# & Date \\
\hline & & 1 & \\
\hline
\end{tabular}

Investigators Present:

\section{Initial Assessment}

Screening Tool and Consent Form

Eligibility Verified using screening tool Informed consent explained and both forms signed by subject

\& investigator.

\section{BodPod}

Body fat percentage as measured by BodPod. Record measurements to one decimal place $(.0 \%) \_\%$

Demographics

Age:

Gender:

Contact Information/Preferred Means of Contact

_ Email:

Cell phone:

- Text

\section{Visit 2 Scheduling}

Inform subject that they must refrain from eating or drinking anything except water before reporting to the lab for their next visit

Visit 2 must be scheduled on a Tuesday and after a 10 hour fast. That being said, the date of the participant's next appointment will be:

$$
\text { Tuesday, }
$$
at A.M. 


\begin{tabular}{|c|c|c|c|}
\hline Subject \#/ ID & Diet \# & Visit \# & Date \\
\hline & & 2 & \\
\hline
\end{tabular}

Investigator/s Present:

\section{PREIntervention}

Time:

10 hour fast verified.

Subject voided bladder.

\section{Fasting Values}

Height (cm) Height $(\mathrm{cm})$ Ave. $/ 100=$ $\mathrm{m}$ $-$ $\mathrm{m}^{2}=$

$\overline{\text { Weight (lb) }}$ Weight (lb) Ave. $12.2=$

BMI $\left(\mathrm{kg} / \mathrm{m}^{2}\right)$ $\mathrm{kg}$

$\mathrm{WC}(\mathrm{cm})$ WC $(\mathrm{cm})$

Ave. $\mathrm{cm}$ Give Appetite Questionnaire \#1

Breath Hydrogen Concentration Results:

$\mathrm{BH} 2$ ppm BH2 ppm Ave H2. ppm Capillary Glucose Concentration Result: $\mathrm{mg} / \mathrm{dL}$.

\section{Test Meal}

Give prepared Test Breakfast and record the following times:

Start Time

Meal completion:

30 minutes post meal completion time:

Start Timer

1 hour post meal completion time:

Tell subject that they must remain in the lab for 1 hours with no food or drink.

24-hour recall

\section{0 minutes after meal completion time}

Appetite Questionnaire \#2

Breath Hydrogen Concentration Results:

$\mathrm{BH} 2 \_\mathrm{ppm} \quad \mathrm{BH} 2$

Capillary Glucose Concentration Result: ppm Ave $\mathrm{H} 2$. $\mathrm{mg} / \mathrm{dL}$. ppm

Explain and give subject information regarding assigned Diet \# 


\section{1 hours after meal completion time}

Give Appetite questionnaire \#3

Breath Hydrogen Concentration Results:

$\mathrm{BH} 2 \_\mathrm{ppm} \quad \mathrm{BH} 2$

ppm Ave H2. Capillary Glucose Concentration Result:___ mg/dL.

ppm

\section{Visit 3 Scheduling}

Inform subject that they must refrain from eating or drinking anything except water before reporting to the lab for their next visit

Visit 3 must be scheduled on Friday and after a 10 hour fast. That being said, the date of the participant's next appointment will be:

Friday, at A.M.

Describe any unusual events:

- about the subject: was sick, didn't follow directions, didn't like test meal, remarks/questions about the study. Equipment malfunctions 


\begin{tabular}{|c|c|c|c|}
\hline Subject \#/ ID & Diet \# & Visit \# & Date \\
\hline & & $\mathbf{3}$ & \\
\hline
\end{tabular}

Investigator/s Present:

\section{POST-Intervention}

Time:

10 hour fast verified.

Subject voided bladder.

\section{Fasting Values}

Height (cm) Height $(\mathrm{cm})$ Ave.

$/ 100=$ $\mathrm{m}$

${ }^{-}$

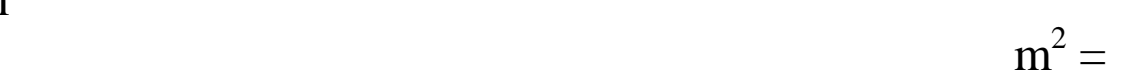

Weight (lb)

Weight (lb)

Ave.

$12.2=$

BMI $\left(\mathrm{kg} / \mathrm{m}^{2}\right)$ $\mathrm{kg}$

$\mathrm{WC}(\mathrm{cm})$

$\mathrm{WC}(\mathrm{cm})$

Ave. $\mathrm{cm}$ Give Appetite questionnaire \#1

Breath Hydrogen Concentration Results:

$\mathrm{BH} 2$ ppm BH2 ppm Ave $\mathrm{H} 2$. ppm Capillary Glucose Concentration Result: $\mathrm{mg} / \mathrm{dL}$

\section{Test Meal}

Give prepared Test Breakfast and record the following times:

Start time:

Meal completion:

30 minutes post meal completion time:

1 hours post meal completion time:

Start timer

Tell subject that they must remain in the lab for 1 hours with no food or drink.

24-hour recall

30 minutes after meal completion time

Give Appetite Questionnaire \#2

Breath Hydrogen Concentration Results:

$\mathrm{BH} 2 \_\mathrm{ppm} \quad \mathrm{BH} 2$ ppm Ave H2. ppm Capillary Glucose Concentration Result: $\mathrm{mg} / \mathrm{dL}$.

1 hours after meal completion time 
Give Appetite Questionnaire \#3

Breath Hydrogen Concentration Results:

$\mathrm{BH} 2 \_\mathrm{ppm} \quad \mathrm{BH} 2$

Capillary Glucose Concentration Result:

ppm Ave H2.

ppm

Subject received $\$ 20$.

\section{Visit 4 Scheduling}

Inform subject that they will now have an eleven-day washout period where they should not follow any specific diet (eat what they usually eat)

Inform subjects that they must refrain from eating or drinking anything except water before reporting to the lab for their next visit

Visit 4 must be scheduled eleven days from now on Tuesday and after a 10 hour fast. That being said, the date of the participant's next appointment will be:

$$
\text { Tuesday, }
$$

at

A.M.

Describe any unusual events:

- about the subject: was sick, didn't follow directions, didn't like test meal, remarks/questions about the study

- equipment malfunctions 


\section{F. QUESTIONNAIRES}

Energy Balance Lab Satiety Rating Scale

\begin{tabular}{|l|l|l|l|}
\hline Subject \#/ ID & Condition ID & Visit \# & Date \\
\hline & & & \\
\hline
\end{tabular}

Clock Time: (0)

1. How hungry are you right now?

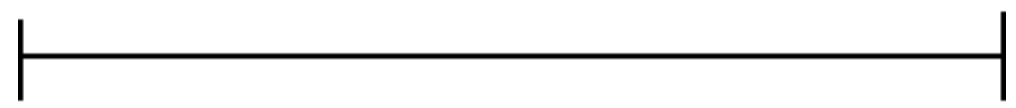

Not at all

Extremely

2. How satisfied (satiated) are you right now?

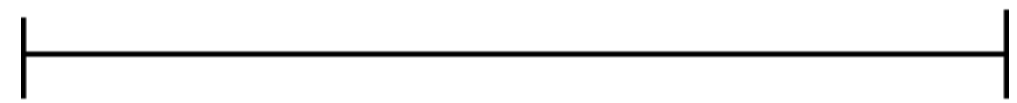

Not at all

Extremely

3. How much could you eat right now?

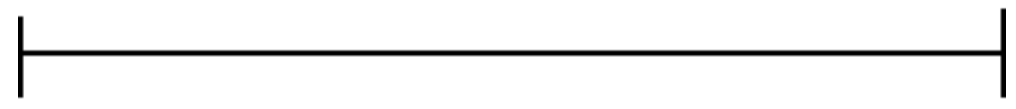

Nothing

Vast Quantities

4. How thirsty are you right now?

Not at all

Extremely

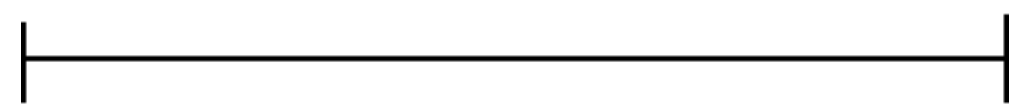

5. A) Are you currently suffering from any abdominal discomfort? Yes No

B) If yes, how severe is the abdominal discomfort?

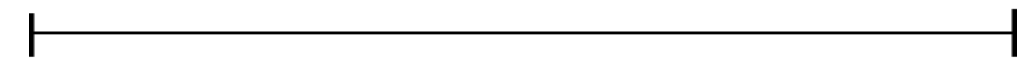

Discomfort

No pain

Severe 
Energy Balance Lab Satiety Rating Scale

\begin{tabular}{|l|l|l|l|}
\hline Subject \#/ ID & Diet & Visit \# & Date \\
\hline & & & \\
\hline
\end{tabular}

Clock Time: (30)

1. How hungry are you right now?

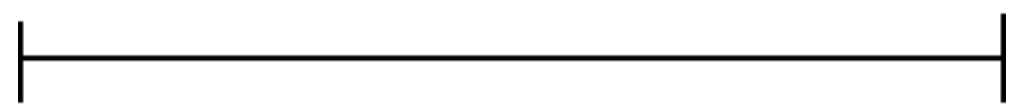

Not at all

Extremely

4. How satisfied (satiated) are you right now?

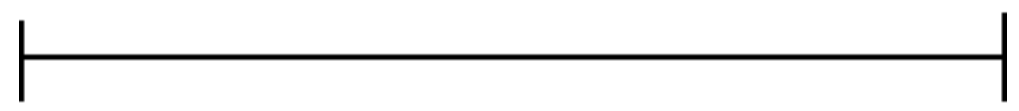

Not at all

Extremely

5. How much could you eat right now?

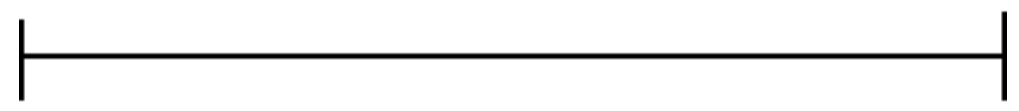

Nothing

Vast Quantities

4. How thirsty are you right now?

Not at all

Extremely

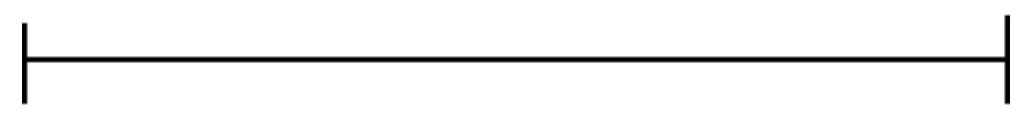

5. A) Are you currently suffering from any abdominal discomfort? Yes No

B) If yes, how severe is the abdominal pain?

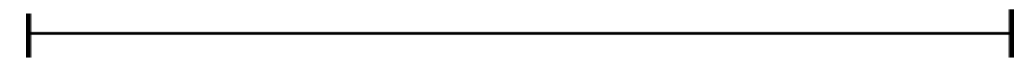

Do pain
Energy Balance Lab Satiety Rating Scale 


\begin{tabular}{|l|l|l|l|}
\hline Subject \#/ ID & Diet & Visit \# & Date \\
\hline & & & \\
\hline
\end{tabular}

Clock Time: (60)

1. How hungry are you right now?

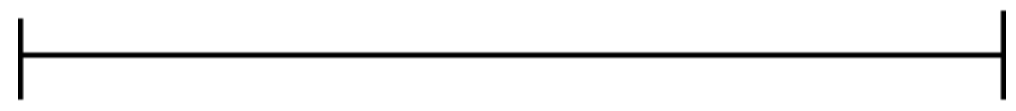

Not at all

Extremely

6. How satisfied (satiated) are you right now?

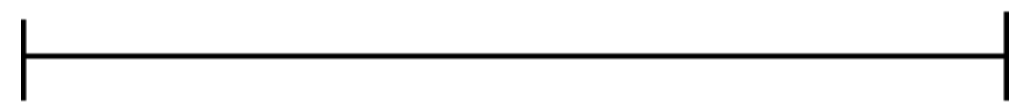

Not at all

Extremely

7. How much could you eat right now?

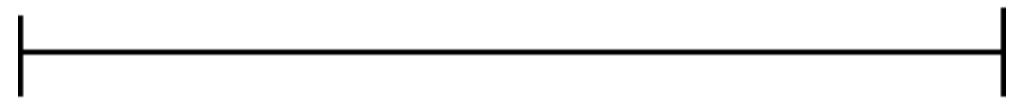

Nothing

Vast Quantities

4. How thirsty are you right now?

Not at all

Extremely

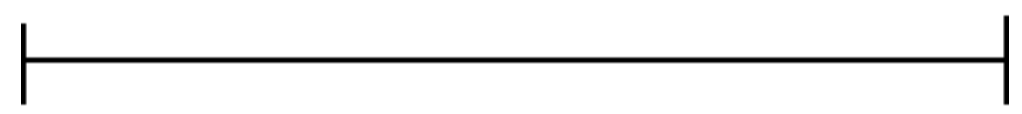

5. A) Are you currently suffering from any abdominal discomfort? Yes No B) If yes, how severe is the abdominal pain?

Discomfort

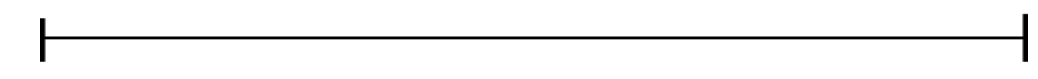

No pain

Severe 


\section{G. DIETARY INSTRUCTION BOOKLETS}

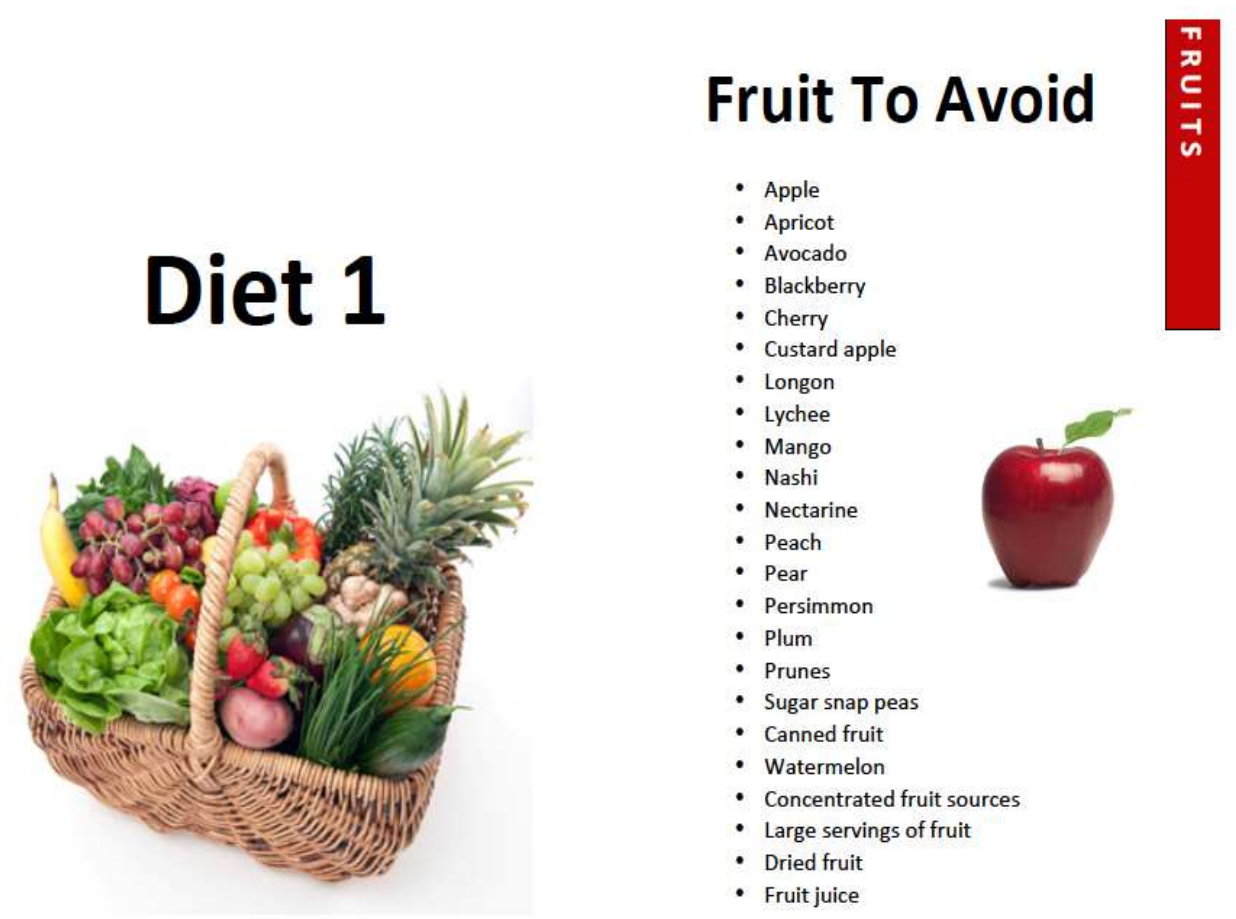




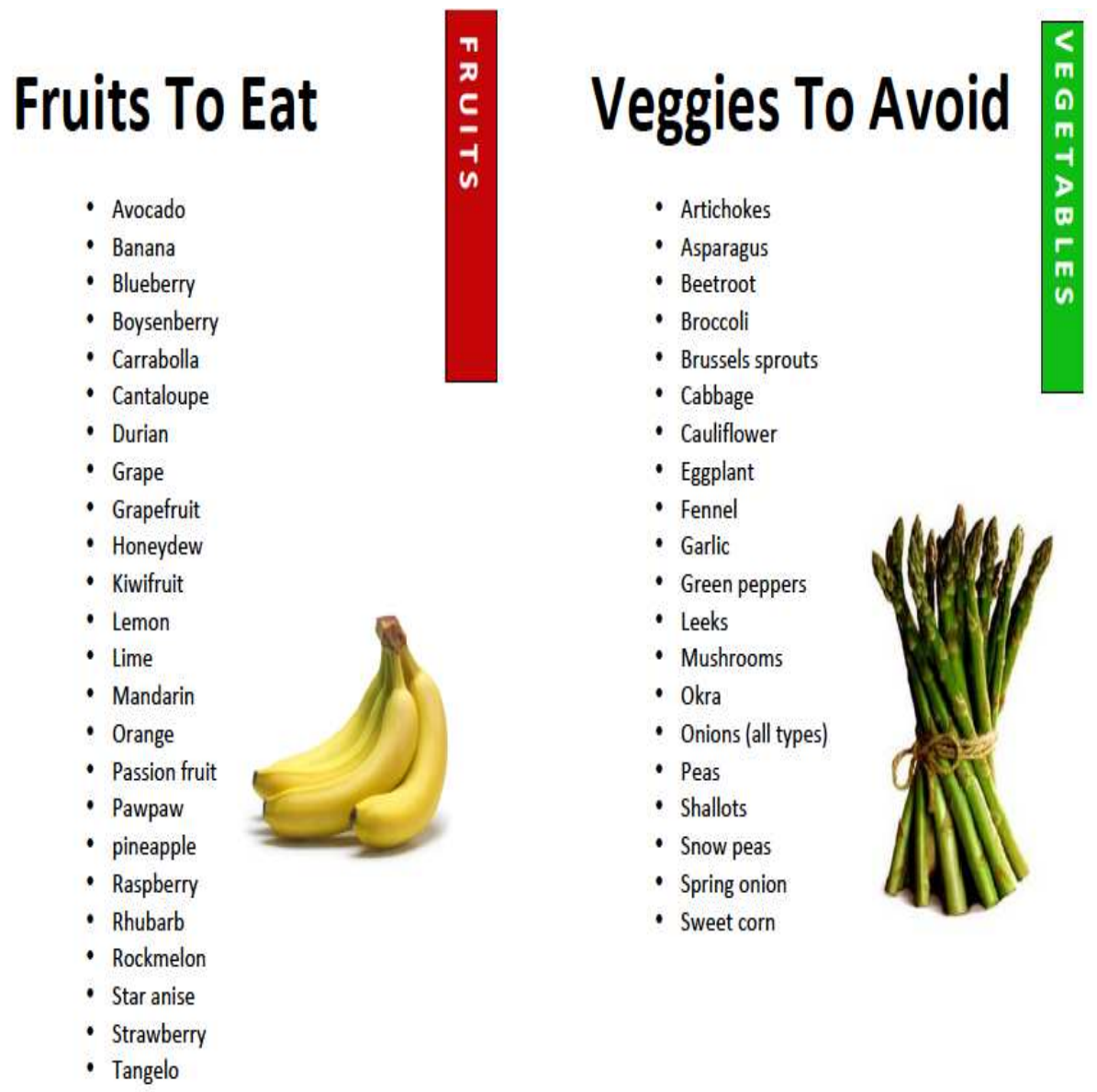




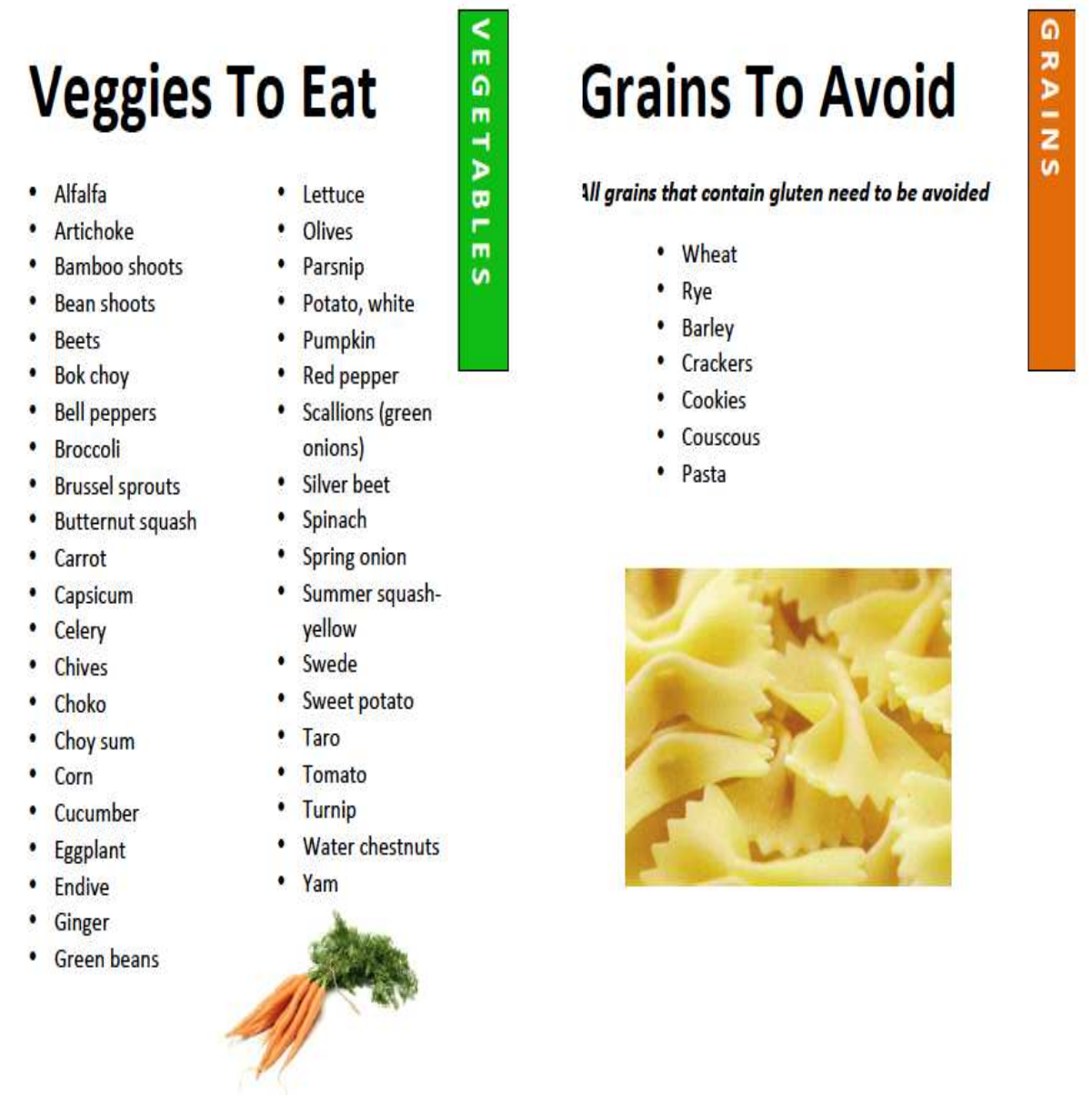




\section{Grains To Eat}

All gluten-free grains are okay

- Gluten free bread

- Gluten free pasta

- Arrowroot

- Corn flakes

- Millet

- Oats

- Oat bran

- Polenta

- Phylum

- Quinoa

- Rice: brown and white

- Rice bran

- Sorghum

- Tapioca

\section{Dairy To Avoid}

- Soft un-ripened cheeses

- Cottage

- Cream

- Mascarpone

- Ricotta

- Milk from cows, goats or sheep

1. Yogurt

- Custard

- Ice cream
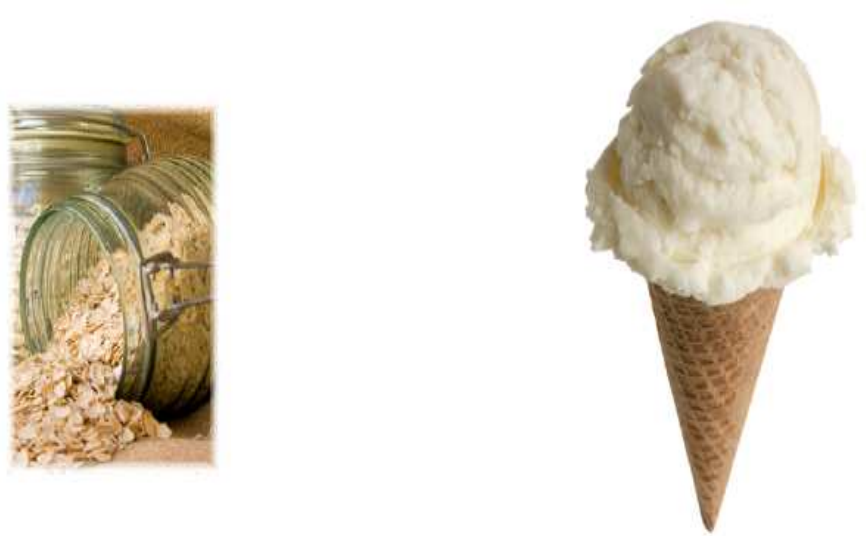


\section{Dairy To Eat}

0

- Hard cheeses
- Cheddar
- Swiss
- Parmesan
- Brie
- Feta
- Camembert
- Mozzarella

- Lactose-free Milk

- Lactose-free Yogurt

- Ice cream Substitutes

- Galati

- Sorbet

- Butter

- Butter substitute

$$
\text { - Olive oil }
$$

- Almond milk

- Coconut Milk

- Oat Milk

- Rice Milk

- Soy milk

- Kefir

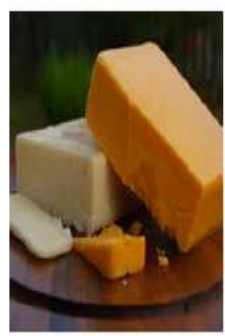

\section{Protein To Avoid}

3readed Meat

'istachios

tII Beans and Legumes

- Baked beans

- Black Beans

- Chickpeas (Garbanzo beans)

- Hummus

- Kidney beans

- Lentils

- Red kidney beans

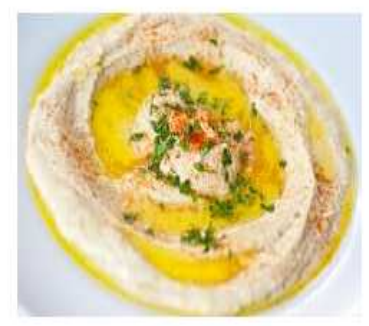



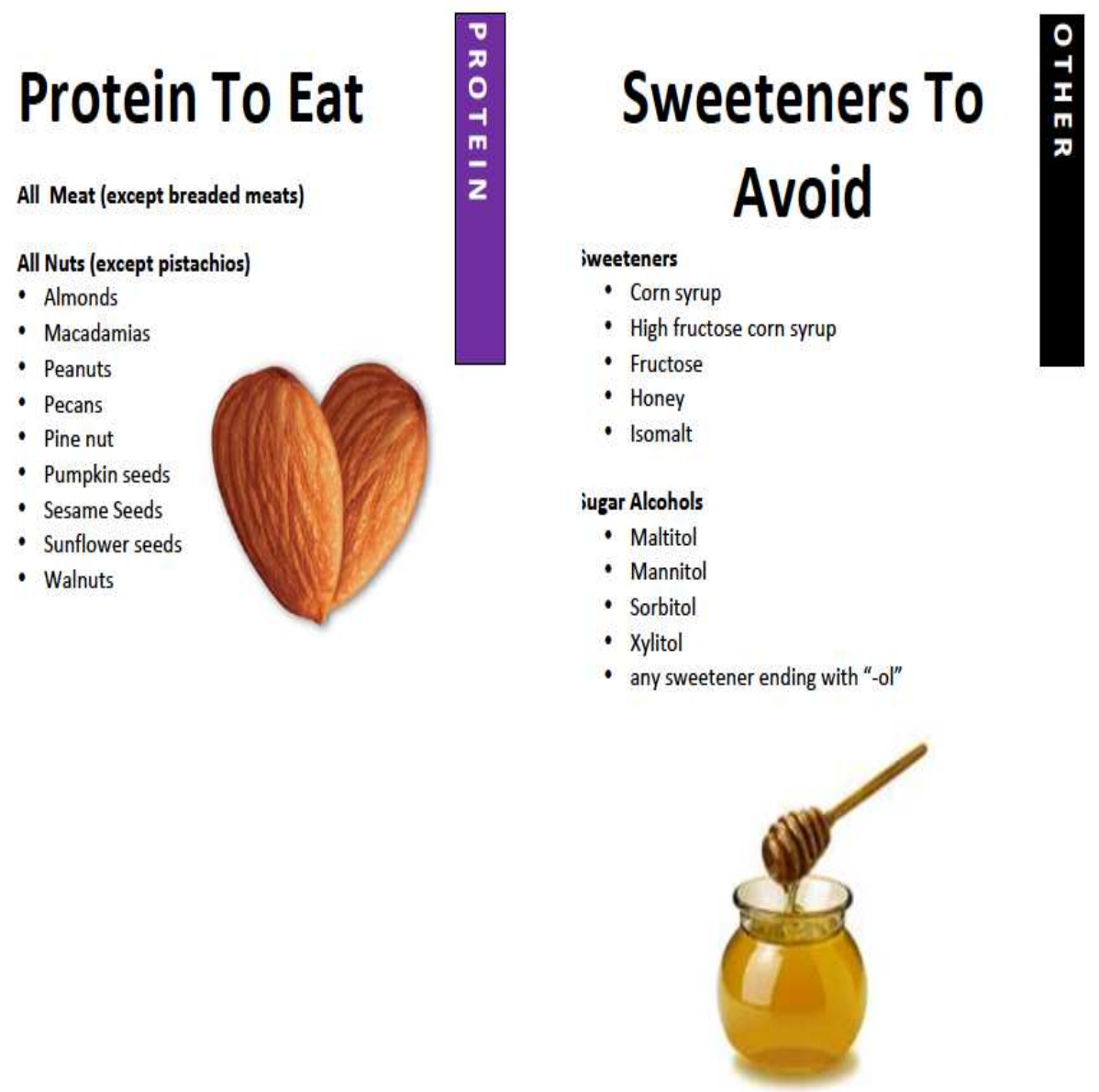


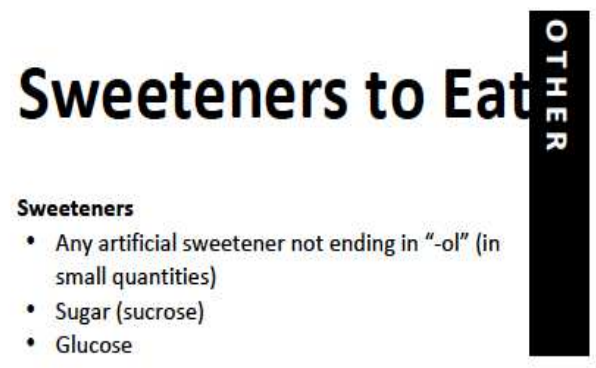

Honey substitutes

- Maple syrup

- Golden syrup

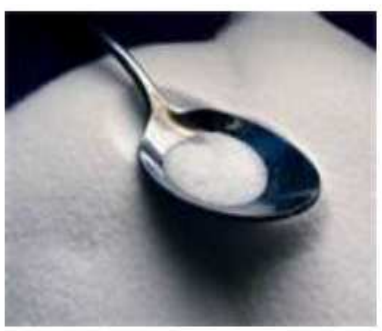

\section{Diet Tips}

- "Gluten-free" breads, pastas, and bake mixes are good alternatives to the usual kinds, which contain wheat.

- Even for "Allowed" foods, try not to eat too much in one sitting, but also don't stress over exact serving sizes.

- For example, you shouldn't eat a whole two-pound bag of grapes in one day, but don't feel like you need to count out exactly how many grapes you can have.

- Check the Ingredients List on food labels and look for any prohibited foods.

- If you're not sure if an item at a restaurant contains a particular prohibited food, ask your server or someone who works there if they have that information.

- This will be especially useful for foods containing wheat and milk.

- It may be easier to avoid or limit use of restaurants for the duration of the test diet.

\section{Alcohol}

dllowed

- Wine (limit one 5 oz serving)

- Beer (limit one 12 oz serving)

- Neutral Grain Spirits (limit one 1.5 oz serving) - Ex. vodka, whiskey, gin

Not Allowed

- Rum

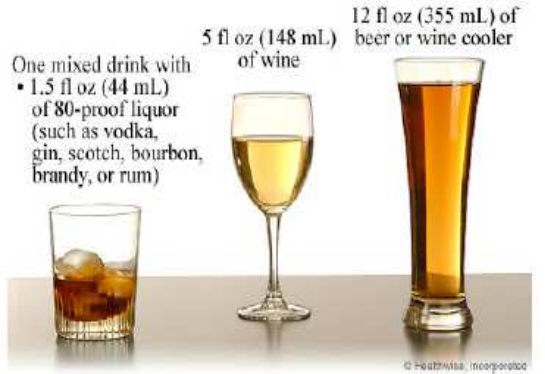

\section{Contact Information}

Jnsure about something?

$\mathrm{n}$ a pinch it is probably safer to avoid it sut feel free to call us at, Lab Phone 401374-2067, or e-mail us at :arbstudy.URI@gmail.com during the day f you have any questions. 


\section{Discouraged Fruits}

\section{Diet 2}

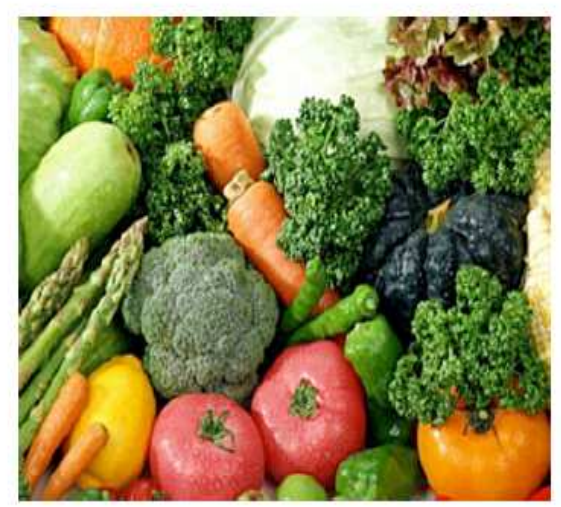

- Avocado

- Banana

- Blueberry

- Boysenberry

- Carrabolla

- Cantaloupe

- Durian

- Grape

- Grapefruit

- Honeydew

- Kiwifruit

- Lemon

- Lime

- Mandarin

- Orange

- Passion fruit

- Pawpaw

- Pineapple

- Raspberry

- Rhubarb

- Rockmelon

- Star anise

- Strawberry

- Tangelo

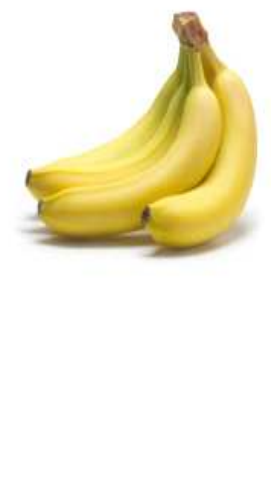




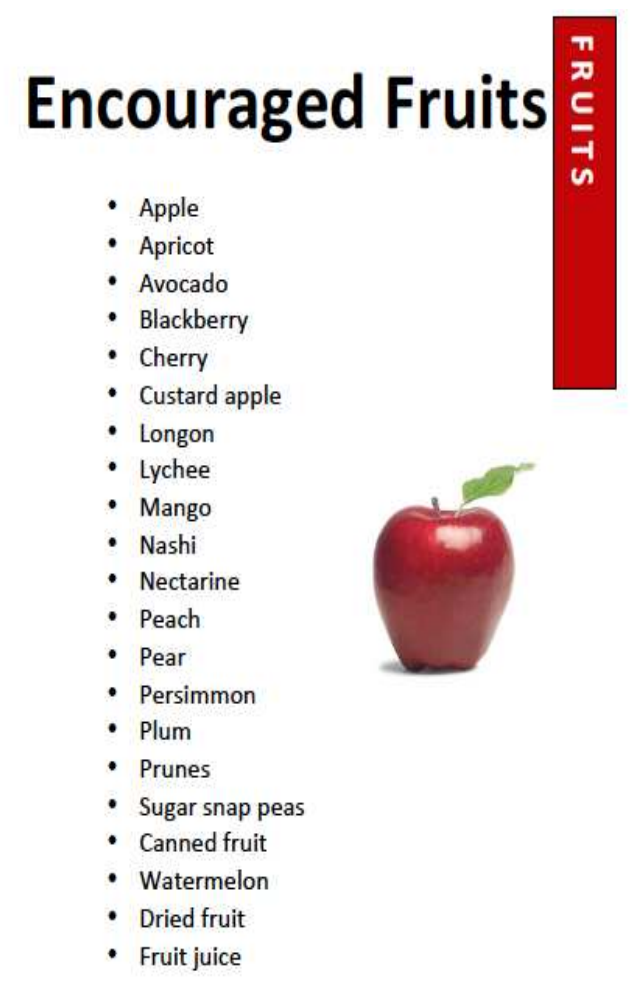

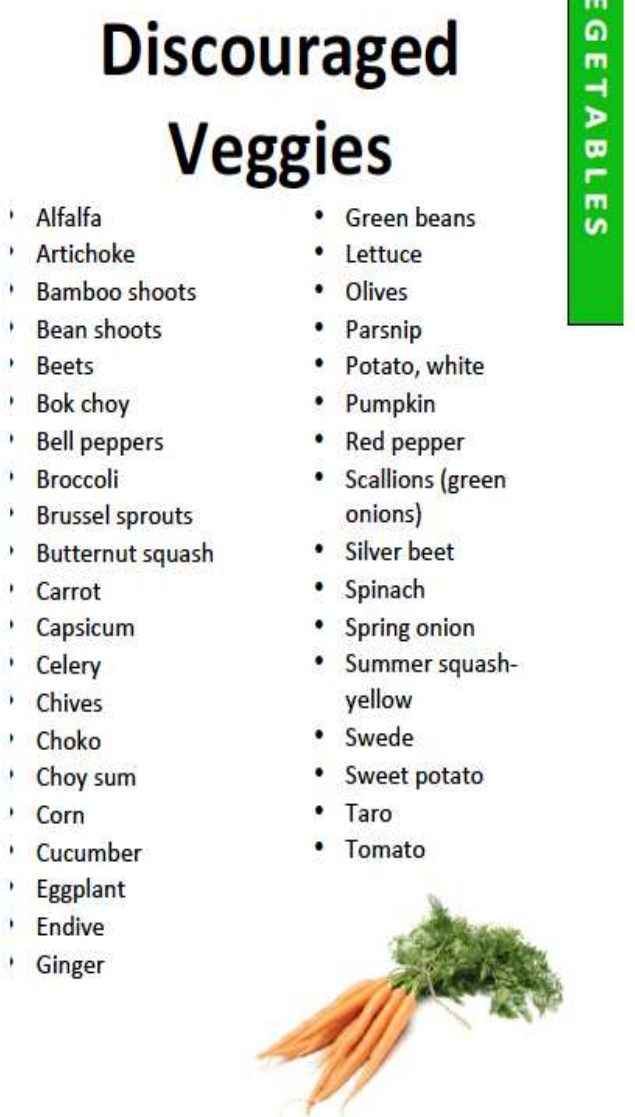




\section{Encouraged Veggies}

- Artichokes

- Asparagus

- Beetroot

- Broccoli

- Brussels sprouts

- Cabbage

- Cauliflower

- Eggplant

- Fennel

- Garlic

- Green peppers

- Leeks

- Mushrooms

- Okra

- Onions (all types)

- peas

- Shallots

- Snow peas

- Spring onion

- Sweet corn

- Yam

- Zucchini

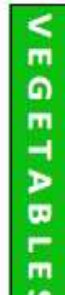

Discouraged Grains

- Arrowroot

- Corn flakes

- Millet

- Oats

- Oat bran

- Polenta

1. Phylum

- Quinoa

- Rice: brown and white

- Rice bran

- Sorghum

- Tapioca

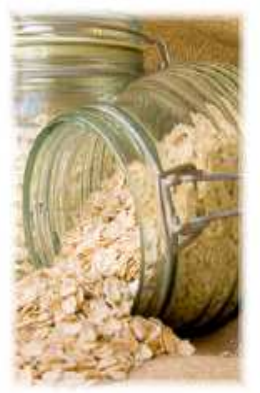

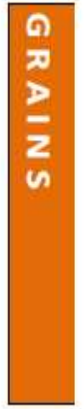

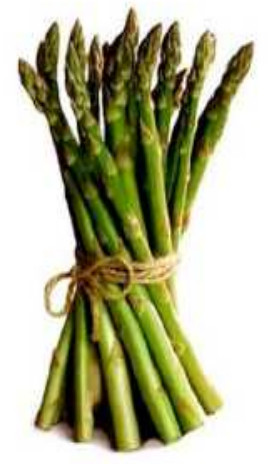



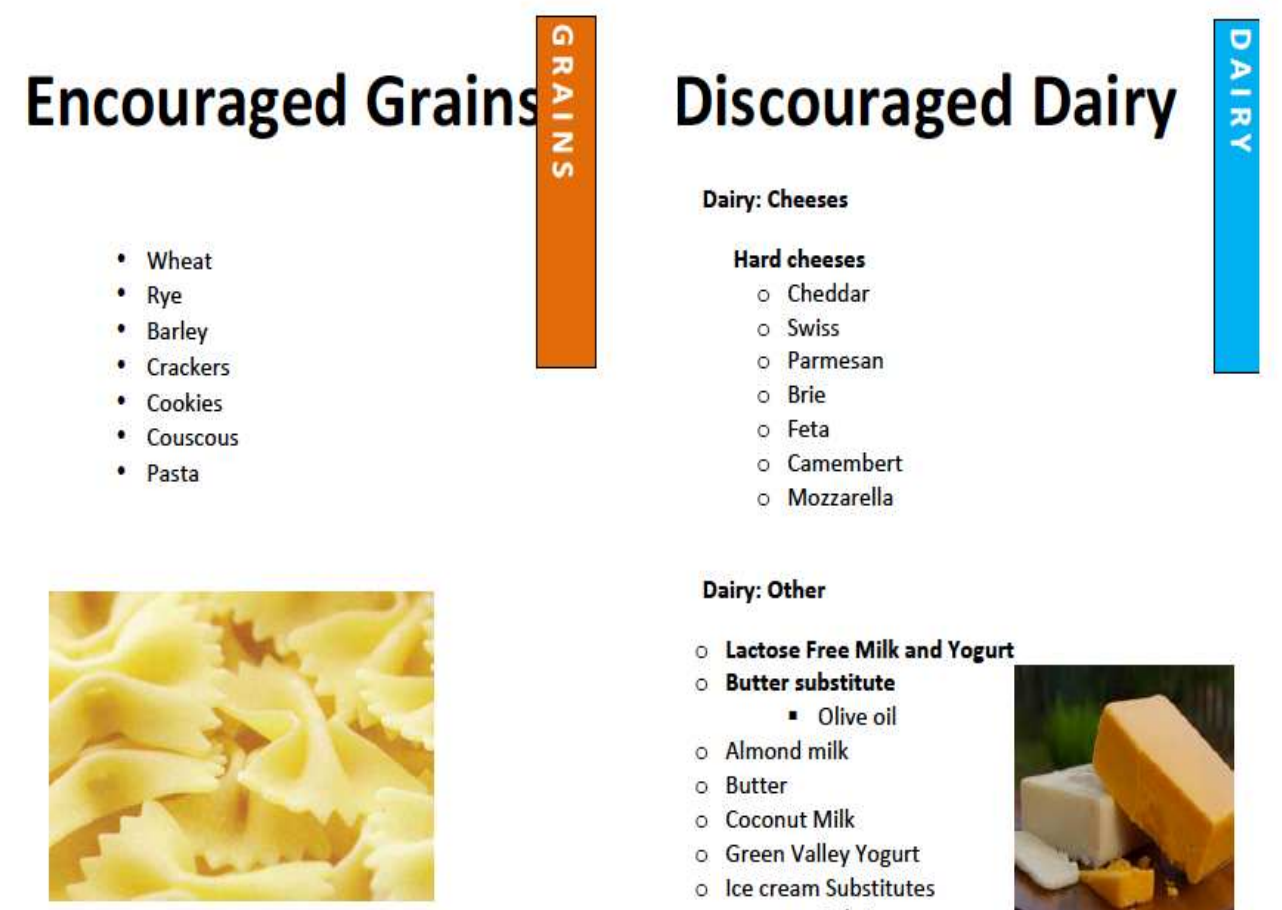

Dairy: Other

- Lactose Free Milk and Yogurt

- Butter substitute

- Olive oil

- Almond milk

- Butter

- Coconut Milk

- Green Valley Yogurt

- Ice cream Substitutes - Galati

- Sorbet

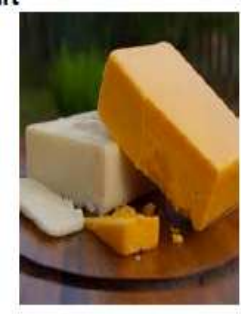

- Lifeway Kefir (strawberry or blueberry)

- Oat Milk

- Rice Milk

- Soy milk 


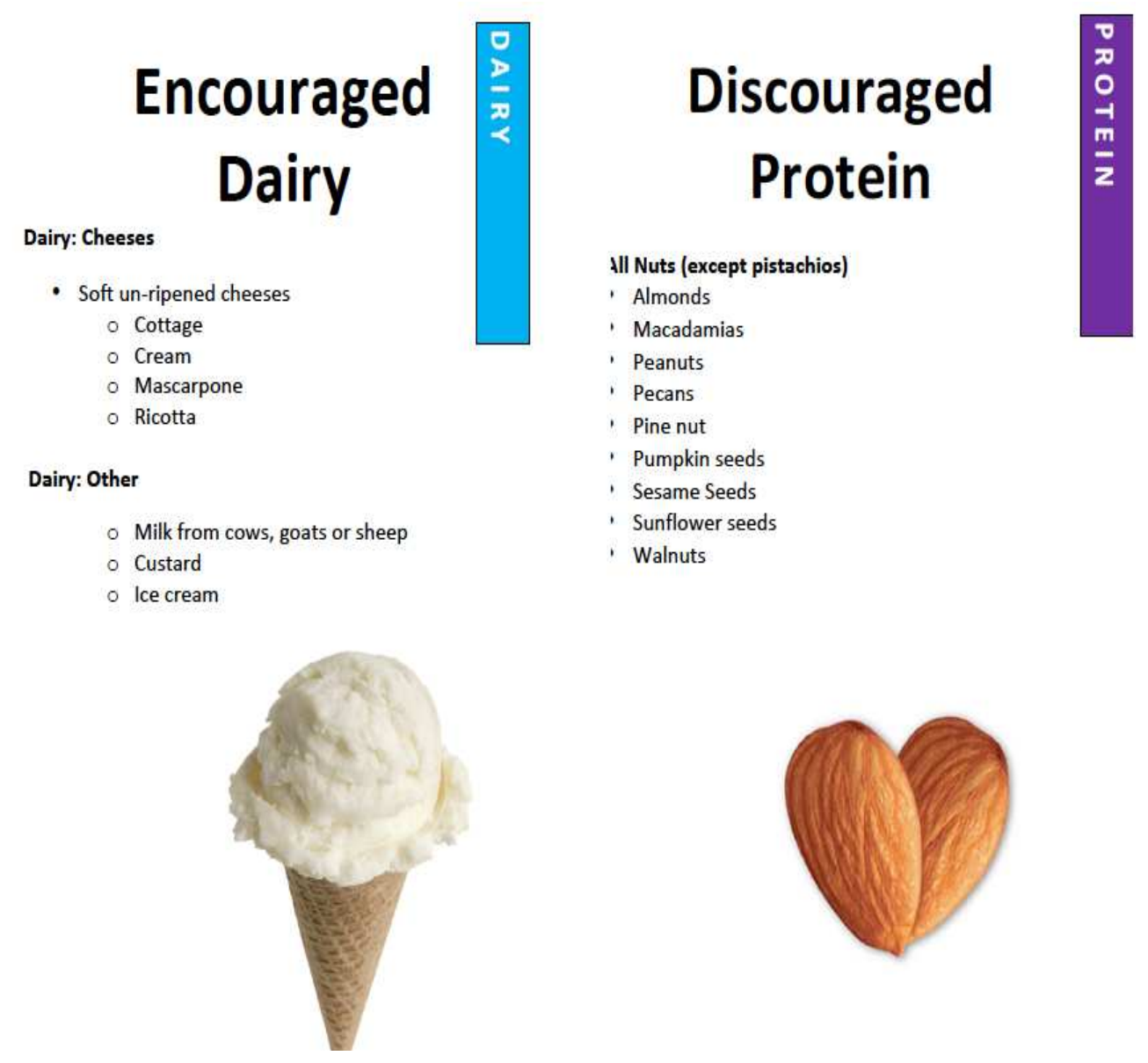




\section{Encouraged Protein}

Nuts

- Pistachios

All Legumes

- Baked beans

- Chickpeas

- Hummus

- Kidney beans

- Lentils

- Red kidney beans

- Black Beans

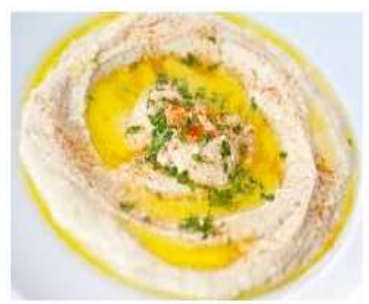

Discouraged Sweeteners

iweeteners

- Any artificial sweetener not ending in "-ol" (in small quantities)

- Sugar (sucrose)

- Glucose

toneys substitutes

- Maple syrup

- Golden syrup

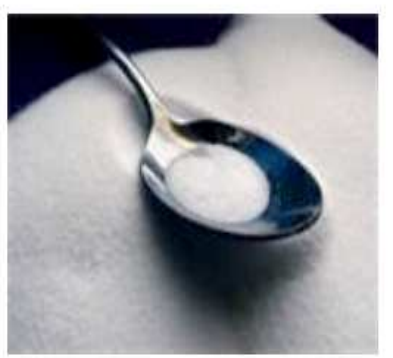




\title{
Encouraged Sweeteners
}

\author{
Sweeteners \\ - Corn syrup \\ - High fructose corn syrup \\ - Fructose \\ - Honey \\ - Isomalt \\ Sugar Alcohols \\ - Maltitol \\ - Mannitol \\ - Sorbitol \\ - Xylitol \\ - any sweetener ending with "-ol"
}

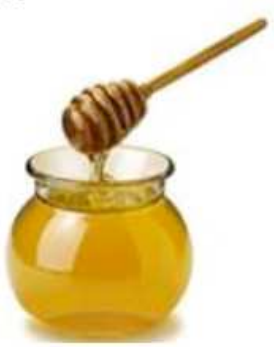

\section{Diet Tips}

- Aim to have at least 2 "Encouraged" foods at every meal and at least 1 "Encouraged" food for every snack. More is better, if you can!

- Try not to have "Discouraged" foods, in favor of "Encouraged" foods. To this end, it might be prudent to limit yourself to 1 "Discouraged" food per meal.

- You can eat any foods NOT in this booklet as you normally would. 


\title{
Contact Information
}

\author{
Unsure about something? \\ Feel free to call us at, Lab Phone 401- \\ 874-2067, or e-mail us at \\ carbstudy.URI@gmail.com during the day \\ if you have any questions.
}




\section{H. Consent Form for Research}

The University of Rhode Island

Department of Nutrition and Food Sciences

Kingston, RI 02881

The Carb Study

\section{CONSENT FORM FOR RESEARCH}

You have been invited to take part in a research project described below. The researcher will explain the project to you in detail. You should feel free to ask questions. If you have more questions later, Kathleen Melanson, the person mainly responsible for this study, will discuss them with you (Energy Balance Lab, 310 Ranger Hall, 401-874-2067). You must be at least 18 years old to be in this research project. You will also need to be available for two Tuesday and two Friday mornings for testing.

Description of the project:

You have been asked to participate in a research study designed to test the acceptability of and physiological responses to the manipulation of dietary carbohydrate sources.

\section{What will be done:}

Following an initial assessment visit, all study volunteers will follow two specific diets for three days each, with 11 days of your normal eating inbetween:

- You would follow the first diet for three days between the second lab and third lab visits.

- During the 11-day period between the third and fourth lab visits, you would not be asked to follow any specific diet; you would just return to your normal diet.

- You would follow the second diet for three days between the fourth and end the fifth lab visits, which marks the end of the study.

Total time in the lab will amount to about 8.5 hours: 30 minutes for first visit, and about two hours each for the other four visits. You will note below that the two-hour visits include a one-hour wait between consumption of a test breakfast and the second round of measurements. 
While some of this wait period will be used to perform a 24-hour dietary recall (visits 2-5) and give instructions for your assigned diet (visits 2 and 4 ), these activities are not likely to consume the whole 1 hour waiting time. For that reason, it is recommended that you bring homework or some form of activity to occupy yourself between measurements.

Your total compensation for completing the study will be $\$ 80$. You will receive $\$ 20$ following the third lab visit, after completion of the first test diet. You will receive the remaining $\$ 60$ following the fifth and final lab visit, after completing the second test diet and concluding your participation in the study.

- First Visit ( 30min)

○ We will ensure your eligibility for this study by verifying your age and the absence of exclusionary criteria.

- We will explain to you what this study entails and what will be expected of you as a participant in this study.

- After you have had a chance to ask any questions, you will be given a consent form to fill out.

- Upon completing the consent form and agreeing to partake in the study, you will have your body composition measured using the BodPod.

- You will be asked to sit inside the BodPod machine and comfortably rest for 2-5 minutes while your body fat percentage is estimated. This machine is a large eggshaped capsule, with a hatch that has an internal 'panic button' in case you want to open the hatch while you are in there. For this test, we will ask you to bring a bathing suit to wear while you are in the BodPod. This simply minimizes the chances that bulky clothing will affect the reading. We will provide you with a swimming cap to place over your hair; this also minimizes the chances that air caught around your scalp would affect the reading. Your body fat is estimated in this fashion by calculating the amount of air you displace inside the known area of the BodPod. This test should take less than 20 minutes.

- Second Visit ( 3hrs)

$\circ$ You will report to the lab following an overnight (10-hour) fast. 
- Your height, weight, and waist circumference measures will be taken.

- You will be asked a short series of questions regarding your appetite.

- You will have a small amount of blood taken by fingerstick to measure your blood glucose concentration.

- You will have your breath hydrogen measured by exhaling into a small bag.

- You will eat a test breakfast.

- 30 minutes following the breakfast, your blood glucose will be measured again and you will again be asked to answer questions regarding your appetite.

o two hours following the breakfast, your blood glucose and breath hydrogen measurements will be taken again, and you will again be asked to answer questions regarding your appetite.

o During the two hours between finishing the breakfast and the second round of tests, you will be asked to recall what you have had to eat and drink in the past 24 hours.

○ Following the 24-hour recall, you will be given instructions pertaining to the diet to which you have been assigned for the first leg of the experiment. You will also receive reference materials you can take with you to help you adhere to the assigned diet.

You will be asked follow the assigned diet to the best of your ability for three days, beginning after you leave the lab up until 10 hours before the third lab visit. On each of these days you will complete short questionnaires about your appetite, how you feel, and your opinion of the diet. For the 10 hours before the third visit, you will be asked to again refrain from eating or drinking anything except water before reporting to the lab for the third visit.

- Third Visit ( 2hrs)

$\circ$ You will report to the lab following an overnight (10-hour) fast.

- Your height, weight, and waist circumference measures will be taken.

- You will be asked a short series of questions regarding your appetite.

- You will have a small amount of blood taken by fingerstick to measure your blood glucose concentration. 
○ You will have your breath hydrogen measured by exhaling into a small bag.

- You will eat a test breakfast.

- 30 minutes following the breakfast, your blood glucose will be measured again and you will again be asked to answer questions regarding your appetite.

- Two hours following the breakfast, your blood glucose and breath hydrogen measurements will be taken again, and you will again be asked to answer questions regarding your appetite.

- During the two hours between finishing the breakfast and the second round of tests, you will be asked to recall what you have had to eat and drink in the past 24 hours.

Following the third visit, you will receive $\$ 20$ in compensation for completing the first test diet. You may retain this payment even if you choose to withdraw before completion of the study. There will be an 11day period during which you will not be asked to follow any specific diet.

- Fourth Visit ( 2hrs) will include the same procedures as the Second Visit.

You will be asked follow the next assigned diet to the best of your ability for three days, beginning after you leave the lab up until 10 hours before the third lab visit. On each of these days you will complete short questionnaires about your appetite, how you feel, and your opinion of the diet. For the 10 hours before the third visit, you will be asked to again refrain from eating or drinking anything except water before reporting to the lab for the fifth visit.

- Fifth Visit ( 2hrs) will include the same procedures as the Third Visit.

Following the fifth visit, you will have completed your participation in this study. You will receive an additional $\$ 60$ at the end of the fifth visit, for a total of $\$ 80$ in compensation for completion of the study.

Risks or discomfort: 
There are no known risks for the following procedures: questionnaires, consumption the manipulated carbohydrate diets, measures of height, weight, waist circumference, body composition, breath hydrogen, food intake and appetite questionnaires.

The finger prick can result in some slight, short term pain and discomfort. Even though trained, experienced personnel will perform the blood draw using sterile technique, it is possible that minor bruising and infection may occur.

If during the course of the analysis, any incidental findings emerge that indicate a health risk to you (such as high fasting glucose levels), you will be informed and will be advised to consult with your personal physician.

Benefits of this study:

This study will help to determine the effects modified carbohydrate diets on several physiological factors relevant to clinical and therapeutic applications. The direct benefits to you include learning about your body composition, blood glucose, and diet. Upon completing the first test diet you will receive $\$ 20.00$ in compensation. Upon completing the second test diet you will receive $\$ 60.00$ in compensation, for a total of $\$ 80.00$ for completion of the study.

\section{Confidentiality:}

Your participation in this study is confidential. All of your information will be coded by an identification number after all of your data have been collected. None of the results of this study will identify you by name. The document linking your name and identification number and all data collected will be stored on password-protected computers and in locked file cabinets within the locked lab of the investigator. Access will be limited to study investigators. Federal regulations require that data and signed consent documents be kept for three years following completion of the study. The researchers and the University of Rhode Island will protect your privacy, unless they are required by law to report information to city, state or federal authorities, or to give information to a court of law.

Otherwise, none of the information will identify you by name.

In case there is any injury to the subject:

If you have any injury or discomfort as a result of the experiment, you should notify Dr. Kathleen Melanson at (401) 874-4477. You may also 
call the office of the Vice President for Research, 70 Lower College Road, University of Rhode Island, Kingston, Rhode Island, telephone: (401) 874-4328.

Decision to quit at any time:

The decision to take part in this study is up to you. You do not have to participate. If you decide to take part in the study, you may quit at any time. Whatever you decide will in no way penalize you. If you wish to quit, you simply inform Dr. Kathleen Melanson at (401) 874-4477 of your decision.

Rights and Complaints:

This study is part of research being conducted by the University of Rhode Island. If you have any questions or if you are not satisfied with the way this study is performed, you may discuss your complaints with Dr. Kathleen Melanson (401) 874-4477, anonymously, if you choose. In addition, if you have questions about your rights as a research participant, you may contact the office of the Vice President for Research, 70 Lower College Road, Suite 2, University of Rhode Island, Kingston, Rhode Island, telephone: (401) 874-4328.

You have read the Consent Form. Your questions have been answered. Your signature on this form means that you understand the information and you agree to participate in this study.

Signature of Participant

Typed/printed Name

Date
Signature of Researcher

Typed/printed name

Date

Please sign both consent forms, keeping one for yourself.

Would you be willing to be contacted for future studies? If so, sign here: 
Fasting Blood Lipids for sample $n=14($ mean \pm SD)

\begin{tabular}{|c|c|c|c|c|c|c|c|c|}
\hline & \multicolumn{3}{|c|}{$\underline{\text { Low-FODMAP diet }}$} & \multicolumn{3}{|c|}{$\underline{\text { High-FODMAP diet }}$} & \multicolumn{2}{|c|}{$\mathrm{ANOVA}^{1}$} \\
\hline & Baseline & Day 3 & $\Delta$ & Baseline & $\underline{\text { Day } 3}$ & $\Delta$ & p-valu & $\eta^{2}$ \\
\hline Total Cholesterol & $149.5 \pm 24.2$ & $152.8 \pm 28.1$ & 3.3 & $157.9 \pm 25.9$ & $158.7 \pm 28.5$ & 0.80 & .645 & .020 \\
\hline Triglycerides & $86.3 \pm 21.2$ & $76.3 \pm 42.7$ & -10 & $120.9 \pm 49.69$ & $76.6 \pm 19.6$ & $-44.3 * *$ & .065 & .329 \\
\hline HDL & $57.0 \pm 14.0$ & $56.2 \pm 18.7$ & -.80 & $56.9 \pm 11.3$ & $57.8 \pm 13.2$ & 0.90 & .836 & .004 \\
\hline LDL & $75.3 \pm 26.7$ & $77.3 \pm 26.6$ & 2.0 & $74.6 \pm 28.6$ & $83.7 \pm 33.1$ & $9.10 *$ & .174 & .195 \\
\hline
\end{tabular}

Fasting cholesterol response at each time point (mg/dl). Change through each time point was assessed via $2 \times 2$ repeated measures ANOVA.

Analysis via ANOVA did not produce any statistically significant results. Significant within treatment effects were seen during the high FODMAP diet for both triglycerides and LDL cholesterol.

1: data was analyzed via repeated measures ANOVA. $p$-value and $\eta^{2}$ are based on treatment*time interactions.

Within treatment differences

$* *=\mathrm{p}<0.01$

$*=\mathrm{p}<0.05$ 
Fasting Blood lipids $n=8$ compliant with high-FODMAP diet (mean \pm SD)

\begin{tabular}{|c|c|c|c|c|c|c|c|c|}
\hline & \multicolumn{3}{|c|}{$\underline{\text { Low-FODMAP diet }}$} & \multicolumn{3}{|c|}{$\underline{\text { High-FODMAP diet }}$} & \multicolumn{2}{|c|}{ ANOVA $^{1}$} \\
\hline & Baseline & Day 3 & $\Delta$ & Baseline & Day 3 & $\Delta$ & p-value & $\eta^{2}$ \\
\hline Total Cholesterol & $145.2 \pm 20.7$ & $142.4 \pm 24.4$ & -2.8 & $157.7 \pm 31.1$ & $155.4 \pm 36.3$ & -2.3 & .927 & .002 \\
\hline Triglycerides & $78.0 \pm 16.7$ & $63.8 \pm 15.9$ & -14.2 & $130.6 \pm 59.7$ & $78.8 \pm 15.4$ & -51.8 & .083 & .484 \\
\hline HDL & $60 . .0 \pm 9.6$ & $57.0 \pm 10.7$ & -3.0 & $63.8 \pm 7.2$ & $60.8 \pm 12.17$ & -3.0 & .976 & .000 \\
\hline LDL & $69.8 \pm 17.1$ & $71.1 \pm 20.6$ & 1.3 & $70.6 \pm 25.95$ & $82.6 \pm 33.8$ & 12.00 & .133 & .424 \\
\hline
\end{tabular}

Fasting cholesterol response at each time point. Change through each time point was assessed via $2 \times 2$ repeated measures ANOVA. Analysis via ANOVA did not produce any statistically significant results. Significant within treatment effects were seen during the high FODMAP diet for both triglycerides and LDL cholesterol.

1: Indicates use of repeated measures ANOVA. p-value and $\eta^{2}$ represent treatment*time interactions. 


\section{Bibliography}

1. Biesiekierski JR, Rosella O, Rose R, et al. Quantification of fructans, galactooligosacharides and other short-chain carbohydrates in processed grains and cereals. J Hum Nutr Diet. 2011;24(2):154-176.

2. Gibson PR, Shepherd SJ. Evidence-based dietary management of functional gastrointestinal symptoms: The FODMAP approach. J Gastroenterol Hepatol. Feb 2010;25(2):252-258.

3. Barrett JS. Extending Our Knowledge of Fermentable, Short-Chain Carbohydrates for Managing Gastrointestinal Symptoms. Nutr Clin Pract. June 1, 2013 2013;28(3):300-306.

4. Barrett JS, Gearry RB, Muir JG, et al. Dietary poorly absorbed, short-chain carbohydrates increase delivery of water and fermentable substrates to the proximal colon. Aliment Pharmacol Ther. Apr 2010;31(8):874-882.

5. Barrett JS, Shepherd SJ, Gibson PR. Strategies to manage gastrointestinal symptoms complicating enteral feeding. JPEN J Parenter Enteral Nutr. JanFeb 2009;33(1):21-26.

6. Marciani L, Cox EF, Hoad CL, et al. Postprandial changes in small bowel water content in healthy subjects and patients with irritable bowel syndrome. Gastroenterology. Feb 2010;138(2):469-477, 477 e461.

7. Born P. The clinical impact of carbohydrate malabsorption. Arab J Gastroenterol. Mar 2011;12(1):1-4.

8. Fernandez-Banares F, Rosinach M, Esteve M, Forne M, Espinos J, Viver JM. Sugar malabsorption in functional abdominal bloating. Gastroenterology. Apr 2005;128(4):A331-A332.

9. Gibson PR, Barrett JS, Muir JG. Functional bowel symptoms and diet. Intern Med J. Oct 2013;43(10):1067-1074.

10. Brand-Miller JC. Glycemic Load and Chronic Disease. Nutr Rev. 2003;61:S49-S55.

11. Barclay AW, Petocz P, McMillan-Price J, et al. Glycemic index, glycemic load, and chronic disease risk-a meta-analysis of observational studies. Am J Clin Nutr. March 1, 2008 2008;87(3):627-637. 
12. Brighenti F, Benini L, Del Rio D, et al. Colonic fermentation of indigestible carbohydrates contributes to the second-meal effect. Am J Clin Nutr. Apr 2006;83(4):817-822.

13. Wolever TM, Jenkins DJ, Ocana AM, Rao VA, Collier GR. Second-meal effect: low-glycemic-index foods eaten at dinner improve subsequent breakfast glycemic response. Am J Clin Nutr. October 1, 1988 1988;48(4):1041-1047.

14. Liljeberg H, Bjorck I. Effects of a low-glycaemic index spaghetti meal on glucose tolerance and lipaemia at a subsequent meal in healthy subjects. Eur J Clin Nutr. Jan 2000;54(1):24-28.

15. Liljeberg HG, Akerberg AK, Bjorck IM. Effect of the glycemic index and content of indigestible carbohydrates of cereal-based breakfast meals on glucose tolerance at lunch in healthy subjects. Am J Clin Nutr. Apr 1999;69(4):647-655.

16. Nilsson A, Granfeldt Y, Ostman E, Preston T, Bjorck I. Effects of GI and content of indigestible carbohydrates of cereal-based evening meals on glucose tolerance at a subsequent standardised breakfast. Eur J Clin Nutr. Sep 2006;60(9):1092-1099.

17. Cani PD, Knauf C, Iglesias MA, Drucker DJ, Delzenne NM, Burcelin R. Improvement of Glucose Tolerance and Hepatic Insulin Sensitivity by Oligofructose Requires a Functional Glucagon-Like Peptide 1 Receptor. Diabetes. May 1, 2006 2006;55(5):1484-1490.

18. Priebe MG, Wang H, Weening D, Schepers M, Preston T, Vonk RJ. Factors related to colonic fermentation of nondigestible carbohydrates of a previous evening meal increase tissue glucose uptake and moderate glucose-associated inflammation. Am J Clin Nutr. Jan 2010;91(1):90-97.

19. Nilsson AC, Ostman EM, Granfeldt Y, Bjorck IM. Effect of cereal test breakfasts differing in glycemic index and content of indigestible carbohydrates on daylong glucose tolerance in healthy subjects. Am J Clin Nutr. Mar 2008;87(3):645-654.

20. Nilsson AC, Östman EM, Holst JJ, Björck IME. Including Indigestible Carbohydrates in the Evening Meal of Healthy Subjects Improves Glucose Tolerance, Lowers Inflammatory Markers, and Increases Satiety after a Subsequent Standardized Breakfast. J Nutr. April 1, 2008 2008;138(4):732739.

21. Nilsson A, Johansson E, Ekstrom L, Bjorck I. Effects of a Brown Beans Evening Meal on Metabolic Risk Markers and Appetite Regulating Hormones 
at a Subsequent Standardized Breakfast: A Randomized Cross-Over Study. Plos One. Apr 5 2013;8(4).

22. Cani PD, Lecourt E, Dewulf EM, et al. Gut microbiota fermentation of prebiotics increases satietogenic and incretin gut peptide production with consequences for appetite sensation and glucose response after a meal. Am J Clin Nutr. Nov 2009;90(5):1236-1243.

23. Pedersen C, Lefevre S, Peters V, et al. Gut hormone release and appetite regulation in healthy non-obese participants following oligofructose intake. A dose-escalation study. Appetite. Jul 2013;66:44-53.

24. Rosen LA, Ostman EM, Bjorck IM. Effects of cereal breakfasts on postprandial glucose, appetite regulation and voluntary energy intake at a subsequent standardized lunch; focusing on rye products. Nutr J. 2011;10:7.

25. Flint A, Raben A, Blundell JE, Astrup A. Reproducibility, power and validity of visual analogue scales in assessment of appetite sensations in single test meal studies. Int J Obes Relat Metab Disord. Jan 2000;24(1):38-48.

26. Jonnalagadda SS, Mitchell DC, Smiciklas-Wright H, et al. Accuracy of energy intake data estimated by a multiple-pass, 24 -hour dietary recall technique. $J$ Am Diet Assoc. Mar 2000;100(3):303-308; quiz 309-311.

27. Riby JE, Fujisawa T, Kretchmer N. Fructose absorption. Am J Clin Nutr. November 1, 1993 1993;58(5):748S-753S.

28. Grant JD, Bezerra JA, Thompson SH, Lemen RJ, Koldovsky O, Udall JN, Jr. Assessment of lactose absorption by measurement of urinary galactose. Gastroenterology. Oct 1989;97(4):895-899.

29. Simren M, Stotzer PO. Use and abuse of hydrogen breath tests. Gut. Mar 2006;55(3):297-303.

30. Methodology and Indications of H2-Breath Testing in Gastrointestinal Diseases: the Rome Consensus Conference. Aliment Pharmacol Ther. 2009;29:1-49.

31. Lee W, Davidson G, Moore D, Butler R. Analysis of the breath hydrogen test for carbohydrate malabsorption: Validation of a pocket-sized breath test analyser. J Paediatr Child Health. 2000;36(4):340-342.

32. Lohman TG, Roche AF, Martorell R. Anthropometric standardization reference manual. Champaign, IL: Human Kinetics Books; 1988. 
33. Ong DK, Mitchell SB, Barrett JS, et al. Manipulation of dietary short chain carbohydrates alters the pattern of gas production and genesis of symptoms in irritable bowel syndrome. J Gastroenterol Hepatol. Aug 2010;25(8):13661373.

34. Shepherd SJ, Gibson PR. Fructose malabsorption and symptoms of irritable bowel syndrome: guidelines for effective dietary management. J Am Diet Assoc. Oct 2006;106(10):1631-1639.

35. Staudacher HM, Whelan K, Irving PM, Lomer MC. Comparison of symptom response following advice for a diet low in fermentable carbohydrates (FODMAPs) versus standard dietary advice in patients with irritable bowel syndrome. J Hum Nutr Diet. Oct 2011;24(5):487-495.

36. Grubbs F. Procedures for Detecting Outlying Observations in Samples. Technometrics. // 1969;11(1).

37. Cohen J. Statistical Power Analysis. Cur Dir Psychol Sci 1992;1(3):98-101.

38. Darzi J, Frost GS, Robertson MD. Do SCFA have a role in appetite regulation? Proc Nutr Soc. 2011;70(01):119-128.

39. Cani PD, Neyrinck AM, Maton N, Delzenne NM. Oligofructose Promotes Satiety in Rats Fed a High $\square$ Fat Diet: Involvement of Glucagon $\square$ Like Peptide $\square$ 1. Obes Res. 2005;13(6):1000-1007.

40. Baggio LL, Drucker DJ. Biology of incretins: GLP-1 and GIP. Gastroenterology. May 2007;132(6):2131-2157.

41. Stipanuk MH, Caudill MA. Biochemical, Physiological, and Molecular Aspects of Human Nutrition. Elsevier Health Sciences; 2013.

42. Neary MT, Batterham RL. Gut hormones: Implications for the treatment of obesity. Pharmacology \& Therapeutics. 10// 2009;124(1):44-56.

43. Jones HF, Butler RN, Brooks DA. Intestinal fructose transport and malabsorption in humans. Am J Physiol Gastrointest Liver Physiol. Feb 2011;300(2):G202-206.

44. Nanda R, Shu LH, Thomas JR. A FODMAP Diet Update: Craze or Credible? Pract Gastroenterol. 2012:37.

45. Ravich WJ, Bayless TM, Thomas M. Fructose: incomplete intestinal absorption in humans. Gastroenterology. Jan 1983;84(1):26-29. 
46. Eisenmann A, Amann A, Said M, Datta B, Ledochowski M. Implementation and interpretation of hydrogen breath tests. J Breath Res. Dec 2008;2(4):046002.

47. Barrett JS, Gibson PR. Fermentable oligosaccharides, disaccharides, monosaccharides and polyols (FODMAPs) and nonallergic food intolerance: FODMAPs or food chemicals? TherAdv Gastroenterol. 2012;5(4):261-268.

48. Perman JA, Modler S, Barr RG, Rosenthal P. Fasting breath hydrogen concentration: normal values and clinical application. Gastroenterology. Dec 1984;87(6):1358-1363.

49. Flint A, Raben A, Astrup A, Holst JJ. Glucagon-like peptide 1 promotes satiety and suppresses energy intake in humans. J Clin Invest. Feb 1 1998;101(3):515520.

50. Karra E, Batterham RL. The role of gut hormones in the regulation of body weight and energy homeostasis. Mol Cell Endocrinol. 3/25/ 2010;316(2):120128.

51. Barrett JS, Gibson PR. Development and Validation of a Comprehensive Semi-Quantitative Food Frequency Questionnaire that Includes FODMAP Intake and Glycemic Index. J Am Diet Assoc. 10// 2010;110(10):1469-1476.

52. Manning TS, Gibson GR. Prebiotics. Best Practice \& Research Clinical Gastroenterology. 4// 2004;18(2):287-298.

53. Scheppach W, Luehrs H, Menzel T. Beneficial health effects of low-digestible carbohydrate consumption. Br J Nutr. Mar 2001;85 Suppl 1:S23-30.

54. Marcason W. What is the FODMAP diet? J Acad Nutr Diet. Oct 2012;112(10):1696.

55. Cummings JH, Stephen AM. Carbohydrate terminology and classification. Eur J Clin Nutr. Dec 2007;61 Suppl 1:S5-18.

56. Barrett JS, GIbson PR. Clinical ramifications of malabsorption of fructose and other short-chain carbohydrates. Pract Gastroenterolenterology. 2007;31(8):51.

57. Molis C, Flourié B, Ouarne F, et al. Digestion, excretion, and energy value of fructooligosaccharides in healthy humans. Am J Clin Nutr. September 1, 1996 1996;64(3):324-328. 
58. Boler BV, Fahey G, Jr. Prebiotics of Plant and Microbial Origin. In: Callaway TR, Ricke SC, eds. Direct-Fed Microbials and Prebiotics for Animals: Springer New York; 2012:13-26.

59. Torres DP, Gonçalves MdPF, Teixeira JA, Rodrigues LR. Galacto $\square$ Oligosaccharides: Production, Properties, Applications, and Significance as Prebiotics. Comp REv Food Sci Food Saf. 2010;9(5):438-454.

60. Fedewa A, Rao SS. Dietary fructose intolerance, fructan intolerance and FODMAPs. Curr Gastroenterol Rep. Jan 2014;16(1):370.

61. Yao CK, Tan HL, van Langenberg DR, et al. Dietary sorbitol and mannitol: food content and distinct absorption patterns between healthy individuals and patients with irritable bowel syndrome. J Hum Nutr Diet. Aug 32013.

62. Zumbe A, Lee A, Storey D. Polyols in confectionery: the route to sugar-free, reduced sugar and reduced calorie confectionery. Br J Nutr. 2001;85(3):S31.

63. Wong JM, Jenkins DJ. Carbohydrate digestibility and metabolic effects. $J$ Nutr. Nov 2007;137(11 Suppl):2539S-2546S.

64. Swennen K, Courtin CM, Delcour JA. Non-digestible oligosaccharides with prebiotic properties. Crit Rev Food Sci Nutr. 2006;46(6):459-471.

65. Robert K C. The Physiology of the Intestinal Absorption of Sugars. Physiological Effects of Food Carbohydrates. Vol 15: AMERICAN CHEMICAL SOCIETY; 1975:2-19.

66. Barrett JS, Gibson PR. Fermentable oligosaccharides, disaccharides, monosaccharides and polyols (FODMAPs) and nonallergic food intolerance: FODMAPs or food chemicals? Therap Adv Gastroenterol. Jul 2012;5(4):261268.

67. Madsen JL, Linnet J, Rumessen JJ. Effect of nonabsorbed amounts of a fructose-sorbitol mixture on small intestinal transit in healthy volunteers. Dig Dis Sci. Jan 2006;51(1):147-153.

68. Rumessen JJ, Gudmand-Høyer E. Fructans of chicory: intestinal transport and fermentation of different chain lengths and relation to fructose and sorbitol malabsorption. Am J Clin Nutr. August 1, 1998 1998;68(2):357-364.

69. Montalto M, Di Stefano M, Gasbarrini A, Corazza GR. Intestinal gas metabolism. Digestive and Liver Disease Supplements. 2009;3(2):27-29.

70. Braden B. Methods and functions: Breath tests. Best Practice \& Research Clinical Gastroenterology. 6// 2009;23(3):337-352. 
71. Clausen MR, Jorgensen J, Mortensen PB. Comparison of diarrhea induced by ingestion of fructooligosaccharide idolax and disaccharide lactulose (role of osmolarity versus fermentation of malabsorbed carbohydrate). Digestive diseases and sciences. 1998;43(12):2696-2707.

72. Gibson GR. Fibre and effects on probiotics (the prebiotic concept). Clinical Nutrition Supplements. // 2004;1(2):25-31.

73. Lund EK, Johnson IT. Fermentable Carbohydrate Reaching the Colon after Ingestion of Oats in Humans. J Nutr. March 1, 1991 1991;121(3):311-317.

74. Roberfroid MB. Prebiotics: preferential substrates for specific germs? Am J Clin Nutr. February 1, 2001 2001;73(2):406s-409s.

75. Gibson GR, Roberfroid MB. Dietary Modulation of the Human Colonic Microbiota: Introducing the Concept of Prebiotics. J Nutr. June 1, 1995 1995;125(6):1401-1412.

76. Rossi M, Corradini C, Amaretti A, et al. Fermentation of fructooligosaccharides and inulin by bifidobacteria: a comparative study of pure and fecal cultures. Appl Environ Microbiol. Oct 2005;71(10):6150-6158.

77. Bouhnik Y, Raskine L, Simoneau G, et al. The capacity of nondigestible carbohydrates to stimulate fecal bifidobacteria in healthy humans: a doubleblind, randomized, placebo-controlled, parallel-group, dose-response relation study. Am J Clin Nutr. December 1, 2004 2004;80(6):1658-1664.

78. Bouhnik Y, Flourié B, D'Agay-Abensour L, et al. Administration of Transgalacto-Oligosaccharides Increases Fecal Bifidobacteria and Modifies Colonic Fermentation Metabolism in Healthy Humans. J Nutr. March 1, 1997 1997;127(3):444-448.

79. Beards E, Tuohy K, Gibson G. Bacterial, SCFA and gas profiles of a range of food ingredients following in vitro fermentation by human colonic microbiota. Anaerobe. 8// 2010;16(4):420-425.

80. Sarmiento-Rubiano LA, Zúñiga M, Pérez-Martínez G, Yebra MJ. Dietary supplementation with sorbitol results in selective enrichment of lactobacilli in rat intestine. Research in Microbiology. 10// 2007;158(8-9):694-701.

81. Marik PE. Colonic flora, probiotics, obesity and diabetes. Front Endocrinol (Lausanne). 2012;3:87.

82. Macfarlane GT, Macfarlane S. Bacteria, colonic fermentation, and gastrointestinal health. J AOAC Int. Jan-Feb 2012;95(1):50-60. 
83. Tolhurst G, Heffron H, Lam YS, et al. Short-Chain Fatty Acids Stimulate Glucagon-Like Peptide-1 Secretion via the G-Protein-Coupled Receptor FFAR2. Diabetes. February 1, 2012 2012;61(2):364-371.

84. Everard A, Lazarevic V, Derrien M, et al. Responses of gut microbiota and glucose and lipid metabolism to prebiotics in genetic obese and diet-induced leptin-resistant mice. Diabetes. Nov 2011;60(11):2775-2786.

85. Flint A, Raben A, Astrup A, Holst JJ. Glucagon-like peptide 1 promotes satiety and suppresses energy intake in humans. J Clin Invest. Feb 1 1998;101(3):515520.

86. Reimer RA, Pelletier X, Carabin IG, et al. Increased plasma PYY levels following supplementation with the functional fiber PolyGlycopleX in healthy adults. Eur J Nutr. 2010;64(10):1186-1191.

87. Gee JM, Johnson IT. Dietary lactitol fermentation increases circulating peptide YY and glucagon-like peptide-1 in rats and humans. Nutrition. Oct 2005;21(10):1036-1043.

88. Cani PD, Lecourt E, Dewulf EM, et al. Gut microbiota fermentation of prebiotics increases satietogenic and incretin gut peptide production with consequences for appetite sensation and glucose response after a meal. Am J Clin Nutr. Nov 2009;90(5):1236-1243.

89. Connolly ML, Tuohy KM, Lovegrove JA. Wholegrain oat-based cereals have prebiotic potential and low glycaemic index. Br J Nutr. Dec 28 2012;108(12):2198-2206.

90. Östman E. Fermentation as a Means of Optimizing the Glycaemic Index-Food Mechanisms and Metabolic Merits with Emphasis on Lactic Acid in Cereal Products. Lund University; 2003.

91. Jenkins DJ, Wolever TM, Taylor RH, et al. Slow release dietary carbohydrate improves second meal tolerance. Am J Clin Nutr. Jun 1982;35(6):1339-1346.

92. Liljeberg HG, Akerberg AK, Bjorck IM. Effect of the glycemic index and content of indigestible carbohydrates of cereal-based breakfast meals on glucose tolerance at lunch in healthy subjects. Am J Clin Nutr. Apr 1999;69(4):647-655.

93. Nilsson AC, Ostman EM, Granfeldt Y, Bjorck IM. Effect of cereal test breakfasts differing in glycemic index and content of indigestible carbohydrates on daylong glucose tolerance in healthy subjects. Am J Clin Nutr. Mar 2008;87(3):645-654. 
94. Nilsson AC, Ostman EM, Holst JJ, Bjorck IM. Including indigestible carbohydrates in the evening meal of healthy subjects improves glucose tolerance, lowers inflammatory markers, and increases satiety after a subsequent standardized breakfast. J Nutr. Apr 2008;138(4):732-739.

95. Brighenti F, Benini L, Del Rio D, et al. Colonic fermentation of indigestible carbohydrates contributes to the second-meal effect. Am J Clin Nutr. Apr 2006;83(4):817-822.

96. Arumugam V, Lee JS, Nowak JK, et al. A high-glycemic meal pattern elicited increased subjective appetite sensations in overweight and obese women.

Appetite. Mar-May 2008;50(2-3):215-222.

97. Arora T, Loo RL, Anastasovska J, et al. Differential effects of two fermentable carbohydrates on central appetite regulation and body composition. PLoS One. 01/01 2012;7(8):e43263-e43263.

98. Go AS, Mozaffarian D, Roger VL, et al. Heart Disease and Stroke Statistics2013 Update: A Report From the American Heart Association. Circulation. January 1, 2013 2013;127(1):e6-e245.

99. Hardie DG. AMP-activated protein kinase: an energy sensor that regulates all aspects of cell function. Genes Dev. Sep 15 2011;25(18):1895-1908.

100. Hardie DG. AMP-activated protein kinase: a cellular energy sensor with a key role in metabolic disorders and in cancer. Biochem Soc Trans. Jan 2011;39(1):1-13.

101. Motoshima H, Goldstein BJ, Igata M, Araki E. AMPK and cell proliferation AMPK as a therapeutic target for atherosclerosis and cancer. The Journal of physiology. July 1, 2006 2006;574(1):63-71.

102. den Besten G, van Eunen K, Groen AK, Venema K, Reijngoud DJ, Bakker BM. The role of short-chain fatty acids in the interplay between diet, gut microbiota, and host energy metabolism. J Lip Res. Sep 2013;54(9):23252340 .

103. Steinberg GR, Kemp BE. AMPK in Health and Disease. Physiological Reviews. 2009-07-07 19:02:29 2009;89(3):1025-1078.

104. Kawaguchi T, Osatomi K, Yamashita H, Kabashima T, Uyeda K. Mechanism for Fatty Acid "Sparing” Effect on Glucose-induced Transcription: REGULATION OF CARBOHYDRATE-RESPONSIVE ELEMENTBINDING PROTEIN BY AMP-ACTIVATED PROTEIN KINASE. $J$ Biol Chem. February 8, 2002 2002;277(6):3829-3835. 
105. Sakakibara S, Yamauchi T, Oshima Y, Tsukamoto Y, Kadowaki T. Acetic acid activates hepatic AMPK and reduces hyperglycemia in diabetic KK-A(y) mice. Biochem Biophys Res Commun. 6/2/ 2006;344(2):597-604.

106. Fushimi T, Sato Y. Effect of acetic acid feeding on the circadian changes in glycogen and metabolites of glucose and lipid in liver and skeletal muscle of rats. Br J Nutr. Nov 2005;94(5):714-719.

107. Physiological Effects of Food Carbohydrates. Vol 15: AMERICAN CHEMICAL SOCIETY; 1975.

108. Pullinger CR, Eng C, Salen G, et al. Human cholesterol $7 \alpha$-hydroxylase (CYP7A1) deficiency has a hypercholesterolemic phenotype. J Clin Invest. 2002;110(1):109-117.

109. Fushimi T, Suruga K, Oshima Y, Fukiharu M, Tsukamoto Y, Goda T. Dietary acetic acid reduces serum cholesterol and triacylglycerols in rats fed a cholesterol-rich diet. Br J Nutr. May 2006;95(5):916-924.

110. Brighenti F, Casiraghi MC, Canzi E, Ferrari A. Effect of consumption of a ready-to-eat breakfast cereal containing inulin on the intestinal milieu and blood lipids in healthy male volunteers. Eur J Nutr. Sep 1999;53(9):726-733.

111. Queenan KM, Stewart ML, Smith KN, Thomas W, Fulcher RG, Slavin JL. Concentrated oat beta-glucan, a fermentable fiber, lowers serum cholesterol in hypercholesterolemic adults in a randomized controlled trial. Nutr J. 2007;6.

112. Wong JMW, Kendall CWC, de Souza R, et al. The effect on the blood lipid profile of soy foods combined with a prebiotic: a randomized controlled trial. Metabolism. Sep 2010;59(9):1331-1340.

113. Lee CM, Hardy CM. Cocoa feeding and human lactose intolerance. Am J Clin Nutr. May 1, 1989 1989;49(5):840-844.

114. Barrett JS, Irving PM, Shepherd SJ, Muir JG, Gibson PR. Comparison of the prevalence of fructose and lactose malabsorption across chronic intestinal disorders. Aliment Pharmacol Ther. 2009;30(2):165-174.

115. Dale RA, Jensen LH, Krantz MJ. Comparison of Two Point-of-Care Lipid Analyzers for Use in Global Cardiovascular Risk Assessments. Ann Pharmacother. May 1, 2008 2008;42(5):633-639.

116. Parikh $\mathrm{P}$, Mochari $\mathrm{H}$, Mosca L. Clinical utility of a fingerstick technology to identify individuals with abnormal blood lipids and high-sensitivity C-reactive protein levels. Am J Health Promot : AJHP. Mar-Apr 2009;23(4):279-282. 
117. Rowland M, Tozer TN. Clinical Pharmacokinetics and Pharmacodynamics: Concepts and Applications. Wolters Kluwer Health/Lippincott William \& Wilkins; 2011. 